\title{
Assessment of Macropus giganteus as a Biomechanical Model of the Pediatric Thorax
}

\author{
A Thesis \\ Presented to the faculty of \\ The School of Engineering and Applied Science \\ University of Virginia
}

\begin{abstract}
In Partial Fulfillment
of the Requirements for the Degree

Master of Science in Mechanical and Aerospace Engineering
\end{abstract}

by

Sabrina H. Lau

May 2013 
C) Copyright by

Sabrina H. Lau

All rights reserved.

May 2013 


\section{APPROVAL SHEET}

The thesis is submitted in partial fulfillment of the requirements for the degree of Master of Science (Mechanical and Aerospace Engineering)

Sabrina H. Lau, Author The thesis has been read and approved by the examining Committee:

Richard W. Kent, Thesis Advisor

Jeff R. Crandall, Committee Chair

Silvia S. Blemker, Committee Member

Accepted for the School of Engineering and Applied Science:

Dean, School of Engineering and

Applied Science

May 2013 


\section{Acknowledgments}

I would like to express my appreciation for my advisor Dr. Richard Kent for his guidance and advice throughout my graduate studies. I am grateful for his utmost patience and helping me to step away from the trees and look at the forest. I would also like to thank my committee Dr. Jeff Crandall and Dr. Silvia Blemker for their feedback on this work. This work would not have been possible without the financial support of the National Highway and Traffic Safety Administration (Cooperative Agreement No. DTNH22-09-H00247). I would like to thank Mr. Christopher Leigh and Mr. Wesley Fisk from the University of Adelaide in addition to Dr. Sandford Feldman for their assistance in obtaining the kangaroo specimens.

It has been a pleasure working with all of the talented and friendly students and staff at the Center for Applied Biomechanics. Much thanks to Dr. Francisco Lopez-Valdes, and John Lamp for their assistance with these experiments and intellectual chats. Fellow officemates Matthew Kindig and Qi Zhang were a great support and willing to let me pick their brain. Also, thanks to Dr. David Lessley for his help in Vicon processing, and Jim Bolton for his help in designing the test fixtures and then fabricating them.

\section{感謝李醫生的治療, 幫我康復.}

I am truly blessed to have such loving, caring and supportive parents. I have been so inspired by my amazing big brother, Dr. Anthony Lau. I could not have done this without all of his support and encouragement as well as his assistance with making sure my computer functions properly. 


\begin{abstract}
A leading cause of pediatric injury is motor vehicle crashes, in particular injuries to the head which is highly dictated by the thoracic response with the restraint. Due to the dearth of pediatric data available (specifically no existing pediatric thoracic response data in high speed frontal impacts) and the biofidelic uncertainty in current pediatric models, this thesis identifies and assesses an animal model as a surrogate for a six-yearold pediatric thoracic model. The animal model was chosen for its availability and geometric, length, mass and modulus similitude to a human six-year-old. The similitude is important in order to minimize or eliminate the need for scaling. Additionally, the presence of a clavicle was considered necessary for representing belt loading on the human chest. The eastern grey kangaroo satisfied many similitude characteristics and was chosen as the animal model to assess thoracic force-deformation response using three experimental studies: CPR loading, blunt hub loading, and an accelerated sled environment. After juvenile kangaroo carcasses were obtained, CPR and blunt hub loading tests were performed replicating the test conditions of previous pediatric experiments under these loading environments. Finally, kangaroo carcasses were subjected to belted simulated frontal crashes at low $(9 \pm 1 \mathrm{~km} / \mathrm{h})$ and high $(39 \pm 1 \mathrm{~km} / \mathrm{h})$ speeds.
\end{abstract}

In the CPR tests, the kangaroo thorax did not fully recover after each loading cycle which was not as prominent in pediatric data; however, they had a comparable thoracic stiffness response to pediatric subjects. In the blunt hub tests, the juvenile 
kangaroos did not have a large inertial spike in the thoracic force-deformation curve as observed in pediatric subjects. The lack of a sharp inertial force resulted in a third less work done on the chest deformation during loading and unloading of the kangaroo impact compared to pediatric subjects, and consequently more kinetic energy transferred to the kangaroo torso. The thoracic anatomical structures engaged by the loading geometries of the CPR and hub tests differ from a shoulder belt, so the kangaroos were tested in a belted sled accelerated environment. The sled tests showed that positioning the shoulder belt on the kangaroo torso was challenging due to the narrow shoulders and length of the torso. The overall kinematics of the kangaroo sled tests showed large amounts of lumbar lordosis due to the cranial positioning of the belt and more caudal center of gravity, which was probably larger than what a human child would exhibit.

In conclusion, although the eastern grey kangaroo has a similar size and growth development to human children and some anatomical similarities, the thoracic kinematics exhibited by the kangaroos in the sled tests were considered to be unrepresentative of the human child to such an extent that the force-deflection response of the kangaroo would be unlikely to reflect that of the child. This thesis justifies the importance of geometric similitude and mass distribution in the development of pediatric biomechanical models and its effects on thoracic structural behavior when loaded in an inertial environment. 


\section{Table of Contents}

Abstract..

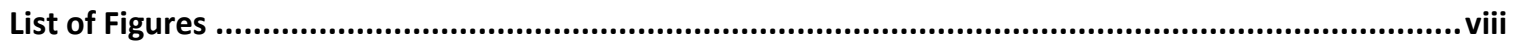

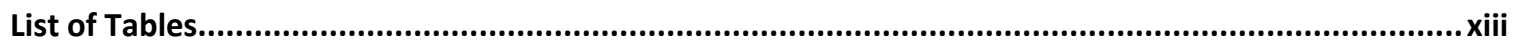

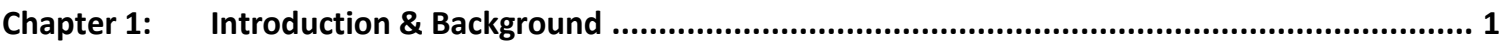

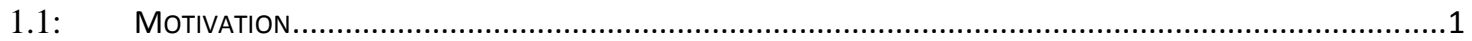

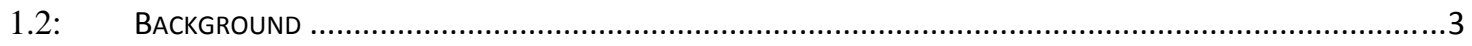

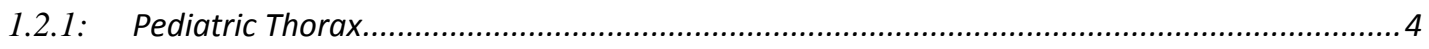

1.2.2: $\quad$ Pediatric PMHS Thoracic Biomechanical Tests .............................................................

1.2.3: $\quad$ Pediatric Volunteer Thoracic Biomechanical Tests ...................................................... 7

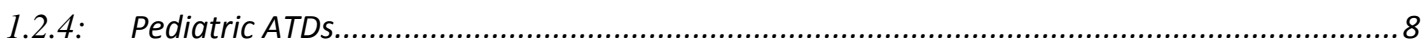

1.2.5: Pediatric Computer Models .................................................................................... 11

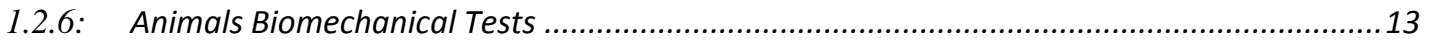

1.2.7: $\quad$ Summary of Limitations of Existing Pediatric Data and Models......................................15

1.3: Justification for Performing a Detailed Biomechanical Assessment of the Kangaroo .................17

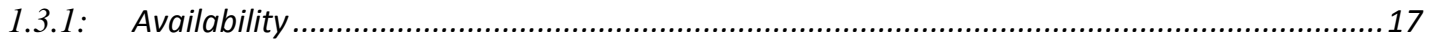

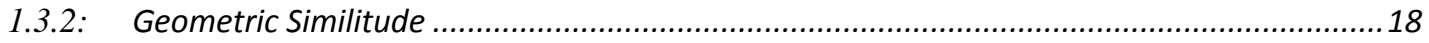

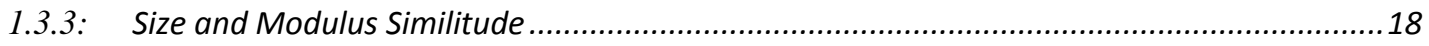

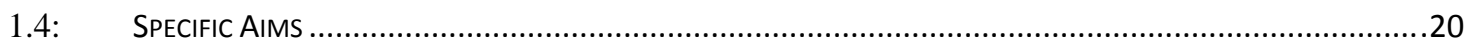

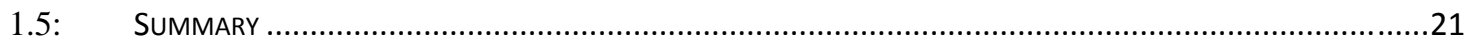

Chapter 2: $\quad$ Kangaroo Subject Acquisition and Anatomy..........................................................22

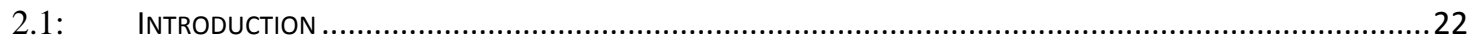

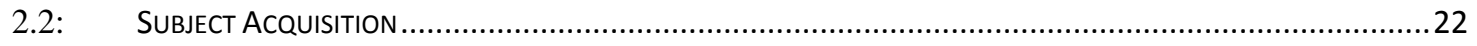

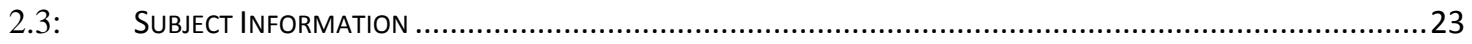




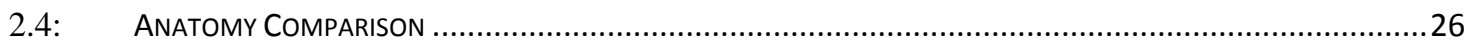

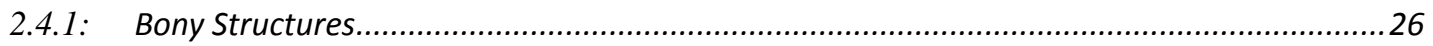

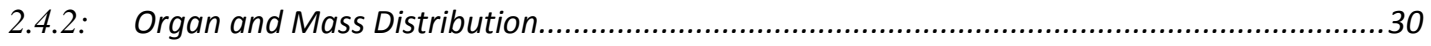

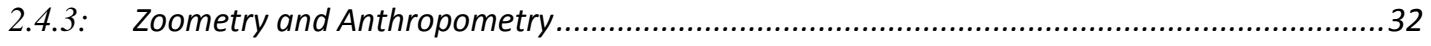

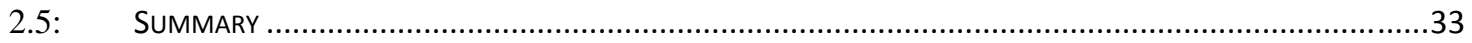

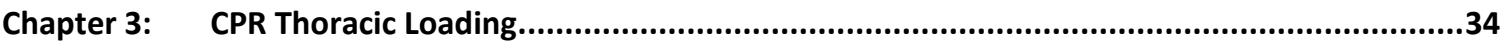

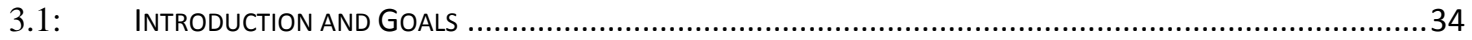

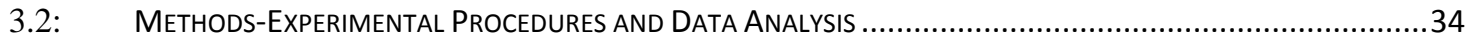

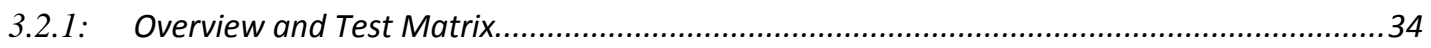

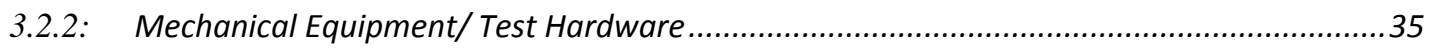

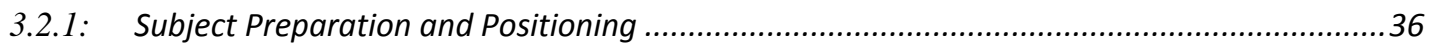

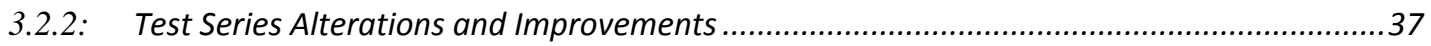

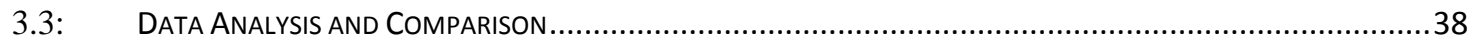

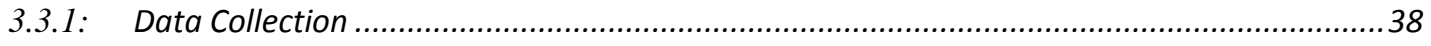

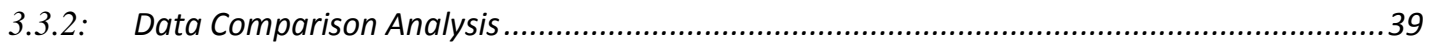

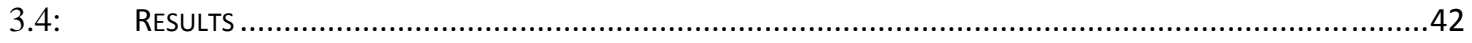

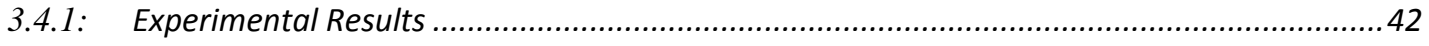

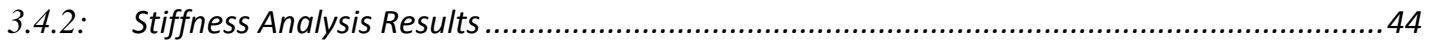

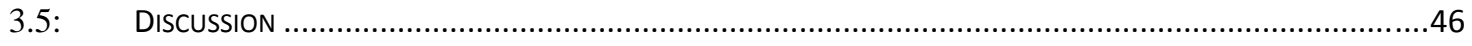

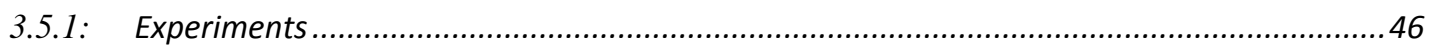

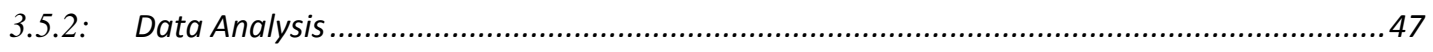

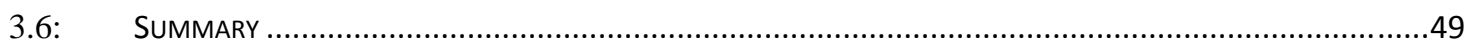

Chapter 4: Hub Impact Loading..............................................................................50

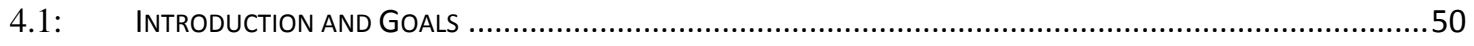

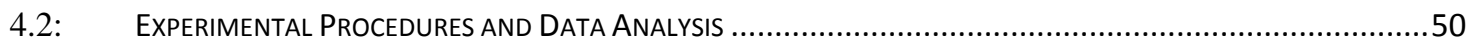




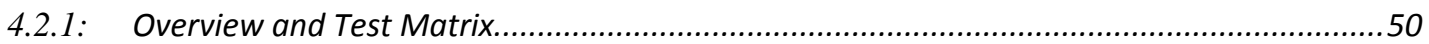

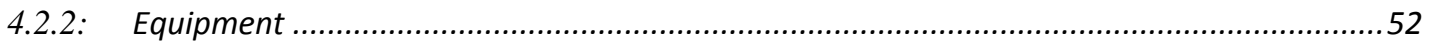

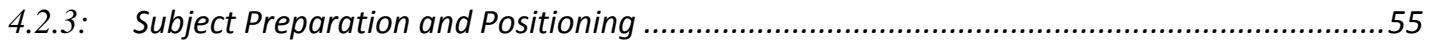

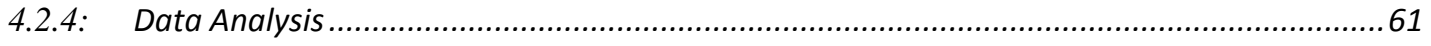

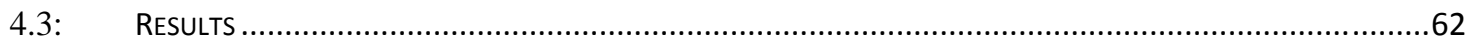

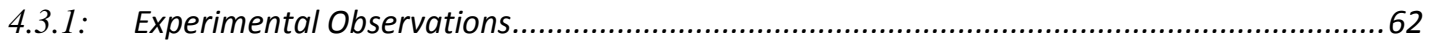

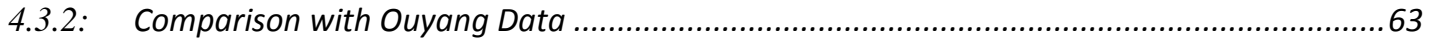

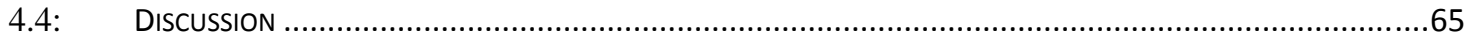

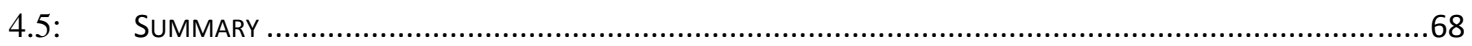

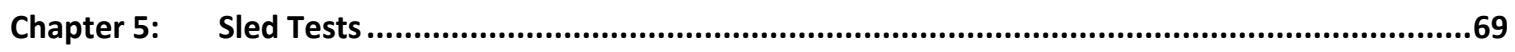

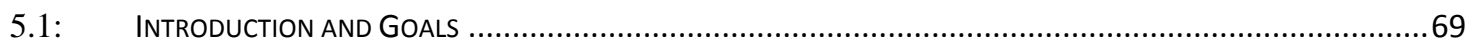

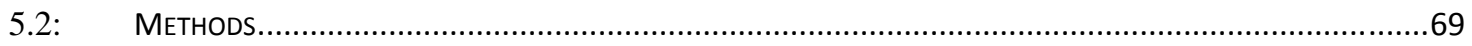

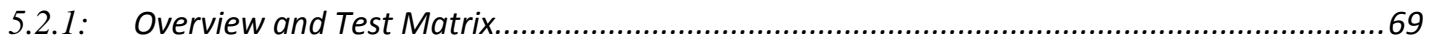

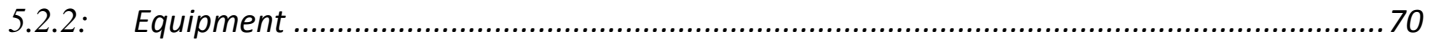

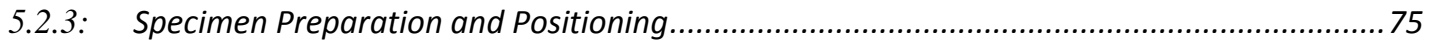

5.2.4: $\quad$ Pilot Subject and Test Methodology Iterations......................................................... 78

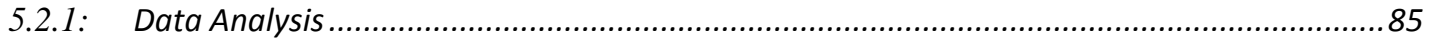

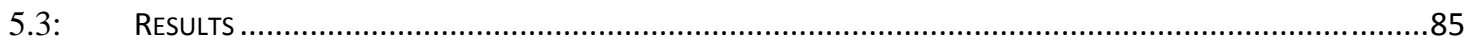

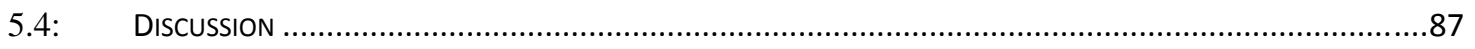

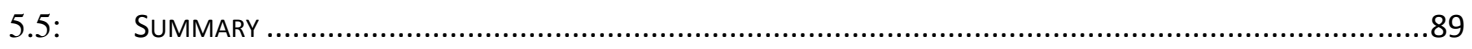

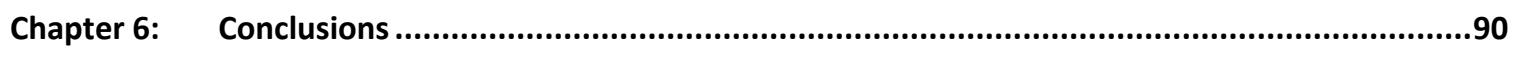

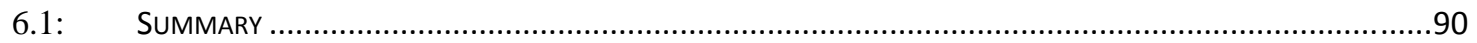

6.2: Strengths of the JuVenile Kangaroo as a Biomechanical Model of the Human 6-Year-Old's Thorax 91 
6.3: limitations of the Juvenile Kangaroo as a Biomechanical Model of the Human 6-Year-old Thorax 92

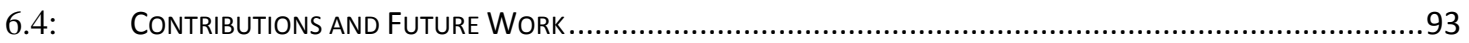

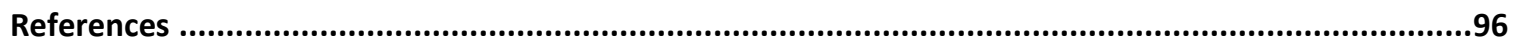

Appendix A: Flexibility of Kangaroo Rib Cage ................................................................................102

Appendix B: CPR Force-deflection Cycles and Thoracic Model Coefficients.........................................103

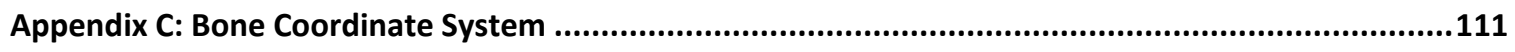

Appendix D: Sled Test Subject Positioning Information ................................................................112

Appendix E: Kangaroo Sled Test Force Deflection Data ................................................................114 


\section{List of Figures}

Figure 1.1 Anterior and oblique view of rib cage with lungs, clavicles, and scapulae ....... 5

Figure 1.2 Thoraces loaded with a hub (left) compared to a diagonal seat belt (right) ... 7

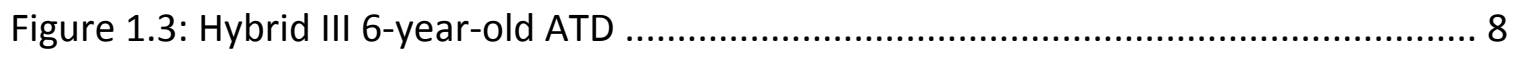

Figure 1.4: Hybrid III-6yo MADYMO ellipsoid model (left) and human child facet model (right) in a blunt frontal impact environment (Source: Parent 2008) ............................ 12

Figure 1.5: Juvenile Eastern Grey Kangaroo standing upright on hind legs .................. 17

Figure 2.1: Correlation between kangaroo whole-body mass (with tail) and human age equivalent based on Snyder et al. (1977). Red arrow indicates mass range of kangaroo subjects. 24

Figure 2.2: Correlation between seated height to the acromion of kangaroo and estimated seated height to acromion based on human age (Snyder et al. 1977). Red arrow indicates seated surface to acromion distance range of kangaroo subjects......... 24

Figure 2.3: Illustration of suprasternal height (61) and gluteal furrow height (75) (Source: Anthrokids) 24

Figure 2.4: Anterior CT images dipicting morphology of a six-year-old human thorax (left) and a juvenile eastern grey kangaroo thorax (right) with the right clavicles highligthed (images not to scale). 27

Figure 2.5: Right saggital CT images depicting morphology of a six-year-old human thorax (left) and a juvenile eastern grey kangaroo thorax (right) (images not to scale). 28 
Figure 2.6: Posterior CT images depicting morphology of a six-year-old human thorax (left) and a juvenile eastern grey kangaroo thorax (right) showing the number of floating ribs (images not to scale). 28

Figure 2.7: Superior CT images depicting morphology of a six-year-old human thorax (left) and a juvenile eastern grey kangaroo thorax (right) with the scapulae and right clavicles highlighted (images not to scale). 29

Figure 2.8: Anterior CT images depicting thoracic and lumbar spine morphology of a human six-year-old (left) and a juvenile eastern grey kangaroo (right) (images not to scale). 30

Figure 2.9: Human 6-year-old and juvenile organ mass comparison. 31

Figure 2.10: CT frontal cross-section of human 6-year-old (left) and juvenile kangaroo (right) showing distribution of internal organs. Red outline of lower thoracic organs and intestinal tract (images not to scale) 31

Figure 2.11: Thoracic breadth measurements of juvenile kangaroo subjects and human six-year-olds. 32

Figure 2.12: Thoracic circumference measurements of juvenile kangaroo subjects and human six-year-olds 33

Figure 3.1: CPR Thumper device (left) and force-deflection sensor (FDS puck) (right).... 36

Figure 3.2: CPR FDS puck configuration for hub test subjects (left) and sled test subjects (right) 37 
Figure 3.3: Re-orienting FDS puck for subject K06F due to the concavity of the sternum

Figure 3.4: Lumped mass model of the chest used for CPR analysis. F is the time-history of the force applied to the chest during CPR, $k$ is the elastic coefficient of the chest, $\mu$ is the viscous coefficient of the chest, $m_{s}$ is the mass of the sternum and $x_{s}$ is the displacement time-history of the sternum. 40

Figure 3.5: Individual force-deflection curves for kangaroo subjects compared to pediatric 6yo and 7yo PMHS (top), and plots with cycles zeroed with respect to deflection (bottom) 43

Figure 3.6: Elastic force at $10 \%$ of chest depth for juvenile kangaroo and pediatric PMHS CPR loading tests 44

Figure 3.7: Average stiffness, $k$, (calculated using Equation 3.2) per cycle for juvenile kangaroo carcasses and pediatric PMHS CPR tests. 45

Figure 3.8: Pediatric (left) and kangaroo (right) CPR force-deflection curves plotted on top of max deflection points and elastic force curve from model for 8-year-old patient.

Figure 3.9: Pediatric (left) and kangaroo (right) CPR force-deflection curves plotted on top of max deflection points and elastic force curve from model for 9-year-old patient.

Figure 4.1: Outline of hub impact locations (red outline-T4 impact, green outline-XP impact) on CT of kangaroo thorax (KO8M) 51 
Figure 4.2: Schematic of kangaroo hub test set-up................................................. 53

Figure 4.3: Details of transfer piston with impacting hub surface ................................ 55

Figure 4.4: Spinal mounting method-Wood screws (A) were screwed into the lateral spinous processes of the target vertebra with the objective being to maximize the cortical bone through which it passed. A two piece radio-translucent plastic assembly (B) clamped to the heads of the screws forming the base for the accelerometer and marker plates (C) CT image of the mounts rigidly attached to vertebral bodies and pelvis.

Figure 4.5: Vicon marker plate arrays and single marker locations on kangaroo hub test subject. 57

Figure 4.6: Sagittal view of T4 impact level (left) and XP impact level (right) on subject K10M positioned on arm supports 59

Figure 4.7: Hub Impact Test Hardware with labels defined in Table 4.4 60 Figure 4.8: Ouyang old cohort (average age $=7.3$ years) compared to kangaroo forcedeflection curves. 64

Figure 4.9: Ouyang young cohort (average age $=2.6$ years) compared to kangaroo forcedeflection curves. 64

Figure 4.10: Kangaroo T4 level impact force-deflection curves compared to the corridor from data reanalyzed by Parent et al. (2010). 65 Figure 5.1: Custom designed adjustable pelvis restraint positioned on sled buck seat (left) with parts highlighted in sled set-up (right). 72 
Figure 5.2: CT images of KO2F pelvic region bony and soft tissue (top) and CT images of

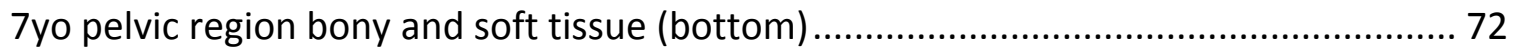

Figure 5.3: Sled test hardware. Photos of pre-test 1440 with subject K05M and 1436

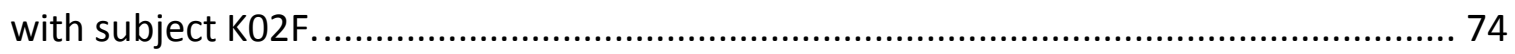

Figure 5.4: Vicon marker plates and single marker locations for K02F and K05M (head) with descriptions in Table 5.4 77

Figure 5.5: Screen shots of trial subject K01F test 1433 showing torso rotation and belt

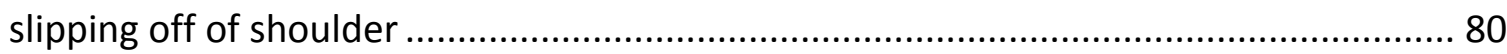

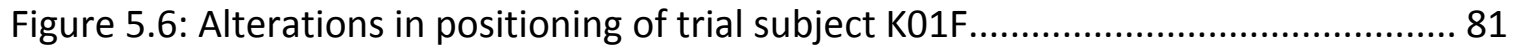

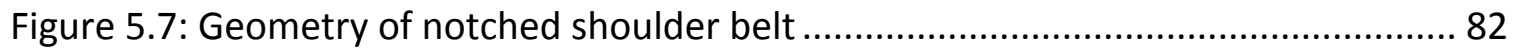

Figure 5.8: Shoulder belt load limiter with indicated score lines and safety tether circled in red. 84

Figure 5.9: Transition from pelvis block strap to pelvis block L-bracket 85 Figure 5.10: Upper shoulder belt force time history for low speed (left) and high speed (right) sled tests 86

Figure 5.11: Shoulder belt positioned on K02F, K05M, K06F, and human child (from left to right) Sternum outlined with location of xiphoid process indicated by red circle and location of belt crossing sternum indicated by red arrows.......................................... 86

Figure 5.12: Motion of K05M during high speed test............................................... 87 


\section{List of Tables}

Table 1.1: Limitations of Pediatric PMHS and Volunteer Tests 16

Table 2.1: Summary of all kangaroo specimen information and anatomical

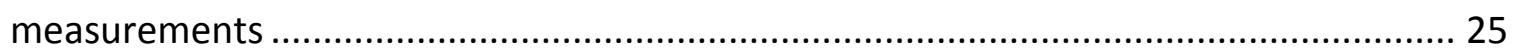

Table 2.2: Number of vertebrae for humans and kangaroos .................................... 30

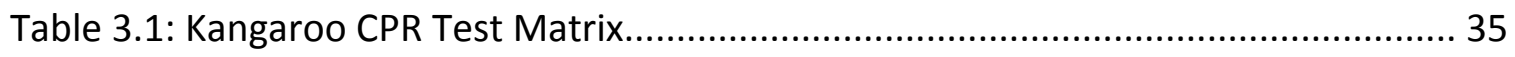

Table 3.2: Pediatric PMHS CPR Test Matrix (Kent et al. 2012) ..................................... 40

Table 3.3: Kangaroo subject's sternum angle in CPR tests ...................................... 43

Table 4.1: Kangaroo Hub Test Matrix and depths at impact levels ............................... 51

Table 4.2: Pediatric PMHS subjects used in hub tests by Ouyang et al. (2006) ............... 52

Table 4.3: Equipment Specifications-Hub Impact System .......................................... 54

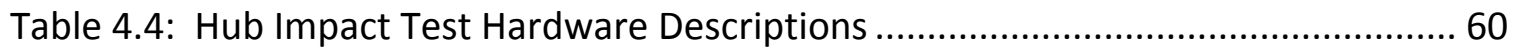

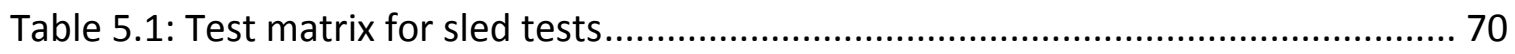

Table 5.2: Equipment Specifications-Sled System ..................................................... 73

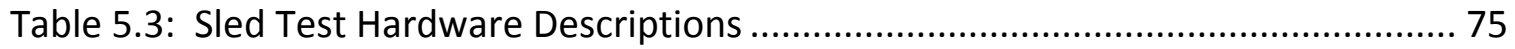

Table 5.4: Vicon marker and array abbreviations and locations ................................. 77

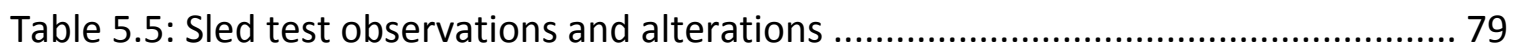

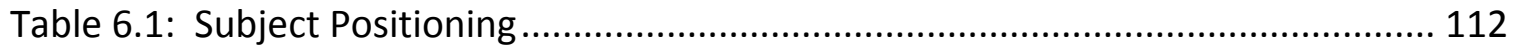

Table 6.2: Additional Positioning Measurements................................................. 113 


\section{Chapter 1: Introduction \& Background}

\section{1: Motivation}

Motor vehicle crashes are the leading cause of pediatric injury-related deaths in the United States (CDC 2007). In 2009, 1314 children under the age of 14 were killed in motor vehicle crashes and approximately 179,000 were injured (NHTSA 2009, CDC 2011). To help minimize fatality rates, the Federal Motor Vehicle Safety Standard (FMVSS) No. 213 specifies performance requirements for child restraint systems (CRS) by testing CRS designs by using anthropomorphic test devices (ATDs) or crash test dummies.

Ideally, the ATD should be biofidelic, where it behaves similarly to a living human under comparable loading conditions. The current Hybrid III 6-year-old ATD was developed based on scaling adult data (Irwin and Mertz 1997). The scaling assumptions used, based on size, mass, and material properties may not accurately predict the child thoracic response (Kent et al. 2009, 2012), due to structural differences, body proportions, and biomechanical tissue properties (Burdi et al. 1969, Franklyn et al. 2007). Previous studies show trends in thoracic stiffness which increases from childhood to middle age, and then decreases as adults age (Maltese et al. 2008, Kent et al. 2009, 2012). The scaling paradigms used previously do not predict the increasing and decreasing stiffness phenomenon. Computational models of the pediatric thorax, used in the development of ATDs, are also based on scaling and therefore also not sufficiently validated (Forbes et al. 2008). 
Pragmatically, it is difficult to obtain pediatric biological material for impact biomechanics. As a result, pediatric biomechanical impact response data are limited, particularly the 6-year-old thoracic response in high speed frontal impacts, leading to challenges in developing biofidelic child dummies and computer models. The need for data that apply to the child without scaling motivated this research, which is focused on assessing an animal biomechanical model.

The 6-year-old age is of particular interest since there is an ATD for this age and this is the age when children transition from harness-type CRS to booster seats, which employ the adult lap and diagonal shoulder belts. The design of safety restraints is such that the shoulder belt is the main load path restraining the thorax. The restraint system should be designed to modulate the force and deformation response of the chest. Forces are applied to the thorax from belt tension, which results in the chest deforming. This deformation involves complex three-dimensional motion (Shaw et al. 2009), but a simple descriptor is the posterior displacement of the sternum relative to the thoracic spine, which is the thoracic measurement used in ATDs. This displacement is often referred to as chest deflection, and will be used to describe this phenomenon throughout this thesis.

The deformation of the chest is important because it predicts injury and dictates head trajectory (Kent et al. 2009). Children have very compliant rib cages and rarely sustain rib fractures (Inan et al. 2007); however, head injuries are the most common serious injuries sustained by children in motor vehicle crashes (Arbogast et al. 2002, 2004, Levy 1972, Sherwood et al. 2003a). Factors influencing head trajectory have been 
studied, including cervical spinal kinematics (Arbogast et al. 2009, Luck et al. 2008) and thoracic spinal kinematics (Lopez-Valdes et al. 2010). This study focuses on a relatively understudied factor: the relationship between applied belt loads and the resulting chest deformation.

Though deformation of the chest is related to injury, this thesis focuses purely on the structural behavior of the chest and not necessarily its consequences for thoracic injury. From the standpoint of designing more biofidelic 6-year-old ATDs and computational models, a design specification based on a realistic loading profile of the overall thoracic response at potentially injurious frontal decelerations will be an important contribution to the pediatric injury biomechanics field.

\section{2: Background}

Human surrogates have been used extensively in injury biomechanics research. Five common surrogates used in the field of injury biomechanics are cadavers or post mortem human surrogates (PMHS), volunteers, anthropometric test devices (ATDs), computational models, and animals. Each of these surrogates has advantages and disadvantages regarding its merit in relation to human anthropometry, human anatomy, physiologic response, its ability to test at injurious levels, and its usefulness to predict injury (Crandall et al. 2011). To design biofidelic computational models and ATDs, reliable pediatric thoracic response data is needed. A review of the literature is presented below to motivate and provide context for the work to be described in this thesis. The review starts with a description of the relevant anatomical and 
biomechanical characteristics of the pediatric thorax. The tools that are used to model these characteristics for the design of crash protection systems are then discussed.

\subsection{1: $\quad$ Pediatric Thorax}

The main bony structure of the thorax is the rib cage (Figure 1.1) composed of twelve pairs of ribs connected posteriorly to twelve thoracic vertebrae. Anteriorly, the first seven pairs of ribs connect directly to the sternum by a flexible linkage, i.e. costal cartilage. Ribs 8-10 are false ribs that attach to the cartilaginous portion of the adjacent superior rib and do not directly connect to the sternum. Ribs 11 and 12 are floating ribs that do not attach anteriorly.

The sternum consists of six bones; the superior bone is called the manubrium. The gladiolus or body of the sternum is comprised of four sternabrae, where the sternabrae fuse between the ages of puberty and twenty-five years (Gray 1918, Scheuer and Black 2000). The inferior bone of the sternum is a mostly cartilaginous piece called the xiphoid process. The clavicles and scapula are part of the shoulder joint, where the clavicles articulate with the manubrium connecting the upper extremities to the thorax. The sternum shifts inferiorly from birth to adulthood such that the rib angle is more horizontal in pediatrics compared to adults (Scheuer and Black 2000).

The rib cage in children is more flexible than adults as most cartilaginous portions of the bone have not ossified (Franklyn et al. 2007). The slightly conical rib cage encloses vital internal organs of the respiratory and cardiovascular systems including the heart, and lungs. The posterior inferior half of the thoracic wall protects abdominal organs, e.g. stomach, liver, spleen and kidneys. The child's torso is 
proportionately larger than the rest of the body compared to adults; therefore, the center of gravity of the child is situated higher than in adults (Sturtz 1980).

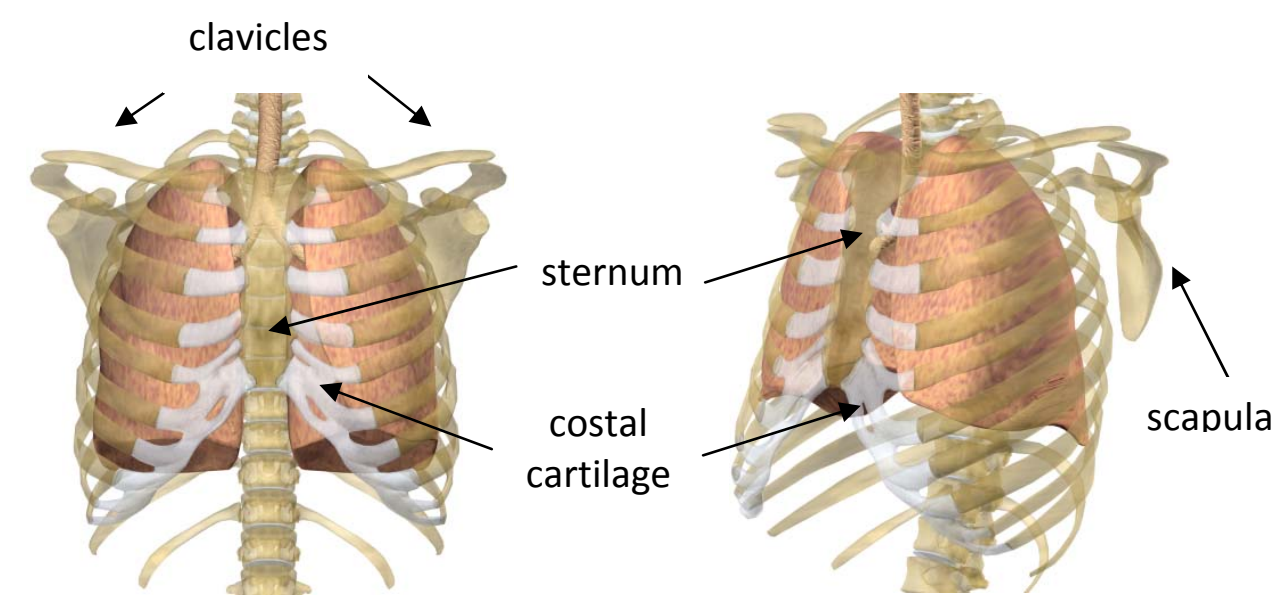

Figure 1.1 Anterior and oblique view of rib cage with lungs, clavicles, and scapulae

\subsection{2: $\quad$ Pediatric PMHS Thoracic Biomechanical Tests}

Thoracic biomechanical studies on pediatric PMHS are scarce. Loading conditions performed on pediatric PMHS include blunt hub loading, cyclic cardiopulmonary resuscitation (CPR) loading, stationary belt loading, and sled tests with various restraint systems. Hub impact tests were performed on nine pediatric PMHS 212 years of age (Ouyang et al. 2006). This pediatric hub impact data were re-analyzed by Parent et al. (2010) minimizing instrumentation and processing error to improve the fidelity of the data. Sled tests were performed on twelve pediatric PMHS ages 2-13 years old (Kallieris et al. 1976 and 1978, Mattern et al. 2002, Wismans et al. 1979, Dejeammes et al. 1984, Brun-Cassan et al. 1993). Restraints used for these pediatric PMHS tests included a lap belt with a shield booster, 4-point and 5-point harnesses, and 3-point belts. Obtaining pediatric PMHS subjects is difficult, but recently, Kent et al. (2009, 2011, 2012) procured three pediatric PMHS, ages 6, 7 and 15 years old. These 
subjects were restricted to non-impact testing so these studies performed cyclic CPR and diagonal-belt table-top tests on the pediatric PMHS, measuring thoracic forcedeflection response. The CPR tests collected data from non-injurious cyclic compressions using a force-deflection sensor (FDS puck) positioned along the sternum midline. In the table-top tests, the thorax is positioned supine on a flat surface and loaded anteriorly with a shoulder belt at sternal displacement rates comparable to those observed in a sled tests. The chest deflection, posterior reaction forces and applied belt forces were measured while the subject was stationary.

In addition to the scarcity of the existing pediatric PMHS thoracic biomechanics tests, there are limitations to these studies in terms of understanding the thoracic forcedeflection response from a shoulder belt in potentially injurious frontal loading. Hub impacts, centered mid-sternum engage different thoracic structures than a shoulder belt (Figure 1.2), and a properly positioned belt does not involve an impact, such that the relative velocity between the belt and the contacting torso is zero (Kent et al. 2004). Three of the sled tests (subject ages 12 and 13 years old) used a three-point belt restraint system, but these sled tests were performed in the 1970's when instrumentation technology was limited, so a chest deformation measurement was not recorded. The CPR tests had similar loading geometry loading concerns as the hub tests since the FDS puck contacted mainly the sternum, which is unrepresentative of anatomical structures engaged by a shoulder belt (Figure 1.2). Additionally, the rate of sternal displacement in the CPR tests is an order of magnitude lower than crash rates. The well defined boundary conditions of the table-top tests differ from the dynamic 
inertial loads of sled tests. The relationship between applied belt force and the resulting thoracic deformation may be different in the sled environment than in the table-top environment for the following reasons: transient belt angles, inertial loads, and free posterior movement compared to a fixed back. The differences between these two environments and the consequences for thoracic force-deformation response are not fully understood.
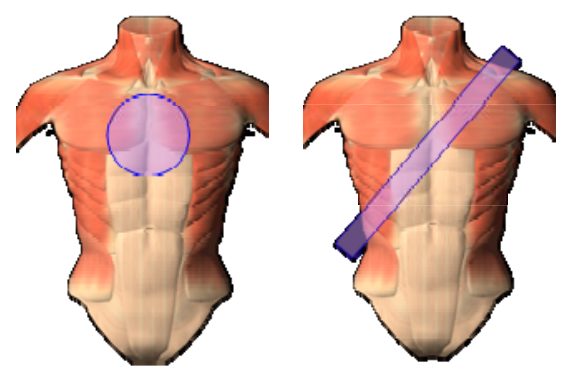

Figure 1.2 Thoraces loaded with a hub (left) compared to a diagonal seat belt (right).

\subsection{3: $\quad$ Pediatric Volunteer Thoracic Biomechanical Tests}

Human pediatric volunteers are identical to the subject of interest and provide physiological responses unlike PMHS. Maltese et al. (2008\}) recorded cyclic forcedeflection measurements clinically during CPR on eighteen patients, age 8 to 22 years. Arbogast et al. (2009\}) performed low-speed (deceleration pulse $<4.5 \mathrm{~g}$ ) non-injurious sled tests on 20 male pediatric volunteers (6-14 years old) which focused on subject spinal kinematics and chest deflection data were not reported.

The test conditions of the clinical CPR patients have the same limitations as the pediatric PMHS CPR tests described in the previous sub-section (1.2.2:). The CPR tests from Kent et al. $(2009,2012)$ were based on the loading protocol from Maltese et al (2008). Considering both sets of pediatric CPR data along with existing adult CPR data, 
Maltese et al. $(2008\})$ and Kent et al. $(2009,2012)$ show that thoracic stiffness increases through mid-life and then decreases in the later years of life. This indicates there may not be a linear relationship between pediatric and adult thoracic stiffness. Volunteer tests are restricted to non-injurious loading regimes, so there is still a void to fill in understanding the pediatric response in potentially injurious loading conditions.

\subsection{4: $\quad$ Pediatric ATDs}

ATDs can be tested at injurious loading regimes unlike volunteer subjects. Families of ATDs have been developed with dummies ranging from 6 months old to adults. The child ATDs developed in the United States starting in 1987 include the CRABI (Child Restraint Air Bag Interaction) dummies (ages 6, 12 and 18 months old) and the Hybrid III (HIII) dummies (ages 3, 6, and 10 years old). The adult HIII ATDs include the $5^{\text {th }}$ percentile female, and the $50^{\text {th }}$ and $95^{\text {th }}$ percentile male. The European designed $\mathrm{Q}$ series child ATDs, which were originally the P series, include the Q0, Q1, Q1.5, Q3, Q3s, Q6, Q6s, and Q10, where the numbers indicate the age in years and the " $\mathrm{s}$ " indicates ATDs designed for side impacts. The Q3 was the first Q-dummy developed in 1996 and the Q6 was developed later based on the Q3.

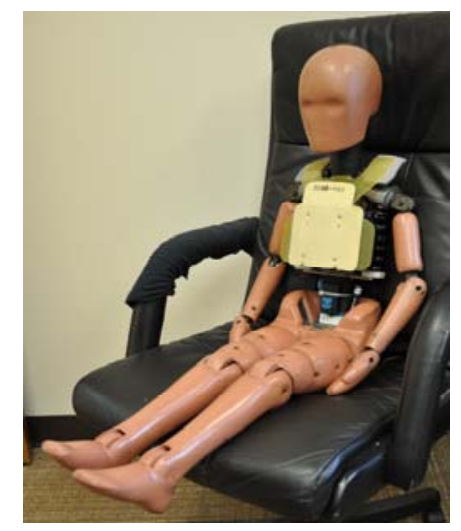

Figure 1.3: Hybrid III 6-year-old ATD 
The development of the HIII 6-year-old (HIII-6yo) biofidelity requirements is based on scaling from the adult $\mathrm{HIII} 50^{\text {th }}$ percentile male biofidelity requirements (Irwin and Mertz 1997). Scaling is based on dimensional analysis, and the three fundamental dimensionless ratios for the structural analysis used in the biomechanics field include a length scale ratio, mass density ratio, and modulus of elasticity ratio shown below (Eppinger et al. 1999).

$$
\begin{array}{ll}
\text { Length Scale Ratio: } & \lambda_{\mathrm{L}}=\mathrm{L}_{1} / \mathrm{L}_{2} \\
\text { Mass Density Ratio: } & \lambda_{\rho}=\rho_{1} / \rho_{2} \\
\text { Modulus of Elasticity Ratio: } & \lambda_{\mathrm{E}}=\mathrm{E}_{1} / \mathrm{E}_{2}
\end{array}
$$

Dimensional scaling involves assumptions of geometric similitude and loading of homologous structures; however, Franklyn et al. (2007) shows differences in mass distribution, and material properties between children and adults. To account for these differences, the study by Irwin and Mertz (1997) calculated scale factors for the HIII-6yo based on characteristic dimensions and segment masses of the head, neck, torso, thigh, and leg, as well as the elastic modulus of cranial bone. The validity of this scaling methodology for material properties is uncertain, since there is not a monotonically increasing stiffness change with increasing mass, modulus, and length (Maltese et al 2008, Kent et al 2012). Additionally, the scaling for the elastic modulus is limited to one tissue type (e.g. bone) and does not take into account the effect of hard and soft tissues which comprise the thorax (Ash et al. 2009).

In addition to uncertainty in modulus scaling, design differences between the Q6 and HIII-6yo ATDs include different structures, anthropometry and weight distribution. 
The size and body weight of the HIII-6yo is based on anthropometric studies of 6-yearold children in the United States. The Q6 anthropometric data is based on the Child Anthropometry Database (CANDAT) which is child data from different regions, i.e. US, Europe and Japan. Mass distribution differences between the HIII-6yo and the Q6 include greater torso mass of the HIII-6yo, but greater mass of the extremities in the Q6, in particular the lower extremities (Hammarstrom and Jorlov 2011). Differences in thoracic measurement consist of a taller sitting height of the HIII-6yo, but wider shoulders for the Q6 (Hammarstrom and Jorlov 2011). The Q6 has a one piece rib cage with an angled sternum, whereas the HIII-6yo has six horizontal ribs with a sternum parallel to the spine. Also, the Q6 has distinct clavicle elements and the HIII-6yo does not.

To understand the differences in the two 6yo ATDs, studies have been performed to compare the responses of the two 6-year-old ATDs as well as the ATDs response compared to human volunteer data. Lubbe (2010) compared the HIII-6yo and the Q6 at $64 \mathrm{~km} / \mathrm{h}$ frontal impact tests and found significant differences in chest deflection for comparable belt loads. This difference was likely due to the seatbelt sliding towards the neck from the angled thorax of the Q6. Seacrist et al. (2010) compared the HIII-6yo to pediatric human volunteers at non-injurious low speed tests and reported reaction forces and head and spinal kinematics. Seacrist et al. (2012) expanded the aforementioned study to include the HIII-10yo and the Q6 and Q10 dummies for comparison of spinal curvature and reaction forces. This study found that both 6-year-old ATDs had significantly greater shoulder and lap belt loads than the 
pediatric volunteers, but the seat pan shear of the pediatric volunteers was larger compared to the ATDs. Both 6-year-old ATDs reported decreased spinal excursions, which is probably a result of the stiff spinal structures. The stiff spines of child and adult ATDs were also found to be unrepresentative of humans in several other studies (Ash et al. 2009, Sherwood et al. 2003b, and Shaw et al. 2001).

The ATD comparison studies show little consensus between the 6-year-old ATD response, and differences between the ATDs and human volunteer and PMHS tests. The differences in chest deflection, reaction loads, and spinal kinematics reflect the lack of knowledge of biofidelic response of the pediatric thorax. The limited amount of pediatric data led to scaling assumptions used to develop the ATDs, but there is uncertainty in scaling assumptions. There are also design tradeoffs that need to be considered for ATDs; they need to be biofidelic but also rugged enough to withstand repeated testing and provide repeatable responses for given loads.

\subsection{5: $\quad$ Pediatric Computer Models}

Computational models of the human six-year old have been developed recently to aid in the development of ATDs. Multi-body numerical models developed in MADYMO (Mathematical Dynamic Models) by TNO (Netherlands) include ellipsoid models of the HIII-6yo (Figure 1.4, left) and Q6 ATDs and a child human facet model (Figure 1.4, right) (Forbes 2008). The ellipsoid ATD models were developed based on their respective physical ATDs. The 6-year-old human facet model was developed by scaling the extensively validated $50^{\text {th }}$ percentile male model to a 6 -year-old child. Anthropometric scaling was based on the CANDAT database, and other model 
parameters scaled include mass, moments of inertia, joint characteristics, ellipsoids and penetration characteristics, force models, and sensor locations. The model was also scaled using the biofidelity response corridors from Irwin and Mertz (1997).
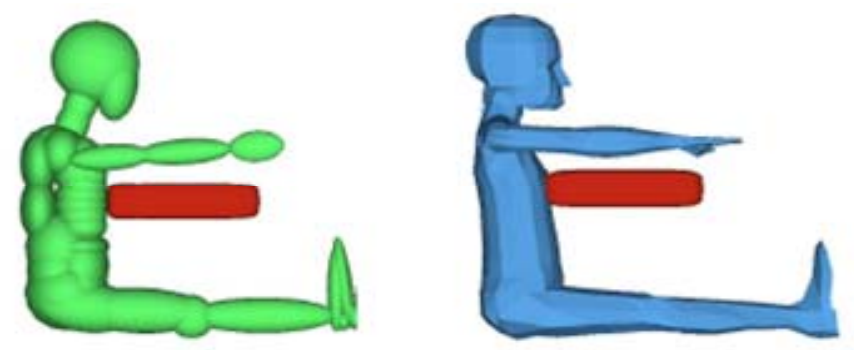

Figure 1.4: Hybrid III-6yo MADYMO ellipsoid model (left) and human child facet model (right) in a blunt frontal impact environment (Source: Parent 2008)

To optimize characteristics of the computer models, they were validated against relevant impact responses. The blunt hub impact tests by Ouyang et al. (2006) were one set of validation tests for the human child facet model. Laboratory sled tests (replicating Kallieris et al. 1976, 1978) were run with physical HIII-6yo and Q6 ATDs with a shield booster restraint and 3-point belt restraint. Facet models of the sled test environment and restraints were validated against the laboratory tests using the ATD elliptical models. The validated sled test environment was then used with the human child facet model for validation against the pediatric PMHS (ages 6, 12, and 13 years old) performed by Kallieris et al. $(1976,1978)$ using either the shield booster restraint (6 year old) or 3-point-belt restraint (12 and 13 year olds) as used in the original laboratory tests. The 6-year-old facet model was scaled using only anthropometry to match the 12 and 13 year old PMHS subjects. Overall the computer model simulations showed similar trends to laboratory experiments. 
Pediatric computational models developed through scaling and experimental validation share the same limitations as ATDs which is the lack of biofidelic data. Validation to the hub impacts provides the necessary response for the given loading condition, but it is insufficient to characterize the thoracic response under a non-impact diagonal belt load. The human child model validated to sled tests has the same limitations as the experimental sled tests by Kallieris et al. $(1976,1978)$, i.e. only the 12 and 13- year-old subjects used a 3-point-belt and chest deflection was not measured in those tests.

\subsection{6: $\quad$ Animals Biomechanical Tests}

Animals have been used as pediatric surrogates in biomechanical tests. An advantage of animal models is the ability to test a biological model with physiological response under potentially injurious loadings, and they are a frangible model compared to ATDs. Particular species were found to be useful surrogates for humans because of focused anatomical similarities; however, there are limitations when considering whole animal subjects. Important factors to consider in animal models are anatomical similarities, including structural anatomy, material properties, and organ positioning. Animal surrogates used previously in biomechanics test include porcine, caprine, canine, and various non-human primates.

Some aspects of swine, in particular the organs of the torso were found to be similar to humans (Kent et al 2006, 2008). Studies by Kent et al. $(2006,2008)$ used juvenile porcine subjects to characterize belt loading to the abdominal region. Juvenile porcine subjects were also used as child surrogates to test airbag loading (Aldman et al. 
1974, Mertz et al. 1982, Prasad and Daniel 1984). Additionally, swine have been used as adult human surrogates under blunt impacts to the thorax (Viano and Warner 1976).

Non-human primates have been used as human surrogates for injury biomechanics tests. Baboons were used in child restraint seat tests (Backaitis et al. 1975), airbag severity tests (Mertz et al. 1982) and airbag tests for out-of-position children (Patrick and Nyquist 1972). A study by Schreck \& Patrick (1975) used chimpanzees to test a five-point child restraint harness system. Rhesus monkeys, subjected to blunt impacts to the head and thorax, were studied using high speed cinefluorography (Shatsky et al. 1974), and thoracic force-deflection studies analyzing cardio-thoracic injuries (Beckman and Palmer 1970).

While animals used as human surrogates are genetically different than humans, there are some anatomical similarities that make them useful as injury biomechanics models. Other animal surrogate models include the cervical spine of goats, which was found to be a good model of the human cervical spine due to its vertical orientation (Lu et al. 2005, Zdeblick et al 1993). Canine surrogates have been used to study cardiac injuries from blunt thoracic impact (Roberts et al. 1966, Life and Pince 1967).

There is yet to be a study that considers an animal as a biomechanical model of the pediatric torso as a whole for testing in a seated position and a three-point belted loading condition. The clavicle is an anatomical feature that is an important load path for the shoulder belt restraint. Many quadruped animals frequently used in biomechanics research lack clavicles (e.g. porcine, canine, and caprine). Clavicles help hold the shoulder away from the body and are used for adduction and abduction of the 
arms (Codman 1934). Non-human primates do possess clavicles, but considering the non-human primate subjects used for biomechanics tests in the 1970's the conservation status of the chimpanzee is now categorized as endangered by the International Union for Conservation of Nature and Natural Resources (IUCN 2012). The baboon and rhesus monkey are in the category of least concern; however, the rhesus monkey never reaches the size (by mass) of a human 6-year-old (Nowak 1999, Napier and Napier 1985). To minimize the need for anthropometric scaling, the animal should be approximately the size of the target human 6-year-old. Also, to minimize the need for modulus scaling, the animal model should be similar in size to the human six-year-old when at a similar developmental stage. The baboon is limited as an animal model because it is fully matured when it reaches the size (by mass) of a human six-year-old (Nowak 1999, Napier and Napier 1985, Glassman et al. 1984, Coelho et al. 1984). All of the biomechanical animal models previously used are limited in their utility as a surrogate for the pediatric torso in belted frontal impacts.

\subsection{7: $\quad$ Summary of Limitations of Existing Pediatric Data and Models}

A review of the literature shows there is a limited amount of pediatric biomechanics data available. Of the available data, there are limitations to its usefulness in understanding the thoracic force deformation response of the human 6year-old in a potentially injurious frontal loading with a shoulder belt restraint, since this response is unknown. A summary of the pediatric PMHS and volunteer tests and their limitations are shown in Table 1.1. 
Table 1.1: Limitations of Pediatric PMHS and Volunteer Tests

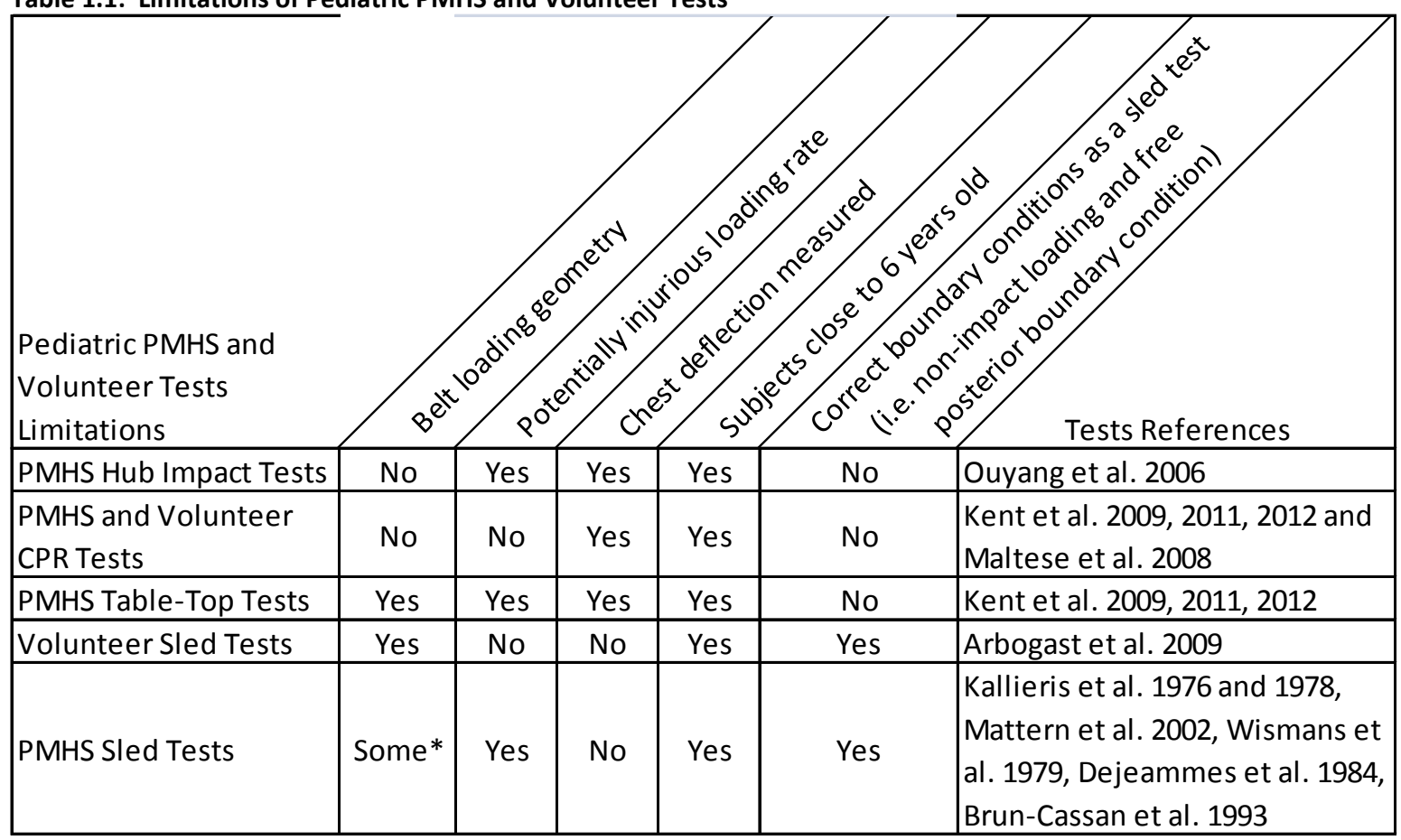

*Only the older subjects had a 3-point belt restraint, younger subjects had a lap shield booster restraint.

Due to the dearth of pediatric biomechanics data, the biofidelity requirements for six-year-old physical ATDs and computational models are based on scaling from adult biofidelity requirements. There is not a direct relationship between mass distribution and material properties between human children and adults. This leads to uncertainty in the scale factors (based on length scale, mass density, and modulus of elasticity ratios) since dimensional scaling involves assumptions of geometric similitude and loading of homologous structures. Overall, the computational models and ATDs are insufficiently developed and validated for reliable use in frontal belt loading.

The majority of animals used in biomechanics research are quadrupeds and they lack clavicles. The non-human primates used in previous biomechanical tests either never reach the size of a human six-year-old, are already mature when at the size of a human six-year-old, or they are endangered. 


\section{3: Justification for Performing a Detailed Biomechanical Assessment of the}

\section{Kangaroo}

Due to the scarcity of pediatric PMHS and testing restrictions of those available, an alternative biomechanical model was considered. A review of previous studies determined that important factors to consider for a potential biomechanical model are availability of the model and geometric, length, mass, and modulus similitude of the model. This study considers the kangaroo as a novel biomechanical model of the pediatric thorax in potentially injurious frontal loading with a seat belt restraint. This section will discuss reasons that merit the assessment of the kangaroo.

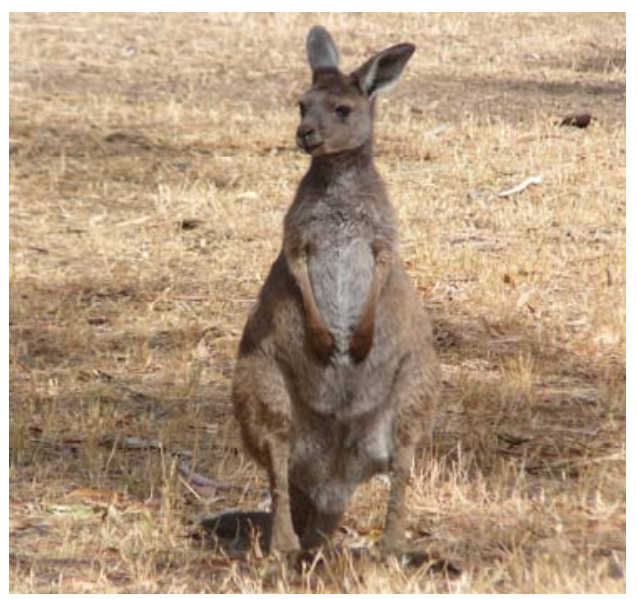

Figure 1.5: Juvenile Eastern Grey Kangaroo standing upright on hind legs

\subsection{1: $\quad$ Availability}

The International Union for Conservation of Nature and Natural Resources reports the conservation status of the kangaroo is in the category of least concern (IUCN 2012)\}. At the start of this project, the abundance of kangaroos in Australia resulted in culling to lower the number of kangaroos because the over population in certain areas caused damage to crops and fences in addition to being road hazards. 


\subsection{2: $\quad$ Geometric Similitude}

Torso bony structures and orientation of the subject are anatomical features considered for geometric similitude. The biomechanical model should be able to be positioned upright and be able to interact with a belt similarly to how a child would be loaded in those conditions.

Humans are bipedal after infancy, while the majority of animals are quadrupeds. Kangaroos are one animal species that occasionally walk or hop bipedally (Alexander 2004). The stature of the animal contributes to the spinal curvature and organ positioning Semi-erect animals compared to quadrupeds would ideally be easier to position in a seated upright position, and be more representative of a human six-yearold, considering the shoulder girdle.

Kangaroos have well developed clavicles due to the need to climb into the pouch after birth (Dawson 1995). Although the shoulder breadth is narrower compared to the caudal region of the trunk (Figure 1.5), the kangaroo is more similar to the human than other animals and it has an important anatomical structure which many other animals lack.

\subsection{3: $\quad$ Size and Modulus Similitude}

The animal surrogate should be similar in size to the human six-year-old to minimize the need for length and mass scaling. Since mechanical properties of tissues change as humans and animals grow and age, it is also important to minimize the need for modulus scaling, such that the animal model should be similar in size to the human six-year-old when at a similar developmental stage. When considering an animal model, 
ideally the age of the animal model should correlate to the ages of the varying developmental stages of humans. To assess the kangaroo, sexual maturity was used to determine its stage of development. Mass was considered when comparing size to developmental growth. Stature is not an optimal parameter for comparison of size since animals do not have a constant erect posture like humans and there is not a consistent or well-defined measurement for body or trunk length among animals to compare with human stature, due to varying body shapes (e.g. tail length included in total body length measurements). On average, a human six-year-old is at $38-50 \%$ of their sexual maturity (Gavan 1953, Rogol et al. 2002, DeLamater and Friedrich 2002, Zacharias and Wurtman 1969, Nowak 1999, Asdell 1964), and has an average mass of $20.5 \mathrm{~kg}$ (Snyder et al. 1977).

The three main species of large kangaroos were considered to find one with a size-development rate closest to the range of humans; the red kangaroo (Macropus rufus), the western grey kangaroo (Macropus fuliginosus) and the eastern grey kangaroo (Macropus giganteus). Western males mature earlier than eastern males of similar size (Poole and Catling 1974). Although fully grown red kangaroos and eastern grey kangaroos are similar in size, the eastern grey kangaroos have a slower growth rate (Frith and Calaby 1969). Of the three kangaroo species, the eastern grey kangaroo species has a size-development rate closest to the range of humans. When the eastern grey kangaroo is at a mass of $20.5 \mathrm{~kg}$, it has reached $28 \%$ of its maturity (Hopwood 1976 , Russell 1974, Asdell 1964, Poole 1982). This data is an approximate range since the sexual development rate of kangaroos is strongly dependent on environmental factors 
and thus highly variable (Kirkpatrick 1967). Considering availability, similar anatomical features, and developmental and size growth, the eastern grey kangaroo seems to be a reasonable animal surrogate for the human six-year-old.

\section{4: Specific Aims}

The goal of this thesis is to evaluate the eastern grey kangaroo (Macropus giganteus) as a model for the pediatric thorax and to identify its strengths and weaknesses as a biomechanical model of the pediatric thorax under frontal loading. The hypothesis of this thesis is that juvenile eastern grey kangaroos respond in a human-like manner to CPR, blunt hub and diagonal belt loading. Human-like is subject to some interpretation and will be described further for each testing condition. To evaluate this hypothesis, four specific aims were conducted:

1. Obtain juvenile eastern grey kangaroo (kangaroo) carcasses and compare the anatomy of the kangaroo to humans.

2. Perform non-injurious CPR loading tests to kangaroo thoraces and compare with existing pediatric CPR data.

3. Compare kangaroo thoracic biomechanics against existing pediatric data in a hub impact loading environment.

4. Evaluate the belt interaction and thoracic biomechanics of juvenile kangaroo carcasses in frontal sled tests. 


\section{5: Summary}

This thesis presents the assessment of juvenile eastern grey kangaroos (Macropus giganteus) as a biomechanical model of the pediatric thorax in frontal loading. This is a novel study since there is no evidence that the kangaroo has been previously used as a biomechanical model of the pediatric thorax. Pediatric PMHS are not readily available for testing, leading to a dearth of thoracic biomechanical data on pediatric subjects. The animal is being assessed as a tool for studying pediatric seatbelt interactions. The kangaroo is similar in size to a six-year-old child when it is at approximately the same developmental stage and it has some anatomical similarities to humans that many biomechanical animal surrogates do not possess (viz. clavicles).

This thesis provides a detailed anatomical comparison between kangaroos and humans. Three different biomechanical loading conditions were imposed on kangaroo thoraces to evaluate their utility as a model of the pediatric thorax under high-speed belt loading. Two of these loading conditions were chosen based on the availability of published comparison data collected on either living children or pediatric PMHS, and included non-injurious cyclic CPR type loading and thoracic hub impacts. Finally, a series of frontal low- and high-speed impact tests with diagonal-belt loading were performed on kangaroo subjects. 


\section{Chapter 2: Kangaroo Subject Acquisition and Anatomy}

\section{1: Introduction}

This chapter describes the acquisition of juvenile kangaroo carcasses, and provides a detailed anatomical comparison between the kangaroo and human.

\section{2: $\quad$ Subject Acquisition}

Twelve (7 female, 5 male) juvenile eastern grey kangaroo carcasses were obtained during a standard culling procedure and imported with approval of the Australian Department of Environment, the U.S. Food and Drug Administration, the University of Virginia Animal Care and Use Committee, and the University of Adelaide Animal Ethics Committee. The subjects were obtained from the southeastern region of South Australia, near Mt. Gambier. To aid in shipping, the lower extremities were amputated mid-tibia, and the majority of the caudal vertebrae (i.e. the tail) were removed to allow for a sitting posture. To minimize damage to the body structure, kangaroo subjects were obtained with a single high-precision shot to the head.

The kangaroos were frozen shortly after death at $-20^{\circ} \mathrm{C}$, and computed tomography (CT) scans were performed on each subject to assure the thorax was structurally intact with no pre-existing fractures. Subjects were scanned at $1 \mathrm{~mm}$ slice thickness at an interval of $0.5 \mathrm{~mm}$ on a Toshiba Aquilion ${ }^{\mathrm{TM}} 16 \mathrm{CT}$ scanner. One subject, $\mathrm{K} 08 \mathrm{M}$, had an existing spiral fracture in the left humerous, but this did not affect its use in the thoracic characterization tests. Blood tests to check for $\mathrm{Q}$ fever were also performed, and all subjects tested negative. 


\section{3: Subject Information}

Table 2.1 provides full subject zoometric measurements and subject information. Thoracic measurements included breadth, depth and circumference at the level of the axilla, the xiphoid process (XP), and the iliac crest. Initial measurements were taken of the subjects when they were frozen. There is some discrepancy in frozen zoometric measurements and thawed subjects due to the contorted position of frozen carcasses. Zoometric measurements were recorded again on thawed subjects prior to their use in experimental tests.

Only seven of the twelve obtained kangaroos were tested, three in the hub impact, and four in sled tests. The non-injurious CPR cyclic loading was performed on the seven subjects prior to the hub impact or sled tests. Measurements on all twelve carcasses were used for the zoometry and anthropometry comparison.

The target size for the kangaroo carcasses was a human six-year-old. The intact mass of the juvenile kangaroo carcasses obtained ranged between approximately the $5^{\text {th }}$ percentile six-year-old and a $95^{\text {th }}$ percentile eight-year-old human (Figure 2.1). The seated height measurement was determined as the seated surface to acromion height since some of the kangaroo subjects did not have a head. Pediatric anthropometric data from Anthrokids (Snyder et al. 1977) did not have this specific measurement, so it was estimated as the suprasternal height minus the gluteal furrow height (Figure 2.3). The kangaroo seated height was closer to a human teenager or older, than to a six-year-old (Figure 2.2). 


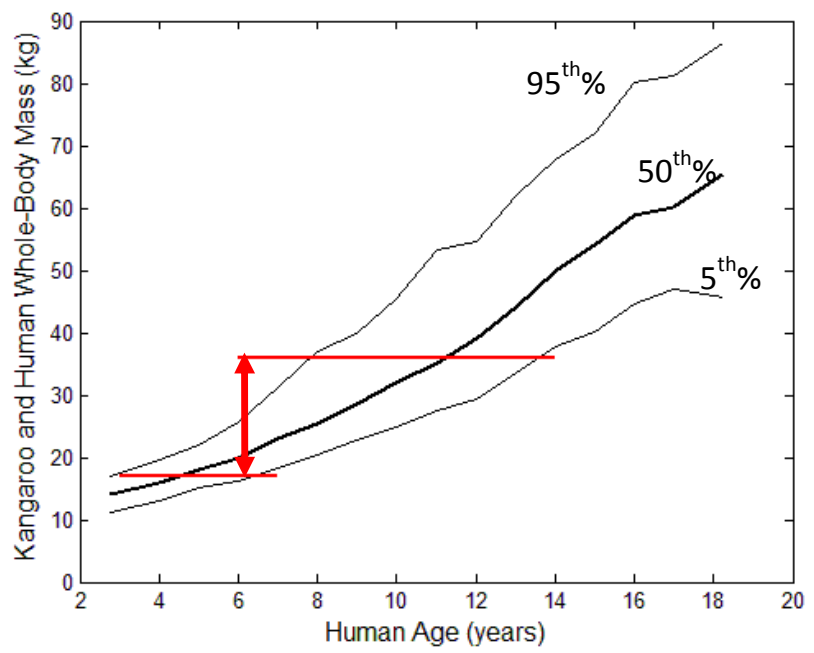

Figure 2.1: Correlation between kangaroo whole-body mass (with tail) and human age equivalent based on Snyder et al. (1977). Red arrow indicates mass range of kangaroo subjects.

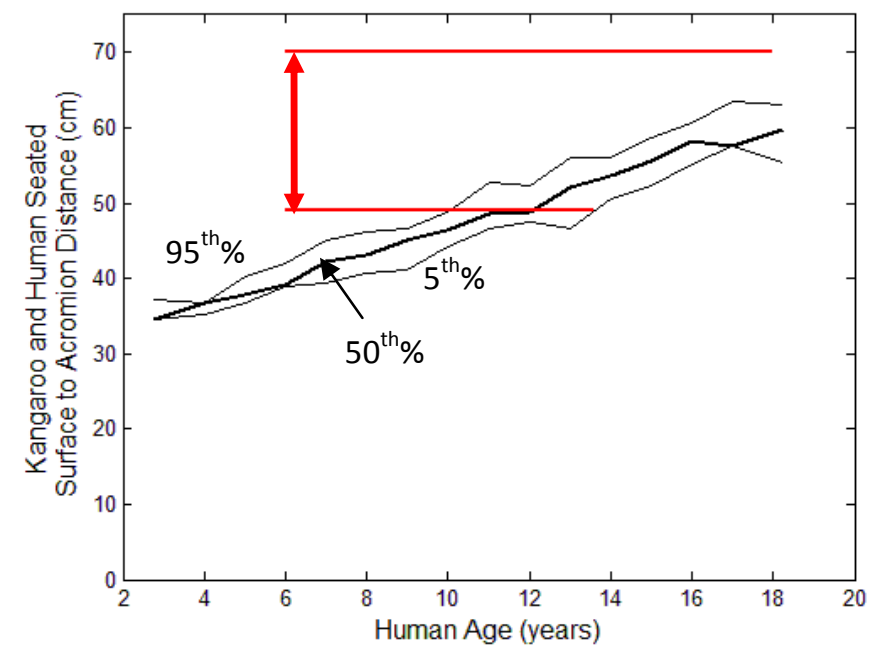

Figure 2.2: Correlation between seated height to the acromion of kangaroo and estimated seated height to acromion based on human age (Snyder et al. 1977). Red arrow indicates seated surface to acromion distance range of kangaroo subjects.
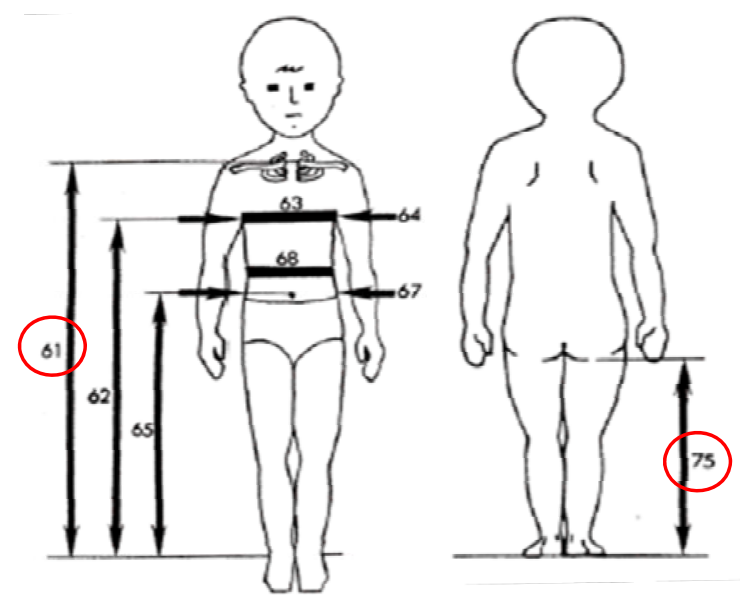

Figure 2.3: Illustration of suprasternal height (61) and gluteal furrow height (75) (Source: Anthrokids) 
Table 2.1: Summary of all kangaroo specimen information and anatomical measurements

\begin{tabular}{|c|c|c|c|c|c|c|c|c|c|c|c|c|}
\hline Subject ID & K01F & K02F & K03F & K04M & K05M & K06F & K07F & K08M & K09F & K10M & K11M & K12F \\
\hline \multicolumn{13}{|c|}{ General Information } \\
\hline Tests performed on subject & CPR, Sled & CPR, Sled & none & none & CPR, Sled & CPR, Sled & none & CPR, Hub & CPR, Hub & CPR, Hub & none & none \\
\hline Sled Test Numbers/Hub impact level & $\begin{array}{c}1433,1434 \\
1435\end{array}$ & $\begin{array}{c}1436,1437 \\
1446\end{array}$ & $\mathrm{n} / \mathrm{a}$ & $\mathrm{n} / \mathrm{a}$ & 1440,1441 & 1438,1439 & $\mathrm{n} / \mathrm{a}$ & $\mathrm{T} 4, \mathrm{XP}$ & $\mathrm{T} 4, \mathrm{XP}$ & $\mathrm{T} 4, \mathrm{XP}$ & $\mathrm{n} / \mathrm{a}$ & $\mathrm{n} / \mathrm{a}$ \\
\hline Sex & $\mathrm{F}$ & $\mathrm{F}$ & $\mathrm{F}$ & $\mathrm{M}$ & $\mathrm{M}$ & $\mathrm{F}$ & $\mathrm{F}$ & $\mathrm{M}$ & $\mathrm{F}$ & $\mathrm{M}$ & $\mathrm{M}$ & $\mathrm{F}$ \\
\hline Significant Anomalies & None & None & None & None & None & None & None & $\begin{array}{c}\text { Frac. left } \\
\text { humerous }\end{array}$ & None & None & None & None \\
\hline Cause of Death & Head Shot & Head Shot & Head Shot & Head Shot & Head Shot & Head Shot & Head Shot & Head Shot & Head Shot & Head Shot & Head Shot & Head Shot \\
\hline Preservation Method & Freezing & Freezing & Freezing & Freezing & Freezing & Freezing & Freezing & Freezing & Freezing & Freezing & Freezing & Freezing \\
\hline Q Fever Assay & Negative & Negative & Negative & Negative & Negative & Negative & Negative & Negative & Negative & Negative & Negative & Negative \\
\hline Relevant Comments & $\begin{array}{c}\text { Sled Trial } \\
\text { Subject }\end{array}$ & None & None & None & None & None & No Head & No Head & No Head & No Head & No Head & No Head \\
\hline \multicolumn{13}{|c|}{ Anthropometry $^{\mathrm{A}}$} \\
\hline Body Mass Intact (kg) & 26 & 26 & 25 & 29 & 31 & 20 & 27 & 33 & 17 & 23 & 36 & 24 \\
\hline Body Mass with amputations $(\mathrm{kg})^{\mathrm{B}}$ & 23 & 22 & 18 & 24 & 25 & 14 & 22 & 26 & 14 & 20 & 28 & 18 \\
\hline Seated Height $(\mathrm{cm})$ & 74 & 76 & 76 & 76 & 82 & 72 & $\mathrm{n} / \mathrm{a}$ & $\mathrm{n} / \mathrm{a}$ & $\mathrm{n} / \mathrm{a}$ & $\mathrm{n} / \mathrm{a}$ & $\mathrm{n} / \mathrm{a}$ & $\mathrm{n} / \mathrm{a}$ \\
\hline Seated surface to acromion $(\mathrm{cm})^{\mathrm{C}}$ & 62 & 59 & 60 & 65 & 69 & 49 & 66 & 67 & 55 & 52 & 70 & 61 \\
\hline Shoulder Breadth $(\mathrm{cm})$ & 9 & 10 & 12 & 12 & 11 & 10 & 11 & 13 & 9 & 11 & 11 & 9 \\
\hline Thorax Breadth (axilla) (cm) & 6 & 14 & $\mathrm{n} / \mathrm{a}$ & 11 & 15 & 11 & 8 & 13 & 10 & 10 & 13 & 9 \\
\hline Thorax Breadth (XP) $(\mathrm{cm})$ & 20 & 20 & 19 & 18 & 22 & 12 & 18 & 24 & 2 & 16 & 22 & 14 \\
\hline Thorax Breadth (iliac crest) $(\mathrm{cm})$ & 20 & 23 & 16 & 25 & 27 & 17 & 22 & 25 & 22 & 17 & 22 & 19 \\
\hline Thorax Depth (axilla) (cm) & 14 & 11 & $\mathrm{n} / \mathrm{a}$ & 11 & 20 & 10 & 10 & 13 & 10 & 11 & 14 & 10 \\
\hline Thorax Depth (XP) (cm) & 25 & 23 & 23 & 17 & 21 & 17 & 20 & 22 & 16 & 16 & 23 & 15 \\
\hline Thorax Depth (iliac crest) $(\mathrm{cm})$ & 28 & 21 & 27 & 25 & 28 & 25 & 19 & 26 & 18 & 20 & 19 & 22 \\
\hline Thorax Circum. (axilla) (cm) & 51 & 45 & $\mathrm{n} / \mathrm{a}$ & 42 & $\mathrm{n} / \mathrm{a}$ & 40 & 37 & 45 & 35 & 38 & 52 & 36 \\
\hline Thorax Circum. (XP) (cm) & 63 & 66 & 60 & 55 & 68 & 49 & 51 & 69 & 53 & 60 & 66 & 50 \\
\hline Thorax Circum. (iliac crest) $(\mathrm{cm})$ & 81 & 79 & 76 & 86 & 75 & 70 & 72 & 81 & 73 & 76 & 76 & 77 \\
\hline
\end{tabular}

Notes:

A - Measurements taken with subjects frozen

$B$ - Mass of subject with tail and leg amputated

$\mathrm{C}$ - Some kangaroo subjects did not have a head to measure a seated height so this measurement was taken for all subjects 


\section{4: Anatomy Comparison}

The morphology of the torso's bony structures is important for the shoulder belt interaction. This section compares the clavicles, scapulae, sternum, rib cage, and vertebrae of kangaroos and humans. Thoracic organ masses and mass distribution were also compared. The CT scans used for the general comparison are from a six-year-old female human, and the male kangaroo subject K08M.

\subsection{1: $\quad$ Bony Structures}

Kangaroos have highly developed shoulders at an early age due to the need to climb into the pouch after birth (Dawson 1995). The kangaroo clavicles are short compared to humans resulting in a narrow shoulder breadth. The kangaroo clavicles have only one convex curvature whereas human clavicles have a convex and concave curvature in the axial plane (Figure 2.4). The acromion process of the kangaroo scapula is not as developed as a human's, but it does protrude past the glenoid which helps in stabilization of the shoulder joint (Figure 2.6 and Figure 2.7).

Both rib cages of the human and kangaroo are conical-shaped, i.e. narrower cranially and wider caudally, but the conical shape is more prominent in the kangaroo. The kangaroo rib cage is more circular than reniform along the axial plane, compared to the human, which positions the scapulae more in the sagittal plane instead of the frontal plane (Figure 2.7).

At the age of six years, the human sternum has not fused and has a total of six bones similar to the kangaroo which has six bones in the sternum including the manubrium and a large cartilaginous xiphoid process (Figure 2.4). The length of the 
kangaroo sternum is longer than the human because each sternabrae bone is long in the cranial-caudal direction compared to the cuboid sternabrae of humans. The sternumrib articulation of the human and kangaroo are the same (i.e. like in the human, the first seven pairs of ribs articulate with the cartilage, with three false ribs attached to the cartilaginous portion of the next above rib, and two floating ribs) with the exception of an additional pair of floating ribs in kangaroos (Sorensen 1906) (Figure 2.6). The kangaroo ribs are very slender and narrow connecting to the sternum with thin and flexible costal cartilage (details in Appendix A).

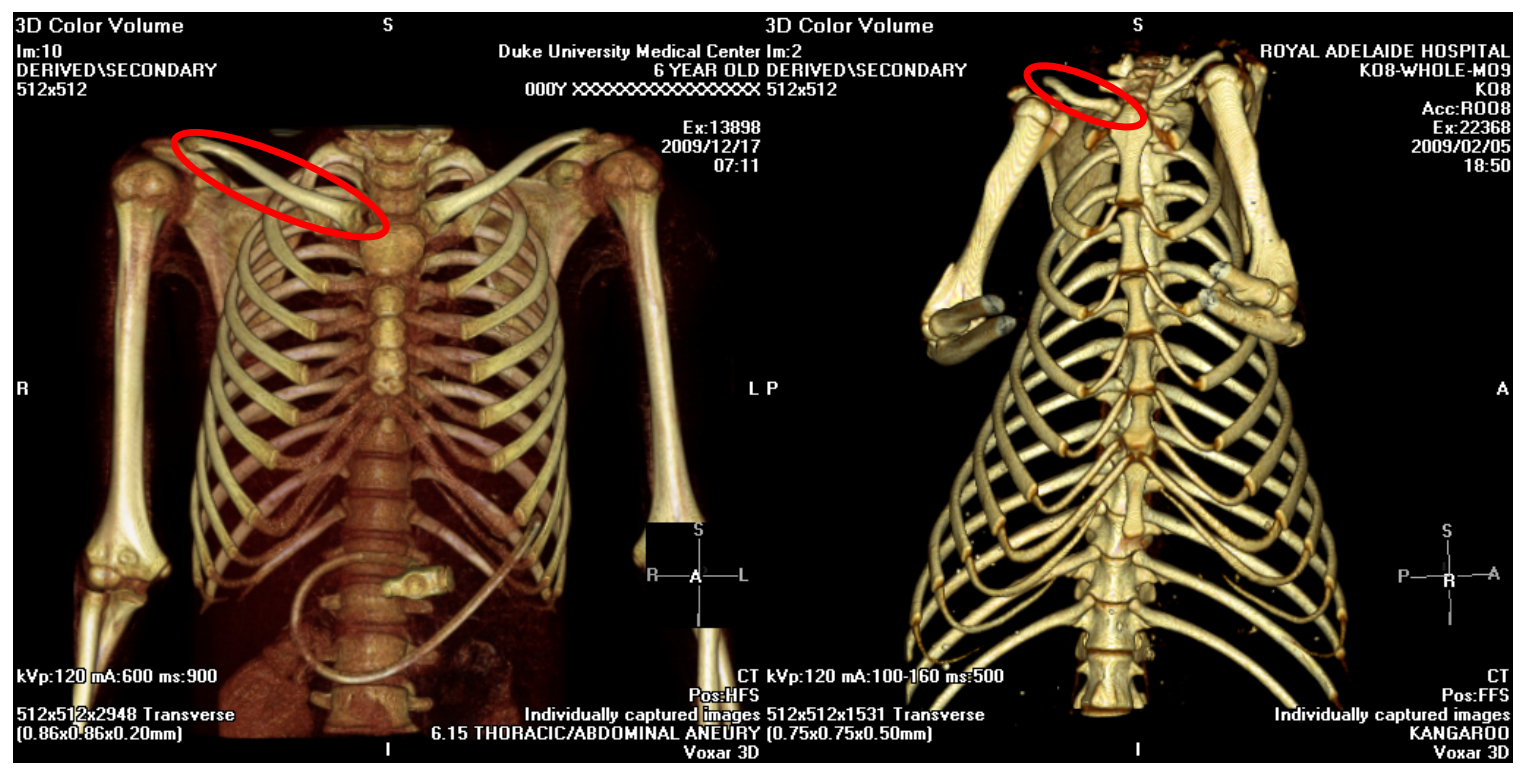

Figure 2.4: Anterior CT images dipicting morphology of a six-year-old human thorax (left) and a juvenile eastern grey kangaroo thorax (right) with the right clavicles highligthed (images not to scale). 


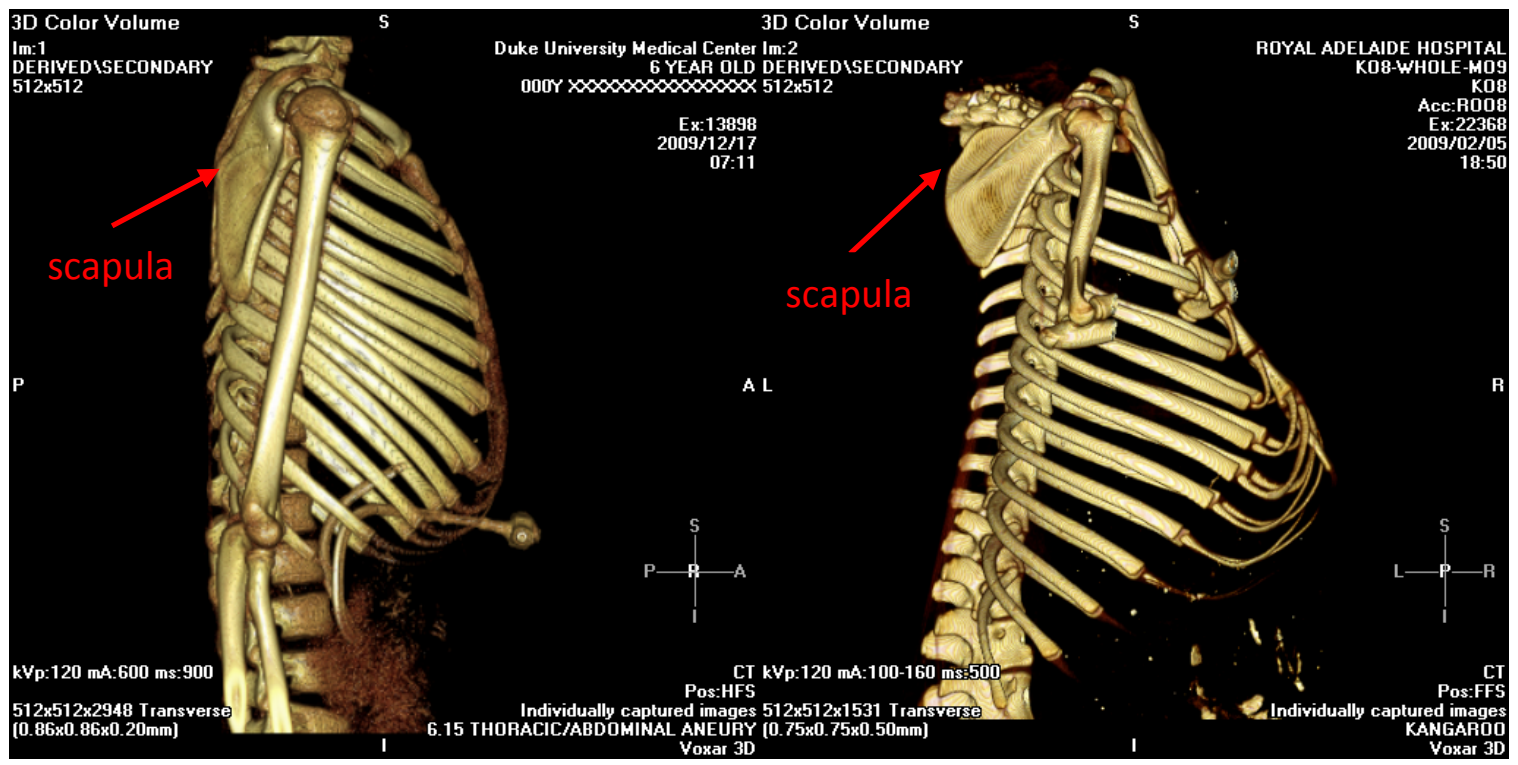

Figure 2.5: Right saggital CT images depicting morphology of a six-year-old human thorax (left) and a juvenile eastern grey kangaroo thorax (right) (images not to scale).

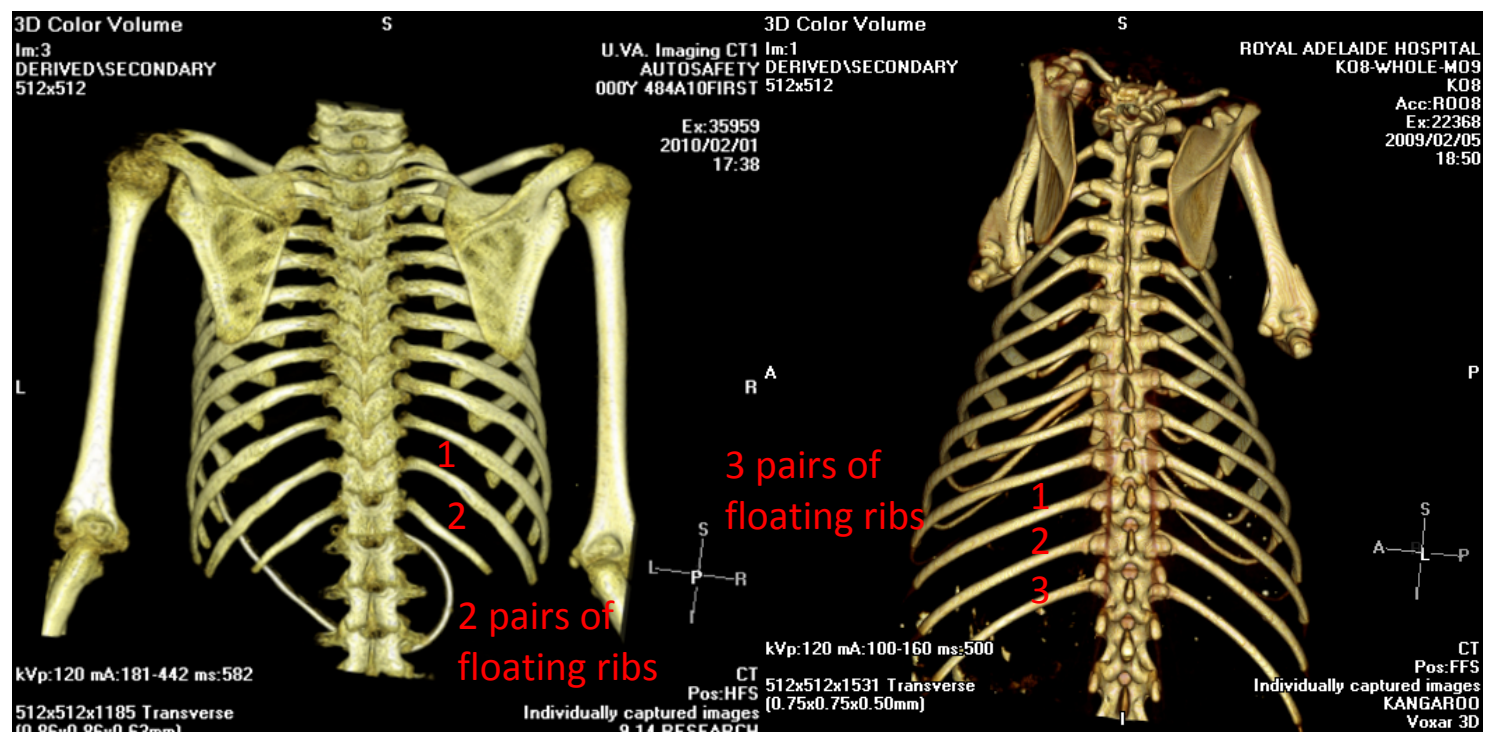

Figure 2.6: Posterior CT images depicting morphology of a six-year-old human thorax (left) and a juvenile eastern grey kangaroo thorax (right) showing the number of floating ribs (images not to scale). 


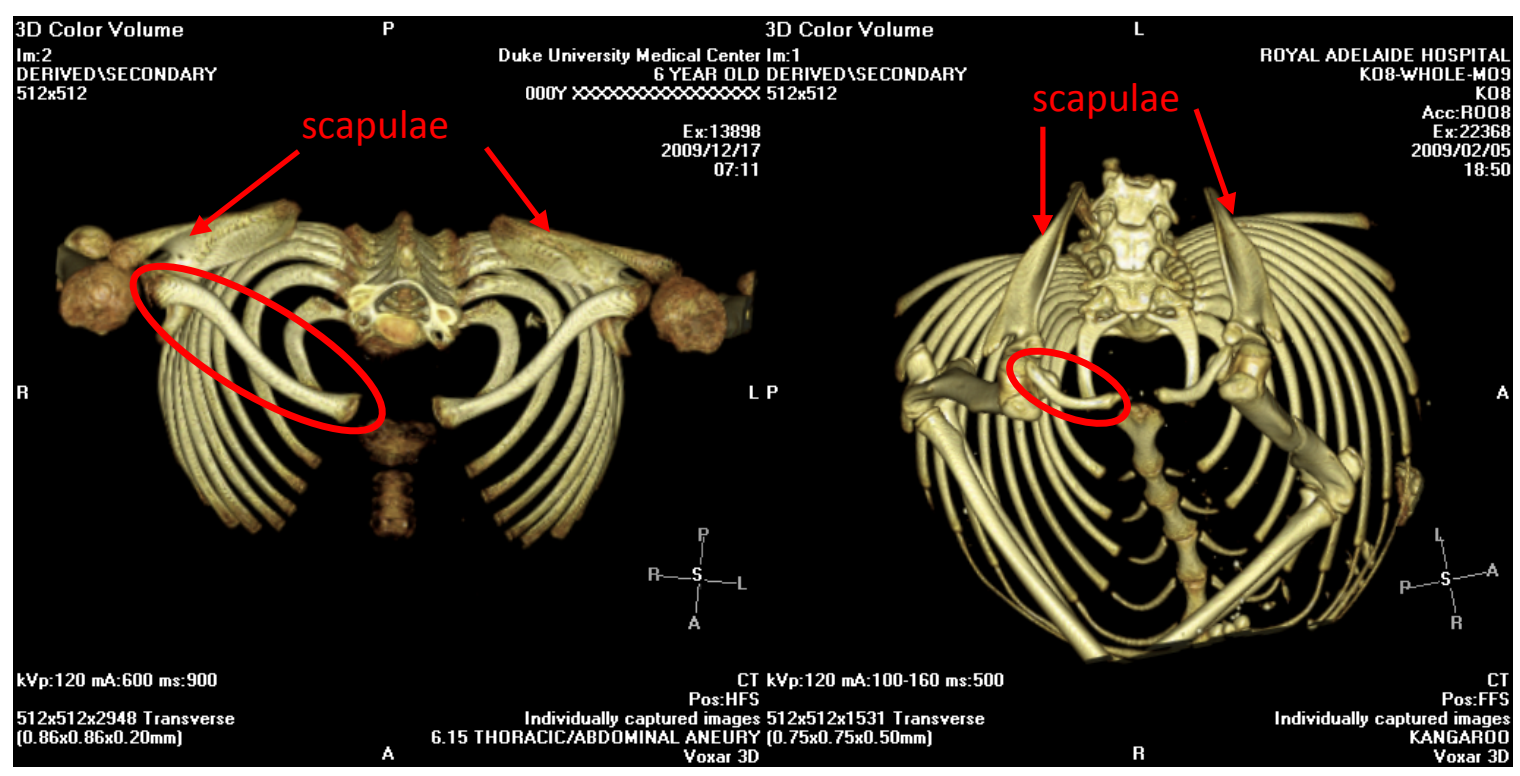

Figure 2.7: Superior CT images depicting morphology of a six-year-old human thorax (left) and a juvenile eastern grey kangaroo thorax (right) with the scapulae and right clavicles highlighted (images not to scale).

Table 2.2 provides the number of cervical, thoracic and lumbar vertebrae for humans and kangaroos. Additionally, the number of ribs corresponds to the number of thoracic vertebrae for each species. There is an additional thoracic and lumbar vertebra in the kangaroo. The quantity of vertebrae creates a longer kangaroo torso in addition to the shape of each vertebra. The kangaroo vertebral bodies are cylindrical and are long and narrow in the cranial-caudal direction. The transverse diameters of human vertebrae are almost twice their height (i.e. they are short and wide Figure 2.8). The length of the kangaroo vertebral bodies produces larger intercostals spacing in kangaroos compared to humans. Humans have an upright posture requiring the Sshaped spinal curvature with a lordotic curve in the lumbar region and a kyphotic curve in the thoracic region. The kangaroo is a semi-erect animal with a C-shaped spinal curvature where the thoracic and lumbar spines are kyphotic. The kangaroo spine continues past the pelvis with caudal vertebrae which aid in balance. 
Table 2.2: Number of vertebrae for humans and kangaroos

\begin{tabular}{|l|c|c|}
\hline & Human & Kangaroo \\
\hline \# C-spine & 7 & 7 \\
\hline \# T-spine/Ribs & 12 & 13 \\
\hline \# L-spine & 5 & 6 \\
\hline
\end{tabular}

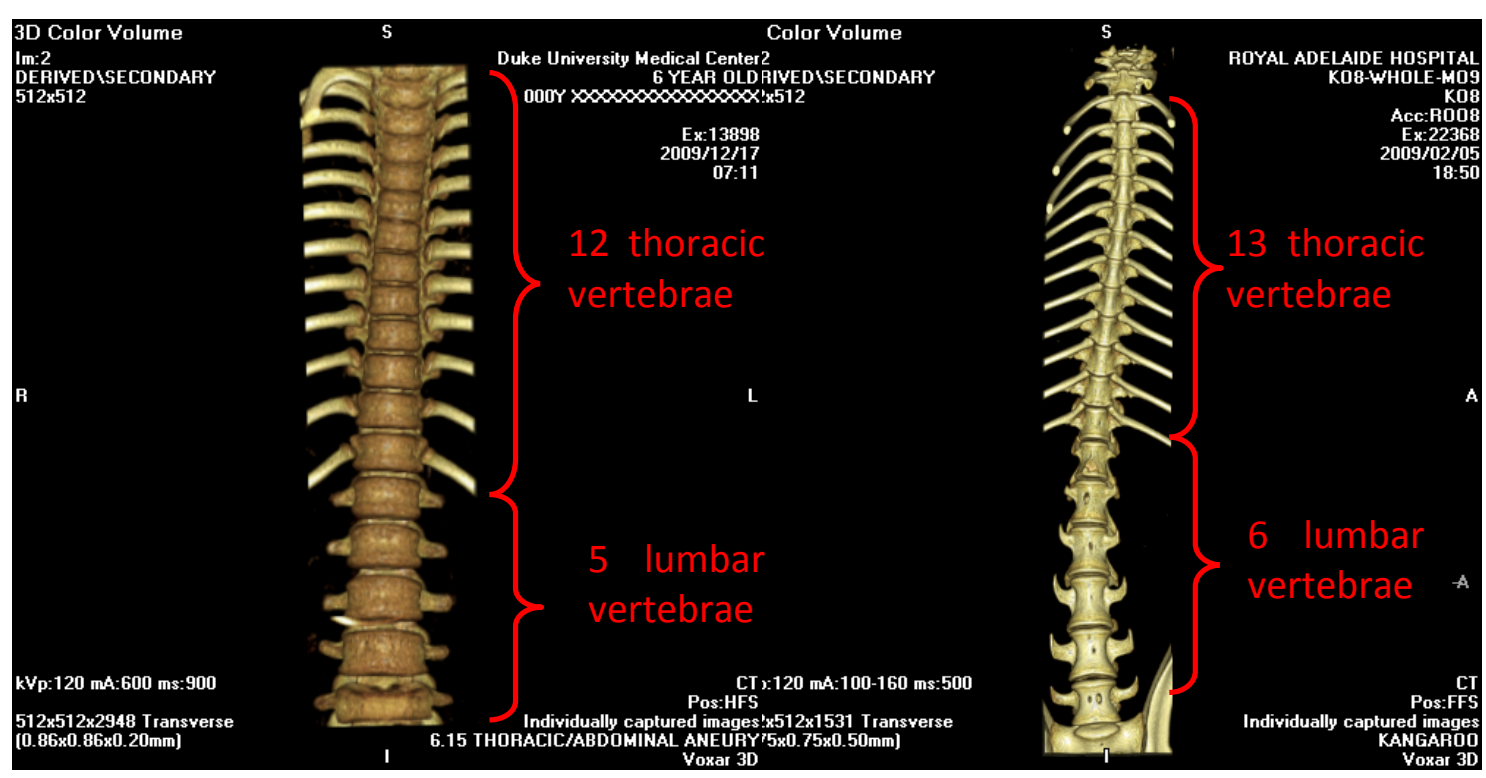

Figure 2.8: Anterior CT images depicting thoracic and lumbar spine morphology of a human six-year-old (left) and a juvenile eastern grey kangaroo (right) (images not to scale).

\subsection{2: $\quad$ Organ and Mass Distribution}

The semi-erect posture of the kangaroo leads to an organ tethering more similar to a human than a quadrupedal animal. Figure 2.9 compares the mass of internal organs in the torso of six-year-old humans and a juvenile kangaroo. The six-year-old data is the average weight of organs with $95^{\text {th }}$ percent confidence intervals for male and female six-year-olds (Stocker and Dehner 2001)) and the juvenile kangaroo data was from one eastern grey subject with a live weight of $21.3 \mathrm{~kg}$ (Tribe and Peele 1963). The kangaroo had smaller lungs, spleen, liver, and kidneys, but the heart was larger. 


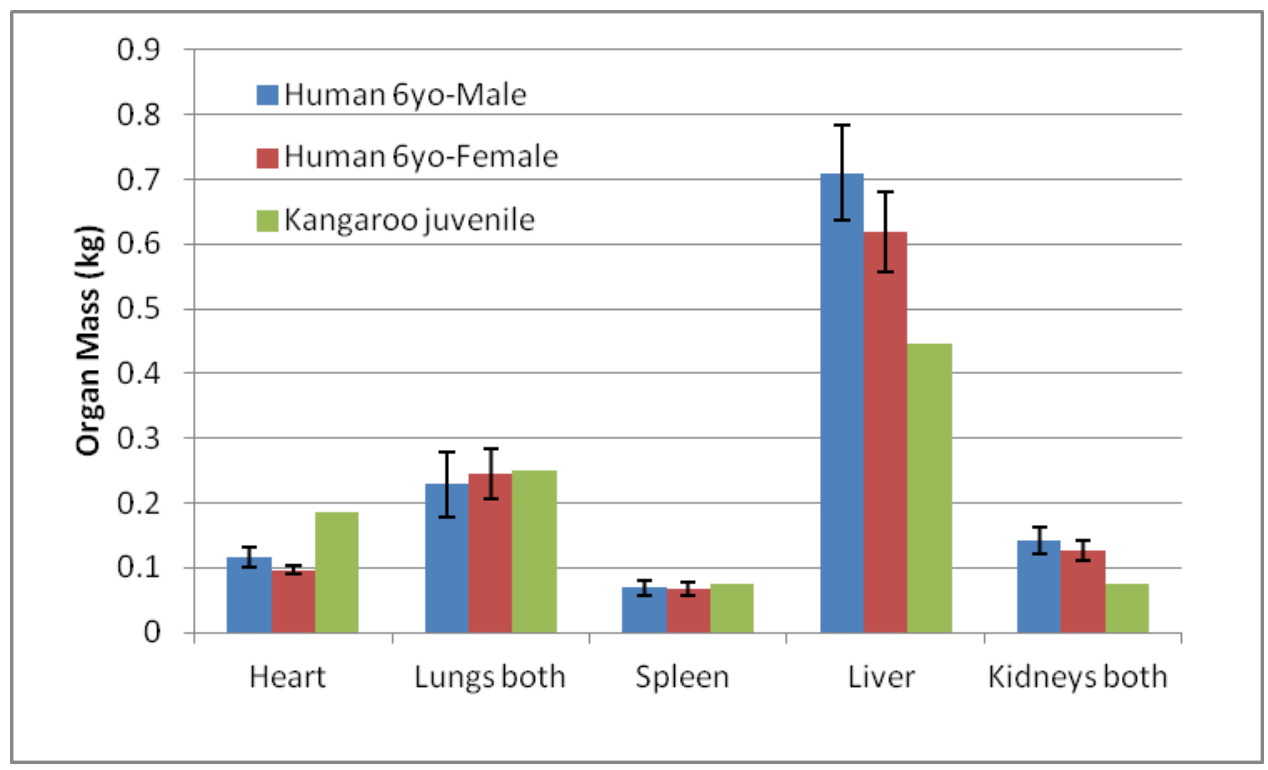

Figure 2.9: Human 6-year-old and juvenile organ mass comparison. Data from Stocker and Dehner 2001, Tribe and Peele 1963.

The mass distribution of the internal organs for a juvenile kangaroo and human six-year-old can be seen in Figure 2.10. The kangaroo has a larger digestive track which shifts the center of gravity of the kangaroo torso more caudal than the human center of gravity.
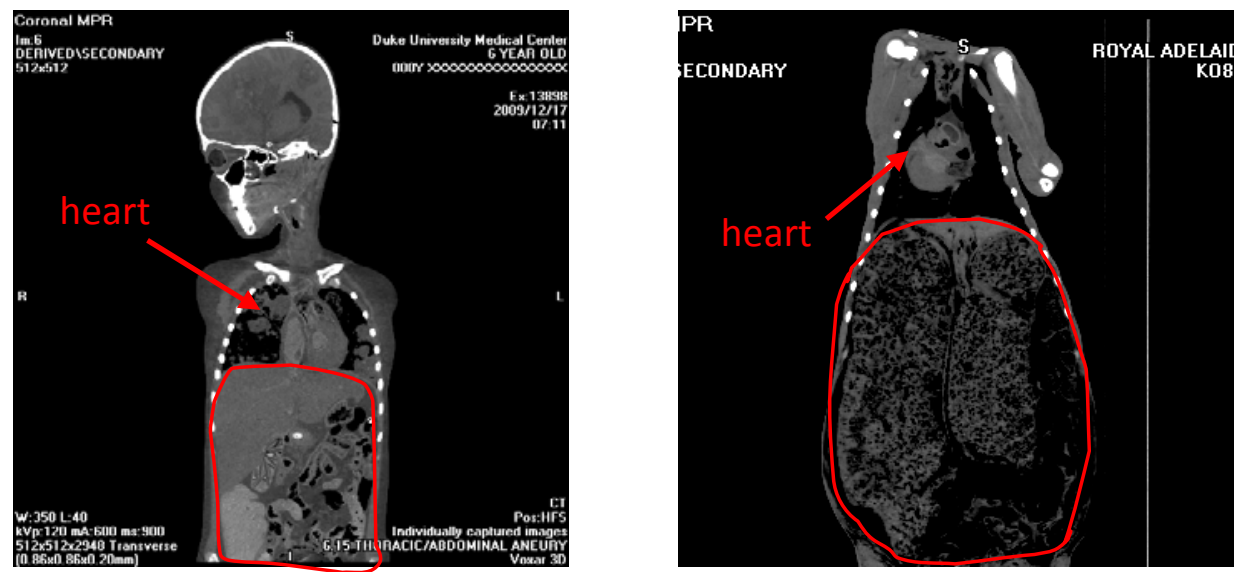

Figure 2.10: CT frontal cross-section of human 6-year-old (left) and juvenile kangaroo (right) showing distribution of internal organs. Red outline of lower thoracic organs and intestinal tract (images not to scale) 


\subsection{3: $\quad$ Zoometry and Anthropometry}

Figure 2.11 and Figure 2.12 show the thoracic breadth and circumference measurements of the twelve juvenile kangaroo subjects obtained compared with anthropometric measurements of average human six-year-olds (Synder et al. 1977). These measurements were taken on the frozen carcasses. Measurements at the level of the axilla and xiphoid process (Table 2.1) for the kangaroos were averaged for the thorax range of data. Kangaroos have short clavicles and subsequently a narrow biacromial breadth compared to broad human shoulders. The pelvic region of the kangaroo is larger compared to humans such that the kangaroo has a more conicalshaped torso compared to humans. The kangaroo mid-thoracic measurements were in the range of human measurements which is ideal for biomechanical thoracic loading tests.

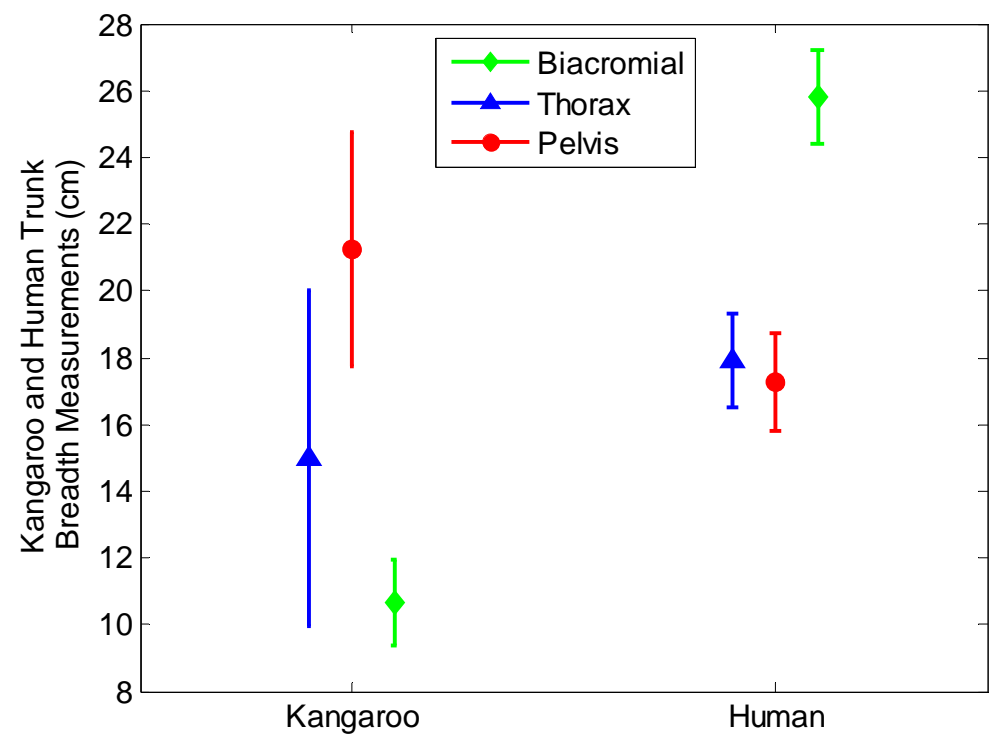

Figure 2.11: Thoracic breadth measurements of juvenile kangaroo subjects and human six-year-olds. 


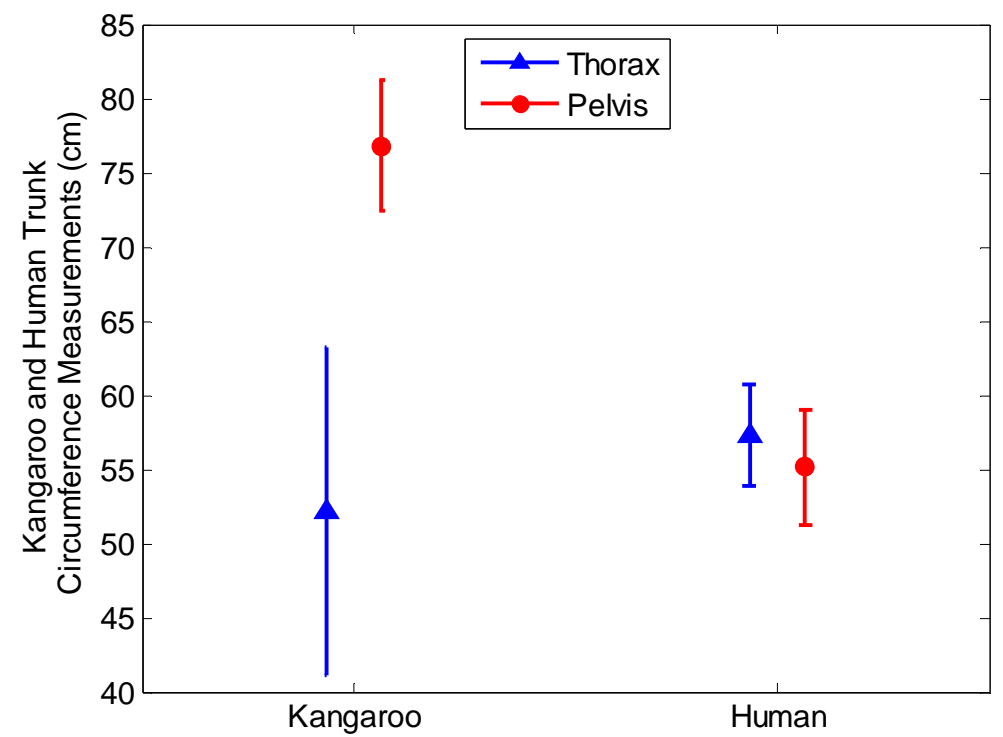

Figure 2.12: Thoracic circumference measurements of juvenile kangaroo subjects and human six-year-olds.

\section{5: Summary}

This chapter describes the procurement of the kangaroos and provides a detailed anatomical comparison between the average six-year-old human and the juvenile eastern grey kangaroos obtained. Key similarities include the presence of clavicles, a six-segmented sternum, and the arrangement of costal cartilage from the rib to the sternum. Key differences include the size, and morphology of the clavicles, the shape of the rib cage in the transverse plane, which is more conical in the kangaroo, the torso length, and distribution of mass. Subsequent chapters in this thesis will describe the biomechanical behavior of the kangaroo's thorax in a number of loading conditions and, where possible, compare it with data from humans. This biomechanical evaluation begins in the next chapter using a CPR loading condition. 


\section{Chapter 3: CPR Thoracic Loading}

\section{1: Introduction and Goals}

The purpose of this study was to assess the eastern grey kangaroo thoracic response under non-injurious CPR loading and to compare it to existing pediatric CPR data. Maltese et al. (2008) performed thoracic CPR cyclic loading on clinical patients, ages 8 to 22 years. Data from the two youngest subjects, 8 and 9 year of age were used for comparison to the kangaroo response. Kent et al. $(2009,2012)$ performed thoracic CPR cyclic loading on three pediatric PMHS, ages 6, 7, and 15 years old. Data from the 6- and 7- year-old PMHS were compared to the kangaroo thoracic response. Thoracic stiffness was analyzed to aid in comparison between the kangaroo, pediatric PMHS and pediatric patients.

\section{2: Methods-Experimental Procedures and Data Analysis}

\subsection{1: $\quad$ Overview and Test Matrix}

The CPR tests were performed on kangaroo subjects prior to their use in the hub test series ( 3 subjects) and the sled test series (4 subjects) (Table 2.1). Each test series was comprised of twenty cyclic compressions at the targeted input values, which were a percentage of the mid-sternum chest depth for each subject. The nominal input was $15 \%$ for the hub test subjects and a series of cycles at $10 \%-15 \%-10 \%$ for the sled test subjects. The first trial subject for the sled tests was only loaded with a nominal $10 \%$ chest depth. The test matrix is provided in Table 3.1 showing each subject's mid-sternal chest depth, the nominal input displacement and the actual input displacement. The 
nominal and actual input displacements are not equal because the system is pressure driven and does not have explicit displacement control.

Table 3.1: Kangaroo CPR Test Matrix

\begin{tabular}{|c|c|c|c|c|c|c|}
\hline Test Name & Subject & $\begin{array}{l}\text { Nom. Disp } \\
\text { Input (mm) }\end{array}$ & $\begin{array}{l}\text { Nom. Disp Input } \\
\text { (\% chest depth) }\end{array}$ & $\begin{array}{l}\text { Chest depth } \\
(\mathrm{mm})\end{array}$ & $\begin{array}{l}\text { Actual Disp } \\
\text { Input (mm) }\end{array}$ & $\begin{array}{c}\text { Actual \% } \\
\text { Disp Input }\end{array}$ \\
\hline CPR_K01F & $\mathrm{K} 01 \mathrm{~F}$ & 13 & 10 & 130 & 12.38 & 9.52 \\
\hline CPR_K02F_1 & $\mathrm{KO2F}$ & 13 & 10 & 130 & 13.44 & 10.34 \\
\hline CPR_K02F_2 & $\mathrm{K} 02 \mathrm{~F}$ & 19.5 & 15 & 130 & 11.63 & 8.95 \\
\hline CPR_K02F_3 & $\mathrm{K} 02 \mathrm{~F}$ & 13 & 10 & 130 & 9.51 & 7.31 \\
\hline CPR_K06F_1 & $\mathrm{K} 06 \mathrm{~F}$ & 10 & 10 & 100 & 6.16 & 6.16 \\
\hline CPR_K06F_2 & $\mathrm{K} 06 \mathrm{~F}$ & 15 & 15 & 100 & 8.94 & 8.94 \\
\hline CPR_K06F_3 & K06F & 10 & 10 & 100 & 7.56 & 7.56 \\
\hline CPR_K05M_1 & K05M & 14 & 10 & 140 & 9.88 & 7.05 \\
\hline CPR_K05M_2 & K05M & 21 & 15 & 140 & 13.88 & 9.91 \\
\hline CPR_K05M_3 & K05M & 14 & 10 & 140 & 10.18 & 7.27 \\
\hline CPR_K08M & K08M & 15 & 10.7 & 140 & 11.94 & 8.53 \\
\hline CPR_K09F & K09F & 15 & 15 & 100 & 8.95 & 8.95 \\
\hline CPR_K10M & K10M & 15 & 12.5 & 120 & 10.60 & 8.83 \\
\hline
\end{tabular}

† actual displacement (\% displacement) at peak of first CPR cycle

\subsection{2: $\quad$ Mechanical Equipment/ Test Hardware}

A pneumatically powered open-loop controlled CPR chest compression machine (Thumper 1005, Michigan Instruments Inc., Grand Rapids, MI) was used for all tests to administer controlled cyclic thoracic compressions to the subjects at 100 cycles per minute (Figure 3.1). The Thumper consists of a pneumatic piston-cylinder arrangement attached to a stiff load frame. The Thumper machine and a metal plate surface were rigidly attached to fixed surfaces in the hub and sled test series.

A load cell and accelerometer force-deflection sensor (FDS) (Philips Healthcare, Andover, MA) puck (Figure 3.1) was placed between the Thumper piston head and the subject mid sternum. This was the same FDS puck used in the CPR tests performed by 
Maltese et al. (2008) and Kent et al. $(2009,2012)$ used to replicate the contact surface area. The FDS sensor ( $127 \mathrm{~mm}$ long $\times 52 \mathrm{~mm}$ wide $\times 24 \mathrm{~mm}$ thick) was adhered to the chest with double stick tape. An approximately $1 \mathrm{~mm}$ thick foam double-stick tape was used for the hub test series subjects. A thinner double-stick tape was used for the sled test subjects. The FDS puck data was recorded at $250 \mathrm{~Hz}$ using the Heartstart MRX CPRsensor utilities program (Laerdals Medical).
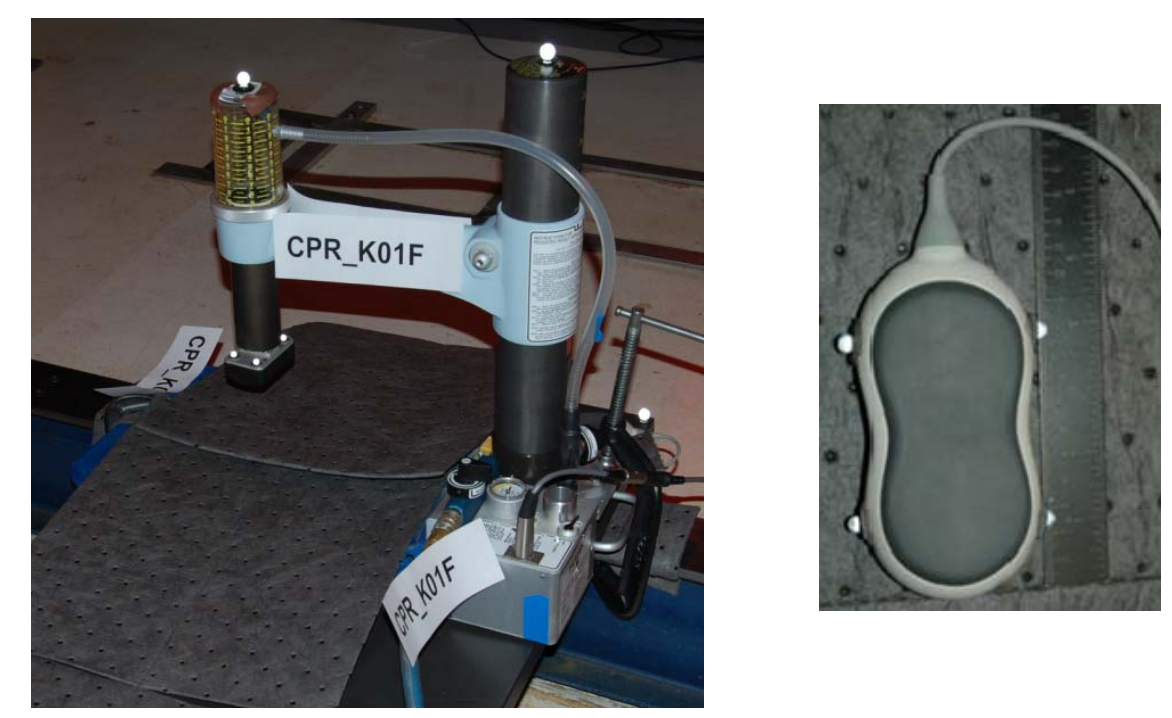

Figure 3.1: CPR Thumper device (left) and force-deflection sensor (FDS puck) (right)

\subsection{1: $\quad$ Subject Preparation and Positioning}

Subjects were thawed at room temperature for 1-2 days prior to testing. The fur on the thorax was sheared to aid in positioning the puck. Each subject was positioned supine on the metal plate and centered beneath the Thumper head such that the FDS puck was centered mid-sternum along the centerline. Blocks were placed around the pelvis region of some subjects to prevent axial rotation of the subject out-of-position. The horizontal piston head frame was adjustable to accommodate subjects with varying 
chest depths. Subjects were palpated after the CPR loading tests to check for rib fractures.

\subsection{2: $\quad$ Test Series Alterations and Improvements}

Slight alterations were made in the test set-up between the CPR compression tests that were run on hub test subjects compared to sled test subjects. A uni-axial load cell and an accelerometer (Endevco model 7264B) were positioned between the FDS puck and the Thumper indenter head for the hub test subjects (Figure 3.2). The force data was obtained from the uni-axial load cell for the hub subjects and from the puck for the sled test subjects.
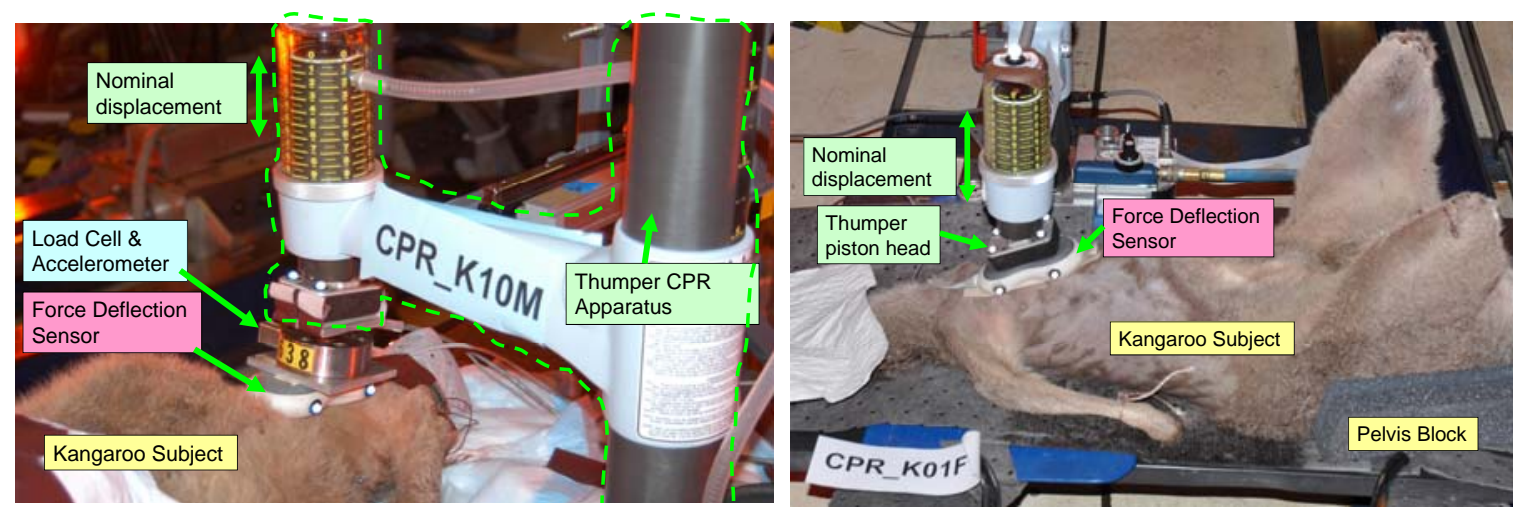

Figure 3.2: CPR FDS puck configuration for hub test subjects (left) and sled test subjects (right)

Subject K06F had a concave sternum, creating a gap between the sternum and the FDS puck. For the tests on subject K06F, the puck was positioned up-side down with the rounded side of the puck facing towards the sternum to accommodate the concavity of the sternum (Figure 3.3). 

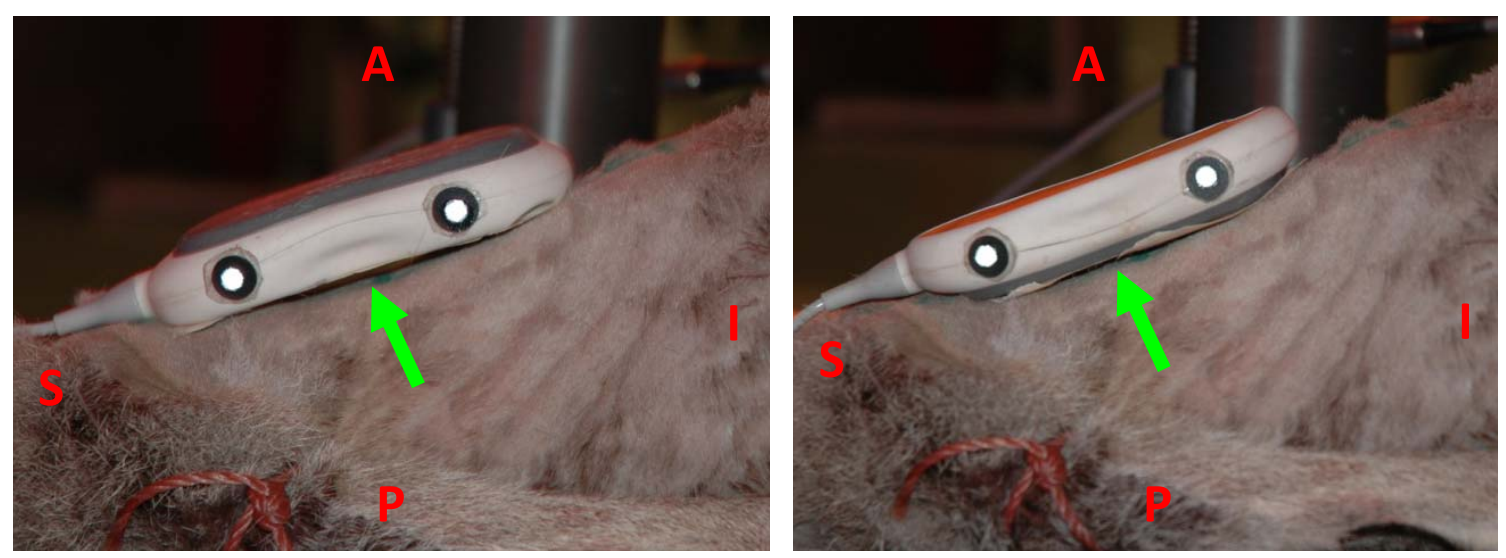

Figure 3.3: Re-orienting FDS puck for subject K06F due to the concavity of the sternum

\section{3: Data Analysis and Comparison}

\subsection{1: $\quad$ Data Collection}

Kinematic chest deflection data was collected using a Vicon $\mathrm{MX}^{\mathrm{TM}} 3 \mathrm{D}$ motion capture system (VICON, Oxford, UK). Retro-reflective markers were placed on the Thumper frame, the piston head, and the FDS puck within a calibrated 3D space of 16 Vicon cameras (Lessley et al. 2010, 2011). Tracking marker arrays in which the markers maintained the same position with respect to one another allowed for the use of a technique that reduced error in the position of the coordinate system created from the individual marker data. The least squares pose estimator algorithm was used to reduce noise in measured marker data using the fixed relative positions of the markers in the array as a physical constraint (Cappozzo et al., 1997).

The data were reported in accordance with the SAE occupant coordinate system, in which the positive z-axis was directed caudally along the spine, the positive $x$-axis was directed perpendicularly to the spine and towards the sternum, and the $y$-axis was perpendicular to the $x-z$ plane directed from the subject's left to right. The $x$-axis 
component of the FDS puck motion was used as the chest deflection measurement. The sampling rate for Vicon was $1000 \mathrm{~Hz}$ and the FDS puck sampling rate was $250 \mathrm{~Hz}$, so the deflection data were decimated to have the same frequency as the force data for each test. Since the force and deflection were measured using two different systems, there was no unified triggering system; therefore, both sets of cyclic data were aligned temporally.

\subsection{2: $\quad$ Data Comparison Analysis}

The stiffness analysis performed by Maltese et al. (2008) on pediatric patients was replicated for the kangaroo and pediatric PMHS data. A simple linear measurement of stiffness can be calculated by the force at maximum deflection divided by the maximum deflection. The stiffness analysis by Maltese et al. (2008) provides a better estimation of thoracic mechanics by fitting a model to the whole force-deflection loop. The clinical pediatric subjects had 656 compressions for the 8-year-old and 1955 compressions for the 9-year-old; whereas, the kangaroo and pediatric PMHS tests had only 20 cycles for each test. Both pediatric PMHS subjects had 3 sets of 20 cycles each. The nominal inputs and actual displacement inputs for the pediatric PMHS are show in Table 3.2. The kangaroo and pediatric PMHS CPR data were both processed with the same method used by Maltese et al. (2008) for comparison against each other and to compare with the pediatric patient data. 
Table 3.2: Pediatric PMHS CPR Test Matrix (Kent et al. 2012)

\begin{tabular}{|l|c|c|c|c|c|c|c|}
\hline Test Name & Subject & $\begin{array}{c}\text { Age } \\
\text { (years) }\end{array}$ & $\begin{array}{c}\text { Nom. Disp } \\
\text { Input (mm) }\end{array}$ & $\begin{array}{c}\text { Nom. Disp } \\
\text { Input (\% } \\
\text { chest depth) }\end{array}$ & $\begin{array}{c}\text { Chest } \\
\text { Depth } \\
\text { (mm) }\end{array}$ & $\begin{array}{c}\text { Actual } \\
\text { Disp.+ } \\
\text { (mm) }\end{array}$ & $\begin{array}{c}\text { Actual \% } \\
\text { Disp.+ }\end{array}$ \\
\hline PEDVE04 & 470F & 7 & 15 & 9.68 & 155 & 11.37 & 7.34 \\
\hline PEDVE05 & $470 F$ & 7 & 30 & 19.35 & 155 & 17.04 & 10.99 \\
\hline PEDVE06 & 470F & 7 & 15 & 9.68 & 155 & 8.37 & 5.40 \\
\hline PEDVE021 & 484F & 6 & 14.2 & 10 & 142 & 13.31 & 9.37 \\
\hline PEDVE022 & 484F & 6 & 21.3 & 15 & 142 & 18.57 & 13.08 \\
\hline PEDVE023 & 484F & 6 & 14.2 & 10 & 142 & 13.08 & 9.21 \\
\hline
\end{tabular}

$\dagger$ actual displacement (\% displacement) at peak of first CPR cycle

To compare the kangaroo CPR data to existing data, a thoracic stiffness analysis was performed on the CPR data which included the applied force through the FDS puck, and the displacement of the puck, $x_{s,}$. Subject chest deflection was defined as $\Delta x=x_{s}$ since the posterior boundary condition is fixed (Figure 3.4).

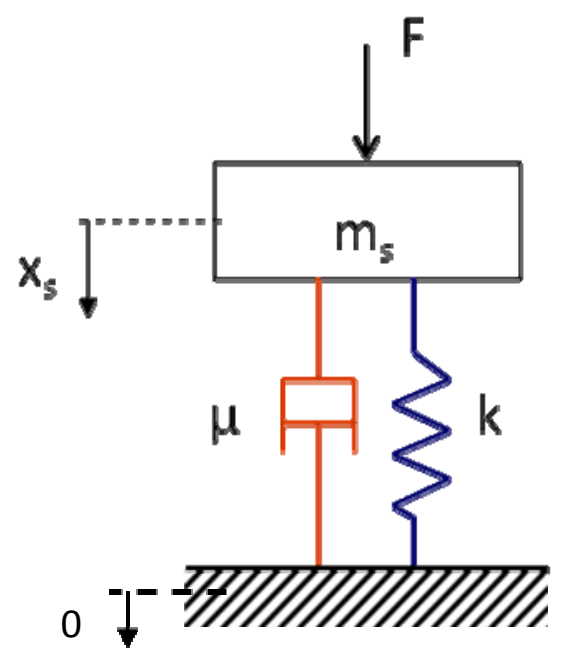

Figure 3.4: Lumped mass model of the chest used for CPR analysis. $F$ is the time-history of the force applied to the chest during CPR, $k$ is the elastic coefficient of the chest, $\mu$ is the viscous coefficient of the chest, $m_{s}$ is the mass of the sternum and $x_{s}$ is the displacement time-history of the sternum.

The torso of the CPR subjects did not fully recover after each loading cycle. This observation will be termed a "molding effect", such that each compression cycle did not begin at the initial $x_{s}$. To accommodate for the "molding effect", the deflection of each curve was zeroed for every cycle prior to fitting a simple lumped mass model (Figure 
3.4). This model, used by Maltese et al. (2008) was used to determine the stiffness ( $k$ ) and damping $(\mu)$ of the chest with the following equation of motion,

$$
m_{s} \ddot{x}_{s}=F-(k \Delta x+\mu \Delta \dot{x})
$$

Equation 3.1

where the sternal mass $\left(m_{s}\right)$ multiplied by the sternal acceleration $\left(\ddot{x}_{s}\right)$ is the inertial force, and $F$ is the force through the FDS puck. Stiffness $(k)$ and damping $(\mu)$ are linearly dependent on chest deflection using elastic $\left(a_{1}, a_{2}\right)$ and viscous coefficients $\left(b_{1}, b_{2}\right)$.

$$
k=a_{1}+\Delta x a_{2}
$$

Equation 3.2

$$
\mu=b_{1}+\Delta x b_{2}
$$

Equation 3.3

The puck had a low acceleration $\left(6 \mathrm{~m} / \mathrm{s}^{2}=0.6 \mathrm{~g}\right)$ and did not impact the sternum, so the inertial force from the accelerated sternal mass of the torso was neglected. The sternal mass is also unknown. Equation 3.1, substituting Equation 3.2 and Equation 3.3, was rewritten as,

$$
a_{1} \Delta x+a_{2} \Delta x^{2}+b_{1} \Delta \dot{x}+b_{2} \Delta x \Delta \dot{x}=F
$$

Equation 3.4

or in matrix form for each time step,

$$
\left[\begin{array}{cccc}
\Delta x\left(t_{0}\right) & \Delta x^{2}\left(t_{0}\right) & \Delta \dot{x}\left(t_{0}\right) & \Delta x\left(t_{0}\right) \Delta \dot{x}\left(t_{0}\right) \\
\Delta x\left(t_{1}\right) & \Delta x^{2}\left(t_{1}\right) & \Delta \dot{x}\left(t_{1}\right) & \Delta x\left(t_{1}\right) \Delta \dot{x}\left(t_{1}\right) \\
\vdots & \vdots & \vdots & \vdots \\
\Delta x\left(t_{n}\right) & \Delta x^{2}\left(t_{n}\right) & \Delta \dot{x}\left(t_{n}\right) & \Delta x\left(t_{n}\right) \Delta \dot{x}\left(t_{n}\right)
\end{array}\right]\left[\begin{array}{c}
a_{1} \\
a_{2} \\
b_{1} \\
b_{2}
\end{array}\right]=\left[\begin{array}{c}
F\left(t_{0}\right) \\
F\left(t_{1}\right) \\
\vdots \\
F\left(t_{n}\right)
\end{array}\right]
$$

Equation 3.5 
The elastic and viscous coefficients were solved using a least squares approach. First the kangaroo and pediatric PMHS CPR tests were compared by determining the elastic force from the model fit at $10 \%$ of each subject's chest depth. At the point of maximum deflection, the sternum velocity is zero, so the viscous forces are neglected from Equation 3.4 to solve for the elastic force. This provided a consistent comparison parameter.

The stiffness was calculated at the apex of each cycle for each test using Equation 3.2 where $\Delta x$ is the peak deflection. The averages of the kangaroo and pediatric PMHS stiffness per cycle number were compared.

The pediatric PMHS and kangaroo force-deflection curves were plotted with force-deflection data from Maltese et al. (2008). Due to the large amount of data from each patient, only the force at maximum chest deflection and the corresponding deflection were plotted with the elastic force curve from the lumped mass model.

\section{4: $\quad$ Results}

\subsection{1: $\quad$ Experimental Results}

It was difficult engaging the kangaroo thorax with the FDS puck because of the conical shape of the torso. Placing the subject supine with the vertebrae directly on the horizontal loading surface resulted in a slanted sternum for the placement of the FDS puck (Table 3.3 and Figure 3.3). Obtaining the target nominal input displacement was difficult for the kangaroo and pediatric PMHS tests due to the dynamics of the Thumper system. Almost all of the actual displacements were less than the nominal displacements (Table 3.1). No fractures were found during palpitations after each test. 
Table 3.3: Kangaroo subject's sternum angle in CPR tests

\begin{tabular}{|l|c|c|c|c|c|c|c|}
\hline Subject ID & K01F & K02F & K05M & K06F & K08M & K09F & K10M \\
\hline Sternum Angle $\left(^{\circ}\right)$ & 25.1 & 19 & 22.9 & 22.5 & 20.7 & 19.8 & 12 \\
\hline
\end{tabular}

The kangaroo force-deflection response appears less stiff (more deflection for a given force) due to molding effect of the thorax (Figure 3.5, top). However, when the deflection of each cycle is zeroed (Figure 3.5, bottom), the force-deflection response of the two species is similar. The molding effect is more prominent in the kangaroo tests compared to the pediatric PMHS tests. The chest depths of the pediatric PMHS subjects were greater than the kangaroo subjects so the pediatric PMHS subjects had greater deflection inputs and resulting greater force responses.
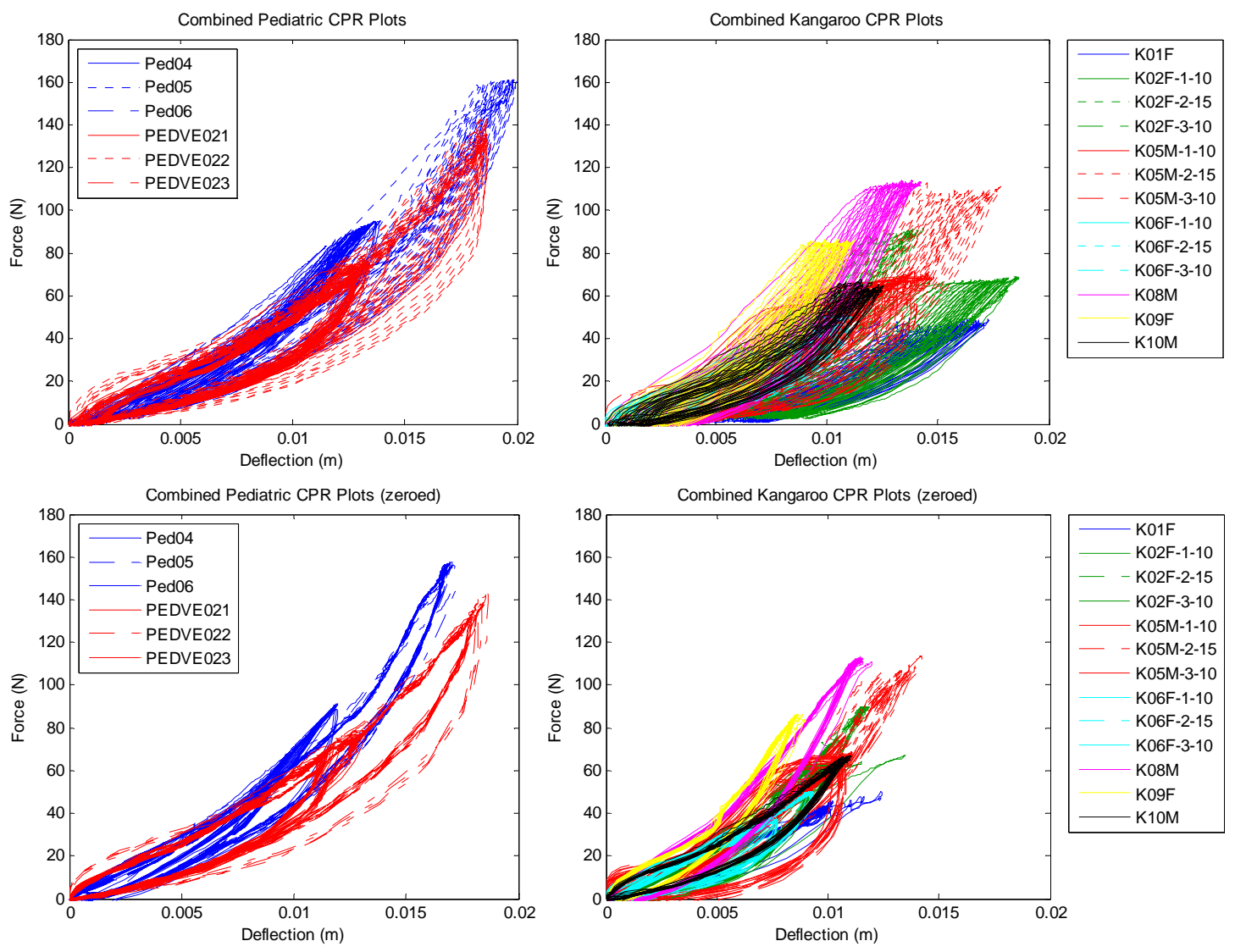

Figure 3.5: Individual force-deflection curves for kangaroo subjects compared to pediatric 6yo and 7yo PMHS (top), and plots with cycles zeroed with respect to deflection (bottom). Legend indicates test ID (see Table 3.1 and Table 3.2) 


\subsection{2: $\quad$ Stiffness Analysis Results}

The elastic force $F_{e}=a_{1} \Delta x+a_{2} \Delta x^{2}$ (from Equation 3.4) at 10\% of each subject's chest depth is shown in Figure 3.6. The force-deflection curves and elastic force from the model are provided for each test in Appendix B, along with calculated coefficient values. The pediatric PMHS have larger chest depths compared to the kangaroos, and show an increasing trend of elastic force with respect to age and chest depth. The kangaroo subjects also show a general increasing trend of elastic force with increasing chest depth, although, there was more variation in the elastic force of the kangaroos.

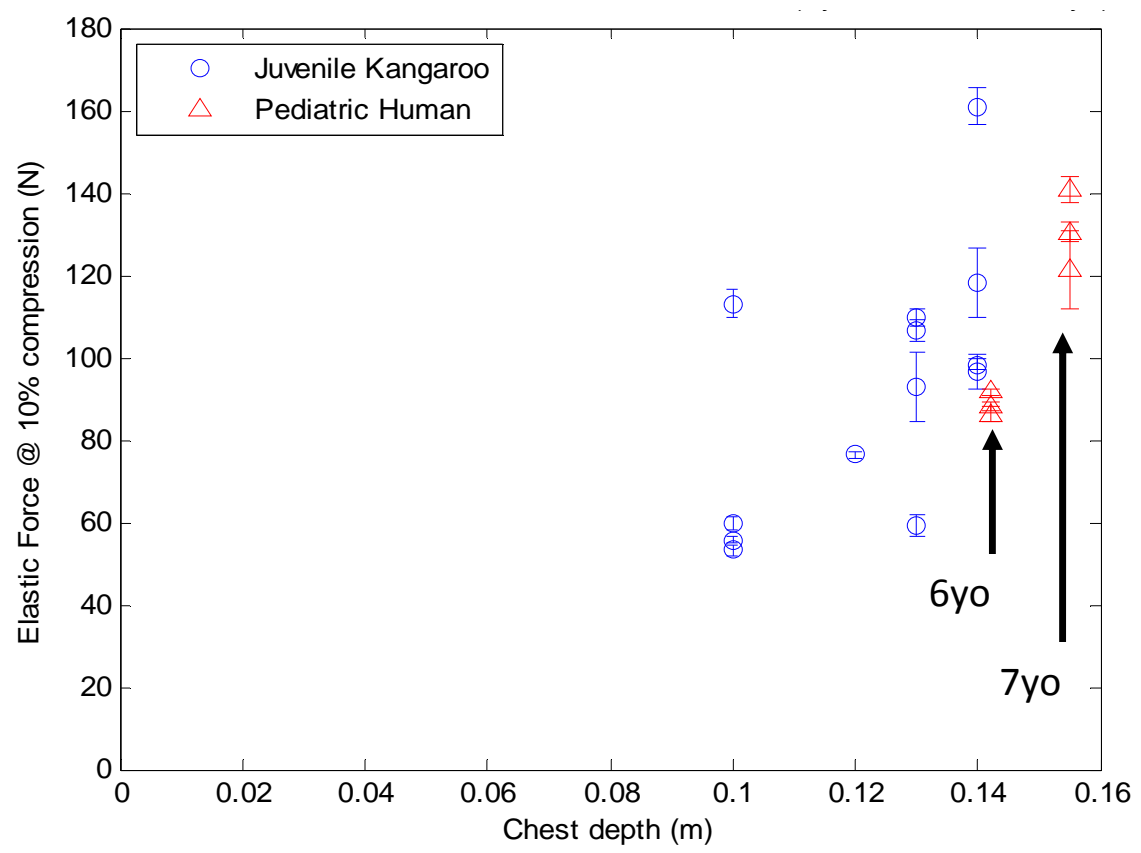

Figure 3.6: Elastic force at $10 \%$ of chest depth for juvenile kangaroo and pediatric PMHS CPR loading tests.

Figure 3.7 shows the average stiffness, $k$, per cycle for each species is comparable. The calculated stiffness for the kangaroos increases as the cycle number increases; whereas the pediatric stiffness changes less over the cycles. The pediatric PMHS had an average stiffness of $7360 \pm 142 \mathrm{~N} / \mathrm{m}$ and average dampening of $93 \pm 17.5$ 
$\mathrm{Ns} / \mathrm{m}$. The kangaroo had an average stiffness of $7481 \pm 442 \mathrm{~N} / \mathrm{m}$ and average dampening of $79 \pm 8 \mathrm{Ns} / \mathrm{m}$.

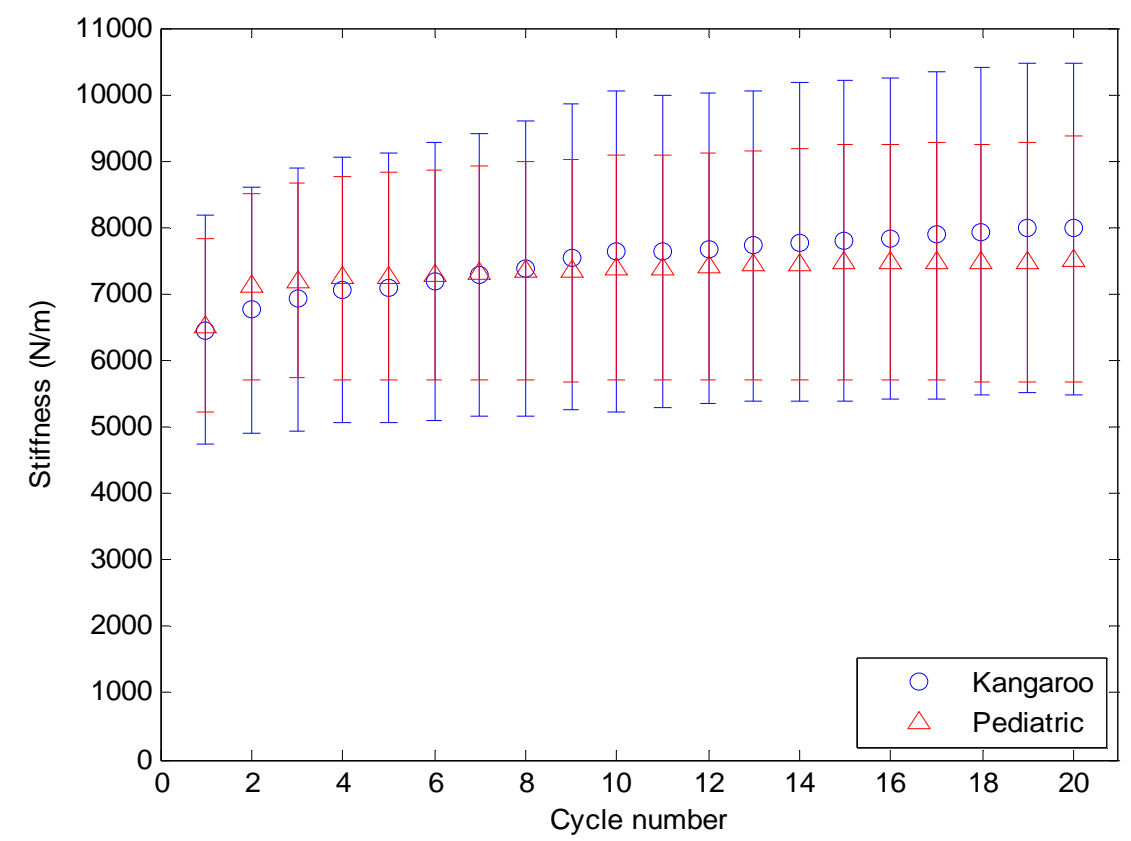

Figure 3.7: Average stiffness, k, (calculated using Equation 3.2) per cycle for juvenile kangaroo carcasses and pediatric PMHS CPR tests.

The force-deflection curves from kangaroo CPR tests were comparable to the elastic force-deflection curve fit to the lumped mass model for the youngest clinical pediatric patient (8-years-old) performed by Maltese et al. (2008). Although the kangaroo chest deflections were less than the clinical patient chest deflections, the kangaroos had approximately $50 \mathrm{~N}$ at a deflection of $10 \mathrm{~mm}$, which fit the model curve and followed the trend along the mean elastic force from the pediatric patient data (Figure 3.8, right). The PMHS (6- and 7-year-olds) force-deflection responses are also comparable to the 8-year-old patient (Figure 3.8, left). The 9-year-old clinical patient was less stiff compared to the pediatric PMHS and kangaroo subjects (Figure 3.9). 

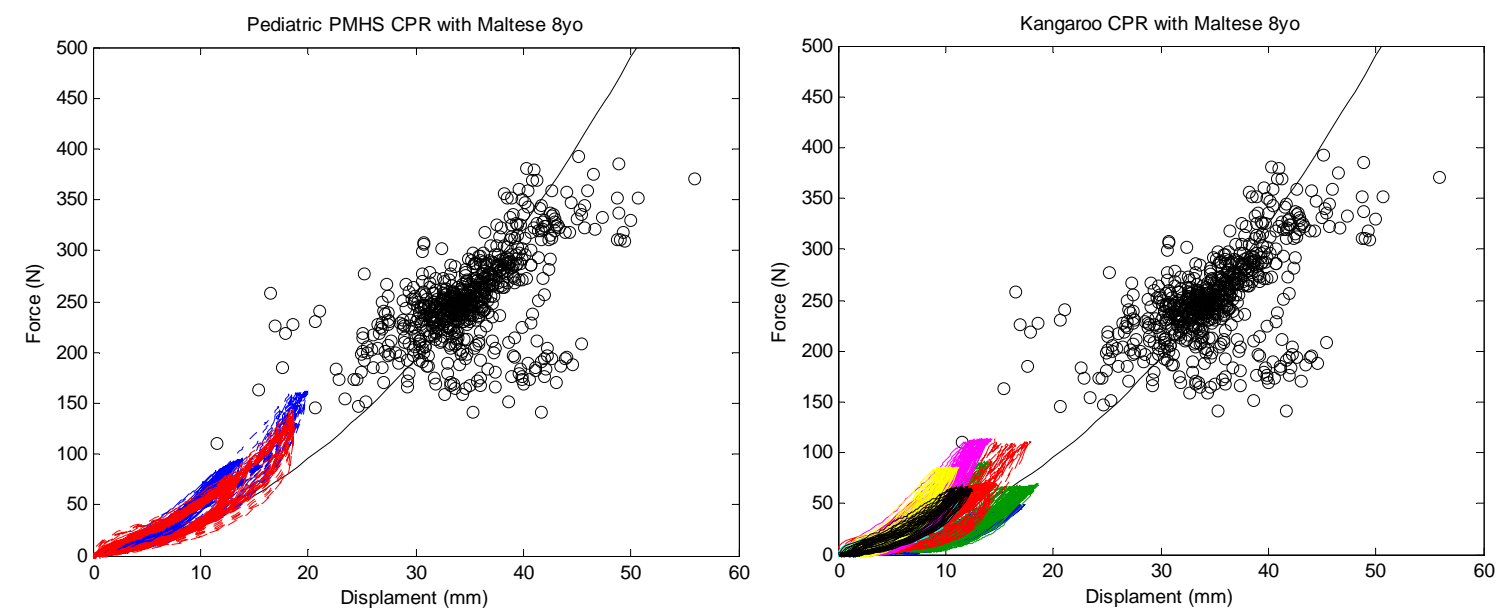

Figure 3.8: Pediatric (left) and kangaroo (right) CPR force-deflection curves plotted on top of max deflection points and elastic force curve from model for 8-year-old patient.
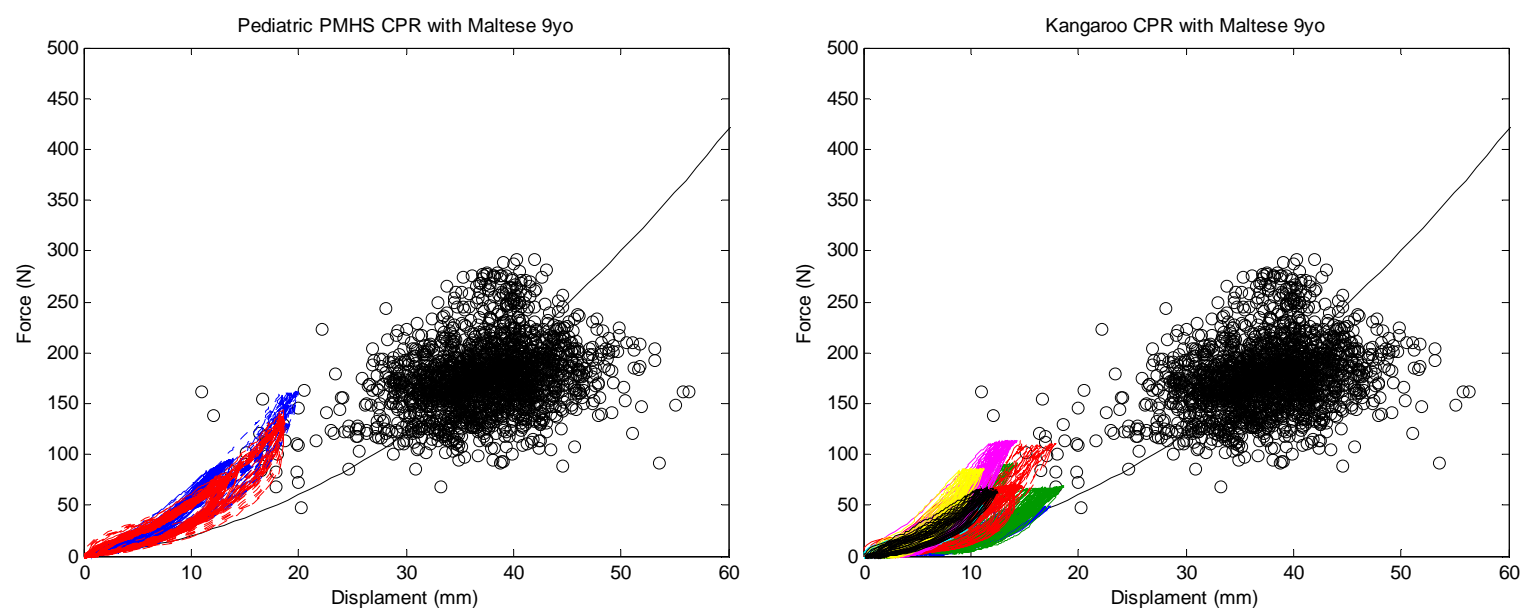

Figure 3.9: Pediatric (left) and kangaroo (right) CPR force-deflection curves plotted on top of max deflection points and elastic force curve from model for 9-year-old patient.

\section{5: Discussion}

\subsection{1: $\quad$ Experiments}

The placement of the puck on the kangaroo sternum was less stable compared to the human due to the conical shaped torso of the kangaroo, with a circular axial cross-section, compared to the more reniform axial cross-section of a human's torso. The sensor placement is a possible cause of the molding effect seen in the kangaroos, which is observed as the stiffness increases as the cycle number increases (Figure 3.7). 
The conical shape of the kangaroo thorax resulted in loading the sternum at an angle orthogonal to the posterior spine but not orthogonal to the sternum. In general, when a pediatric human is laying supine on a flat surface, the sternum is basically parallel to the spine (Figure 2.5). The kangaroo thoracic force vector was measured orthogonal to the sternum, while the deflection was orthogonal to the spine, resulting in a less than $10 \%$ error in the force which would produce slightly different force deflection characteristics, but not enough to make a significant difference. A shim placed underneath the spine reducing sternal angle might have provided a force vector loading more similar to the puck loading vector on pediatric subjects.

The kangaroo subjects had a narrow shoulder breadth, so the puck covered approximately half of the anterior thoracic surface area. The FDS puck loaded more of the kangaroo ribs compared to the pediatric PMHS where the FDS puck area covered mostly the costal cartilage.

Differences in geometric similitude are apparent in the kangaroo CPR tests, particularly the conical shape of the kangaroo. The conical shape of the thorax could lead to difficulties in belt positioning for the sled tests.

\subsection{2: $\quad$ Data Analysis}

Elastic force at $10 \%$ of chest deflection was used to compare the kangaroo and pediatric PMHS subjects, while Kent et al. (2012) compared elastic forces at $15 \%$ chest depth compression of the pediatric patients to the pediatric PMHS subjects, and reported that the pediatric PMHS subjects were stiffer than for the 8- and 9-year-old patients. Extrapolation of the kangaroo tests to $15 \%$ chest depth would not be very 
accurate since all of the kangaroo compressions were $10 \%$ of chest depth or less. The two youngest pediatric CPR patients were used for comparison even though they were slightly older than the target age group of 6-years-old. Additionally, the mass of the 8year-old patient was $40 \mathrm{~kg}\left(100^{\text {th }}\right.$ percentile for body mass) and the 9-year-old patient was $17 \mathrm{~kg}$ ( 0.2 percentile body mass). The body mass contributed more to the stiffness trend for the two youngest clinical patients than the age of the subjects. The pediatric PMHS were stiffer than pediatric patients, and the kangaroo carcasses were comparable to the pediatric PMHS and therefore stiffer than the pediatric patients. Physiological difference between living and non-living subjects may have contributed to the noted differences.

The pediatric patients had a large number of data points from each cycle and there was a large spread of those data points as well as larger chest deflection inputs. There were repeated tests on a small number of pediatric subjects and kangaroo carcasses, but there was a larger range of sizes for the kangaroo subjects (17-36kg).

The simple lumped mass model may not be the best model to characterize the thoracic CPR loading. This model was chosen to replicate the existing pediatric CPR analysis by Maltese et al. (2008) for comparison. A non-physical artifact of the model produces negative dampening values (Appendix B, Table B1). The model would have been better if constraints were placed on the model when fitting the data.

Due to the large amount of molding observed in the kangaroo thoracic response, zeroing the data was needed to be able to characterize each compression cycle for comparison. Zeroing the deflection for each cycle to calculate stiffness and damping 
coefficients resulted in the observed increasing stiffness per cycle number; however, linear stiffness calculations using peak force and deflection values resulted in decreasing stiffness per cycle. There were no pre-conditioning tests prior to the CPR tests, and only 20 cycles per test. A larger number of cycles per test might have reduced the molding effects observed.

Overall the stiffness in this kangaroo animal model was quantitatively similar to the pediatric human under this specific CPR loading condition; however, this condition is not representative of the loading condition seen in automotive crashes. The area of the thorax loaded is mainly the sternum while a shoulder belt also loads the clavicle and lower ribs more laterally. The rate of sternum displacement is an order of magnitude lower than that observed in crashes resulting in a damping force that is almost negligible. The set of experimental tests described in the next chapter has a loading rate more representative of an actual vehicle crash.

\section{6: Summary}

This study evaluated the response of seven juvenile kangaroo carcasses under a cyclic CPR loading to the mid sternum. The kangaroo thoracic responses were compared to CPR data from two pediatric PMHS (i.e. a 6-year-old and a 7-year-old) and data from clinical patients (i.e. a 8-year-old and a 9-year-old). These juvenile kangaroos exhibited thoracic elastic stiffness similar to the pediatric human under this CPR loading condition. The next chapter expands the biomechanical evaluation by considering a higher-rate loading environment. 


\section{Chapter 4: Hub Impact Loading}

\section{1: Introduction and Goals}

This chapter evaluates the thoracic response of three juvenile kangaroo carcasses under blunt hub impact loading, replicating the test-setup reported by Ouyang et al. (2006) (Ouyang tests) on pediatric PMHS. The pediatric PMHS in the Ouyang tests were separated into an old cohort and a young cohort, which had different impacting masses and geometries. The kangaroo comparison focused on the old cohort, which consisted of four subjects between the ages of 5 and 7.5 years-old and one 12-year-old. The kangaroo data were compared to data from Parent et al. (2010) who reanalyzed the data from the original Ouyang tests. The hub impact tests allow the subject to have a free-back boundary condition, and a loading rate more representative of a vehicle crash, unlike the CPR tests described in the last chapter.

\section{2: Experimental Procedures and Data Analysis}

\subsection{1: $\quad$ Overview and Test Matrix}

After subjects K08M, K09F and K10M were tested in non-injurious CPR cyclic loading, they were subjected to blunt hub impacts. Each kangaroo was impacted anteriorly at two different levels (Figure 4.1 and Figure 4.6): first, the hub centered anteriorly in the transverse plane from the fourth thoracic vertebrae (T4) and second, the bottom edge of the hub positioned just superior of the xiphoid process (XP). The inferior impact occurred subsequently after palpating the subjects to check for fractures or loss of structural integrity. After the second impact, the kangaroos were palpated again, and a full body necropsy was performed to document and describe any injuries 
generated during testing. The test matrix and the chest depth at level of impact are reported in Table 4.1

The kangaroo hub tests were designed to replicate the Ouyang set-up for the old cohort subjects (Table 4.2 ) by using a $3.5 \mathrm{~kg}$ circular blunt hub with a $75 \mathrm{~mm}$ diameter impacting surface, traveling at a nominal speed of $6 \mathrm{~m} / \mathrm{s}$. Experimental differences in the current test set-up in relation to the Ouyang tests included a three-dimensional motion tracking system (Vicon) instead of a chest band to measure chest deflection, differences in positioning due to thorax geometry and anatomical features, and a distinct free-flight portion of the impacting stroke.

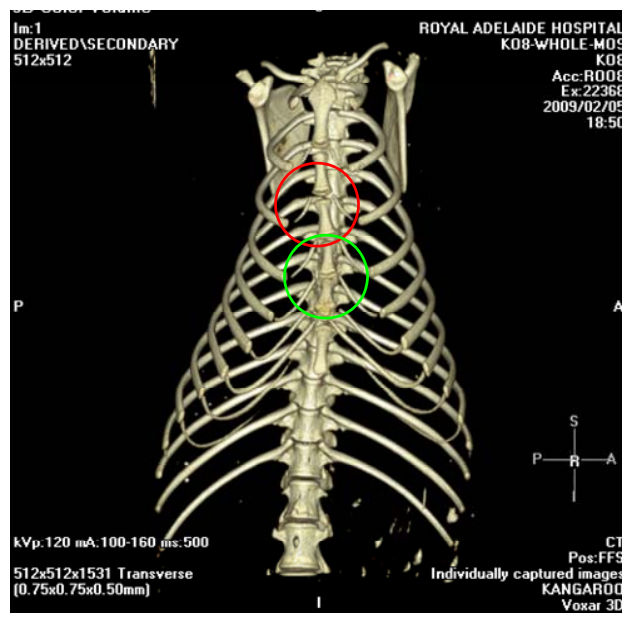

Figure 4.1: Outline of hub impact locations (red outline-T4 impact, green outline-XP impact) on CT of kangaroo thorax (K08M)

Table 4.1: Kangaroo Hub Test Matrix and depths at impact levels

\begin{tabular}{|c|c|c|c|c|}
\hline Test ID & Subject & $\begin{array}{l}\text { Whole Body } \\
\text { Mass (kg) }\end{array}$ & $\begin{array}{c}\text { Chest depth at } \\
\text { impact level }(\mathrm{mm})\end{array}$ & $\begin{array}{c}\text { Anterior Impact } \\
\text { Level }\end{array}$ \\
\hline K08M_T4 & K08M & 33 & 165 & Across from T4 \\
\hline K08M_XP & K08M & 33 & 175 & Above XP \\
\hline K09F_T4 & K09F & 17 & 110 & Across from T4 \\
\hline K09F_XP & K09F & 17 & 130 & Above XP \\
\hline K10M_T4 & $\mathrm{K} 10 \mathrm{M}$ & 23 & 100 & Across from $\mathrm{T} 4$ \\
\hline K10M_XP & K10M & 23 & 140 & Above XP \\
\hline \multicolumn{2}{|c|}{ Average } & $24 \pm 8$ & $137 \pm 30$ & \\
\hline
\end{tabular}


Table 4.2: Pediatric PMHS subjects used in hub tests by Ouyang et al. (2006)

\begin{tabular}{|c|c|c|c|c|}
\hline Subject & Age (Years) & Sex & Mass (kg) & Stature (m) \\
\hline \multicolumn{5}{|c|}{ Young Cohort } \\
\hline 1 & 2 & F & 13.0 & 0.970 \\
\hline 2 & 2.5 & M & 10.5 & 0.875 \\
\hline 3 & 3 & F & 10.5 & 0.850 \\
\hline 4 & 3 & M & 13.5 & 0.930 \\
\hline Average & $2.6 \pm 0.5$ & \multicolumn{5}{|c|}{$11.9 \pm 1.6$} & $0.906 \pm 0.054$ \\
\hline \multicolumn{5}{|c|}{ Old Cohort } \\
\hline 5 & 5 & M & 13 & 1.010 \\
\hline 6 & 6 & M & 16.5 & 1.080 \\
\hline 7 & 6 & M & 20 & 1.090 \\
\hline 8 & 7.5 & F & 17 & 1.170 \\
\hline 9 & 12 & F & 29 & 1.425 \\
\hline Average & $7.3 \pm 2.8$ & & $19.1 \pm 6.1$ & $1.155 \pm 0.161$ \\
\hline
\end{tabular}

\subsection{2: $\quad$ Equipment}

A pneumatic biomechanic linear impactor (VIA Systems, Salinas, CA), rigidly secured to the ground, was used to drive the impacting mass, i.e. transfer piston, that impacted each subject. The impactor probe provided a power stroke to the transfer piston and when the impactor stopped, it released the transfer piston into free flight upon impacting the subject anteriorly. A schematic of the kangaroo test set-up is shown in Figure 4.2. 


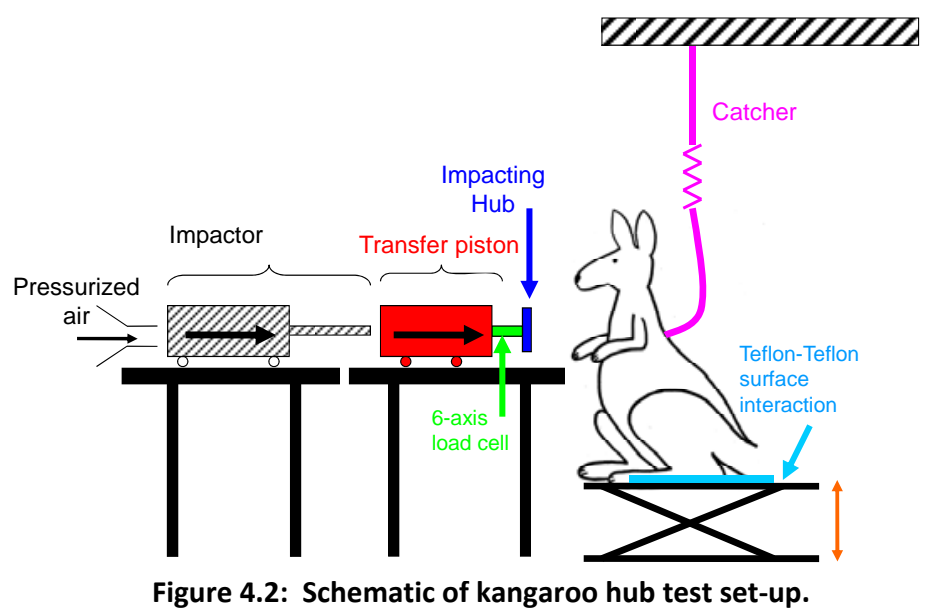

Alterations on the linear impactor mass and stroke were performed to accommodate the test design requirements for a velocity of $6 \mathrm{~m} / \mathrm{s}$. Preliminary tests were run to determine a curve fit for the velocity as a function of tank pressure in the pneumatic impactor system.

The transfer piston (Figure 4.3) traveled on linear roller bearings on the transfer piston stand which was fully decoupled from the impactor frame. A 75mm diameter hub disc was attached to a 6-axis load cell (Denton, Rochester Hills, MI) at the end of the transfer piston. The total mass of the transfer piston system was $3.5 \mathrm{~kg}$. Two accelerometers were attached to the transfer piston, one in front of the load cell, and one behind the load cell. The position of the transfer piston was measured using a linear magnetic transducer (Novotechnik, Southborough, MA). The magnet, attached to the transfer piston, hovered over the linear transducer positioned next to the bearing rail. The connection of the linear transducer to the transfer piston stand was padded with a rubber dampener to reduce noise in the linear transducer data. A backup safety bumper stop was designed to prevent the transfer piston from sliding off of the rail. 
Figure 4.3 shows details of the transfer piston and hub and specifications on the equipment used is provided in Table 4.3.

Table 4.3: Equipment Specifications-Hub Impact System

\begin{tabular}{|c|c|}
\hline \multicolumn{2}{|l|}{ Impactor } \\
\hline Type: & Pneumatic biomechanic linear impactor (VIA Systems, Salinas, CA) \\
\hline Description: & Pneumatic driven propulsion system with a probe that provides a finite power stroke \\
\hline Specifications: & $\begin{array}{l}\text { Tank max pressure: } 200 \mathrm{psi}(1.37 \mathrm{MPa}) \\
\text { Energy: } 100 \mathrm{lbs} \text { at } 44 \mathrm{ft} / \mathrm{sec}(45 \mathrm{~kg} \text { at } 13.4 \mathrm{~m} / \mathrm{s})\end{array}$ \\
\hline \multicolumn{2}{|c|}{ Position Transducer } \\
\hline Type: & Novotechnik Position Transducer Model TLM (Novotechnik, Southborough, MA) \\
\hline Description: & $\begin{array}{l}\text { Position transducer was mounted to the transfer piston frame with the passive } \\
\text { position marker magnet attached to the transfer piston. }\end{array}$ \\
\hline Specifications: & $\begin{array}{l}\text { Length: } 950 \mathrm{~mm} \\
\text { Electrical Interface: Analog } \\
\text { Supply voltage: } 24 \mathrm{VDC} \pm 20 \% \\
\text { Voltage output: }-10 \mathrm{VDC},+10 \mathrm{VDC}\end{array}$ \\
\hline \multicolumn{2}{|l|}{ Load Cell } \\
\hline Type: & Six-axis Implantable Femur Load Cell Model 6166JI4 (Denton, Rochester Hills, MI) \\
\hline Description: & $\begin{array}{l}\text { Measures three axes of forces, two bending moments, and one torsional moment of } \\
\text { the impacting hub. }\end{array}$ \\
\hline Specifications & $\begin{array}{l}\text { Capacity Fx and Fy: } 1200 \text { lbs. (5338 N) } \\
\text { Capacity Fz: } 3000 \text { lbs. (13344 N) } \\
\text { Capacity Mx and My: } 3600 \text { lb.-in. (407 N-m) } \\
\text { Capacity Mz: } 1000 \text { lb.-in. (113 N-m) }\end{array}$ \\
\hline \multicolumn{2}{|c|}{ Accelerometers on transfer piston } \\
\hline Type: & Endevco model 7264B-500 \\
\hline Description: & Placed on transfer piston to measure acceleration along the track. \\
\hline Specifications & $\begin{array}{l}\text { Range: } 500 \mathrm{~g} \\
\text { Sensitivity: } 0.8 \mathrm{mV} / \mathrm{g} \\
\text { Frequency: } 0-3000 \mathrm{~Hz}\end{array}$ \\
\hline
\end{tabular}




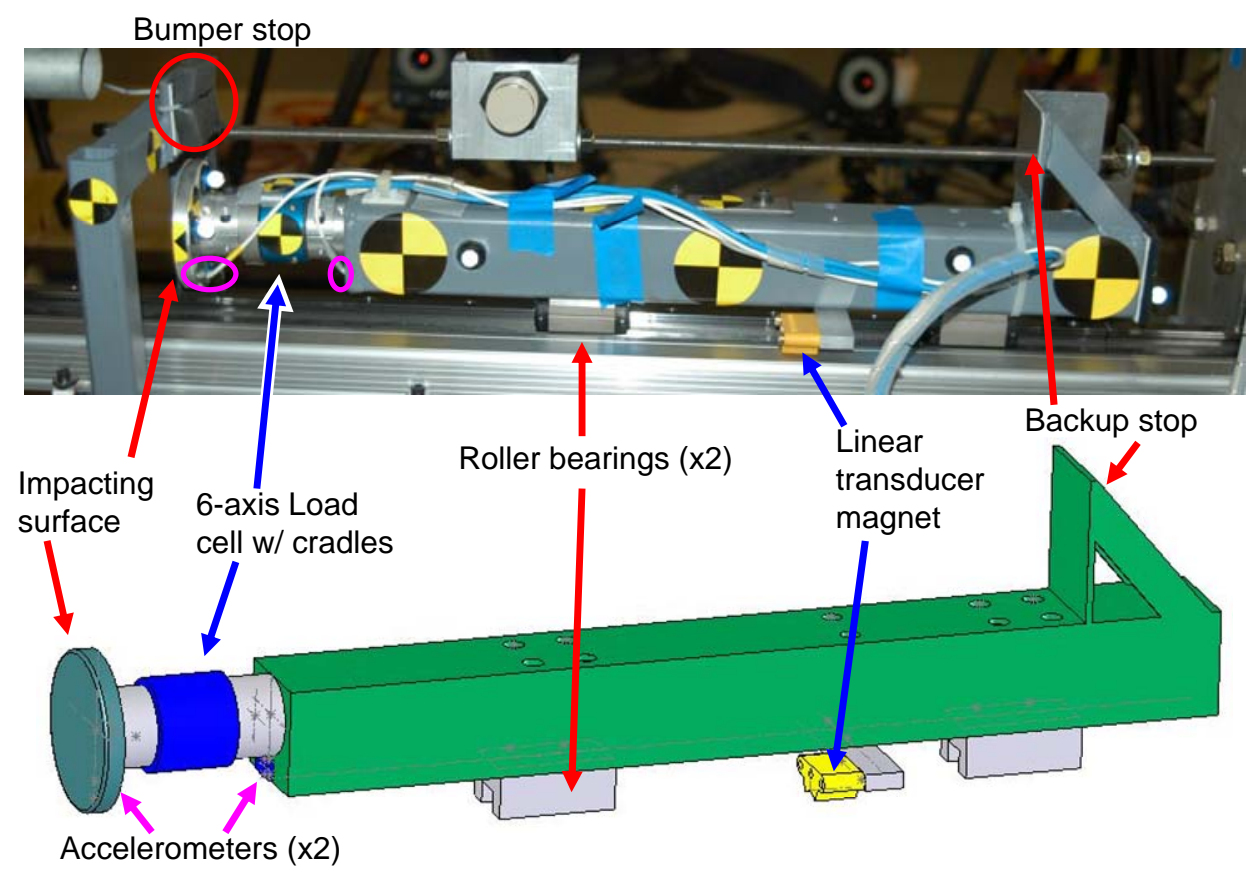

Figure 4.3: Details of transfer piston with impacting hub surface

\subsection{3: $\quad$ Subject Preparation and Positioning}

The hub test series used kangaroo subjects K08M, K09F, and K10M. These subjects were chosen to have a range of subject sizes (Table 4.1). None of these subjects had intact heads, because subjects with intact heads were saved for sled tests. Initially, subjects were thawed at room temperature, sheared, and subjected to noninjurious CPR loading prior to installing instrumentation mounts.

The subjects were prepared by installing accelerometer and Vicon marker mounts to the posterior spine and pelvis (Figure 4.4). Triaxial accelerometer cubes (Endevco model 7264B-2000) and Vicon marker arrays were attached to the mounts after seating the subject. The mount position was documented via a pre-test CT scan allowing transposition of marker location above the skin surface to the bony structure. To characterize thoracic motion, single Vicon markers were sutured to the anterior 
ribcage bilaterally on ribs 4,6 , and 8 . The markers were sutured to the rib cage by looping the suture around each specific rib. Single Vicon markers were also superficially sutured to each humeral head. A plate was screwed into the manubrium and used to attach a single axis accelerometer and a single Vicon marker. Figure 4.5 shows the location of all Vicon single markers and arrays.

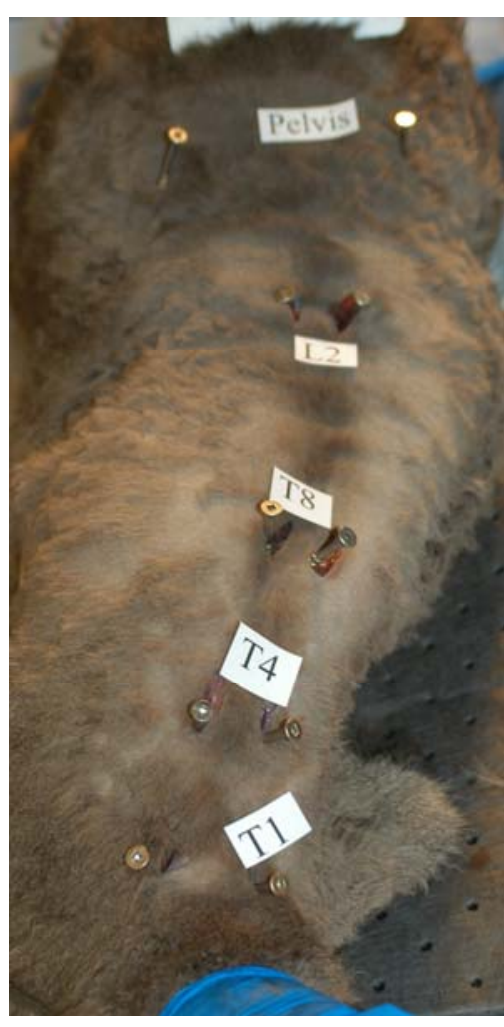

(A)

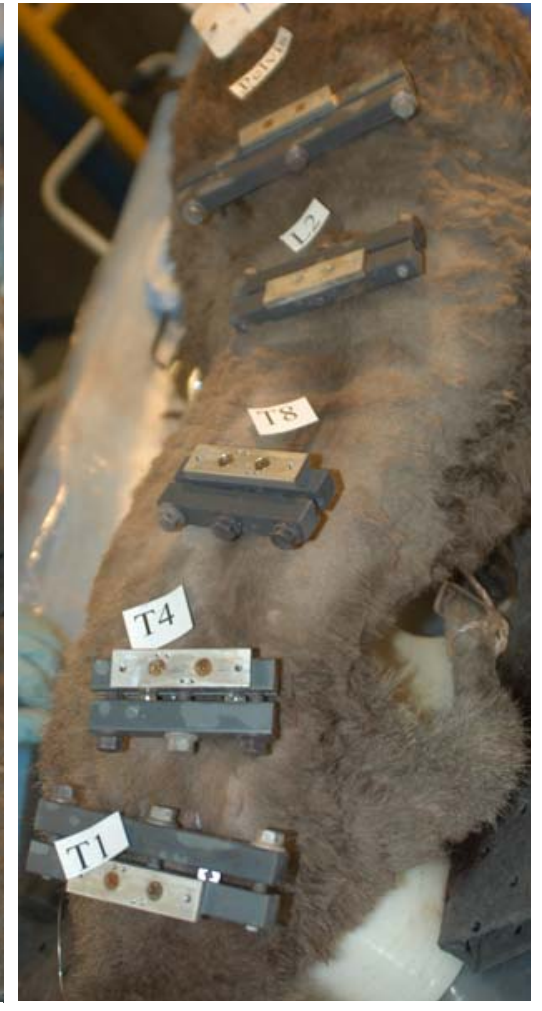

(B)

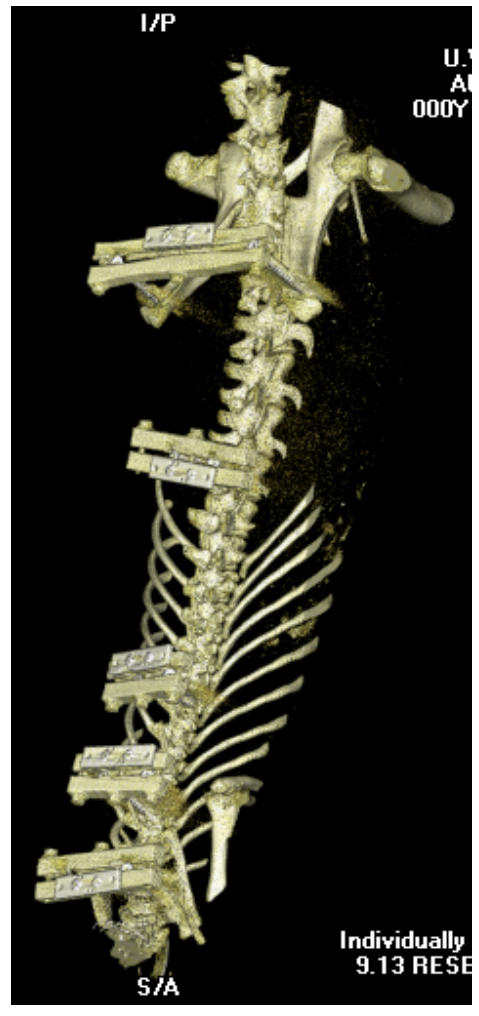

(C)

Figure 4.4: Spinal mounting method-Wood screws $(A)$ were screwed into the lateral spinous processes of the target vertebra with the objective being to maximize the cortical bone through which it passed. A two piece radiotranslucent plastic assembly (B) clamped to the heads of the screws forming the base for the accelerometer and marker plates (C) CT image of the mounts rigidly attached to vertebral bodies and pelvis.

An endotracheal tube (ETT) was installed for the lungs to be inflated during impact. The ETT was positioned such that the cuff was inflated just superior to the bifurcation of the trachea. Each subject had a continuous inflation of the lungs throughout the test at 1 psi. 


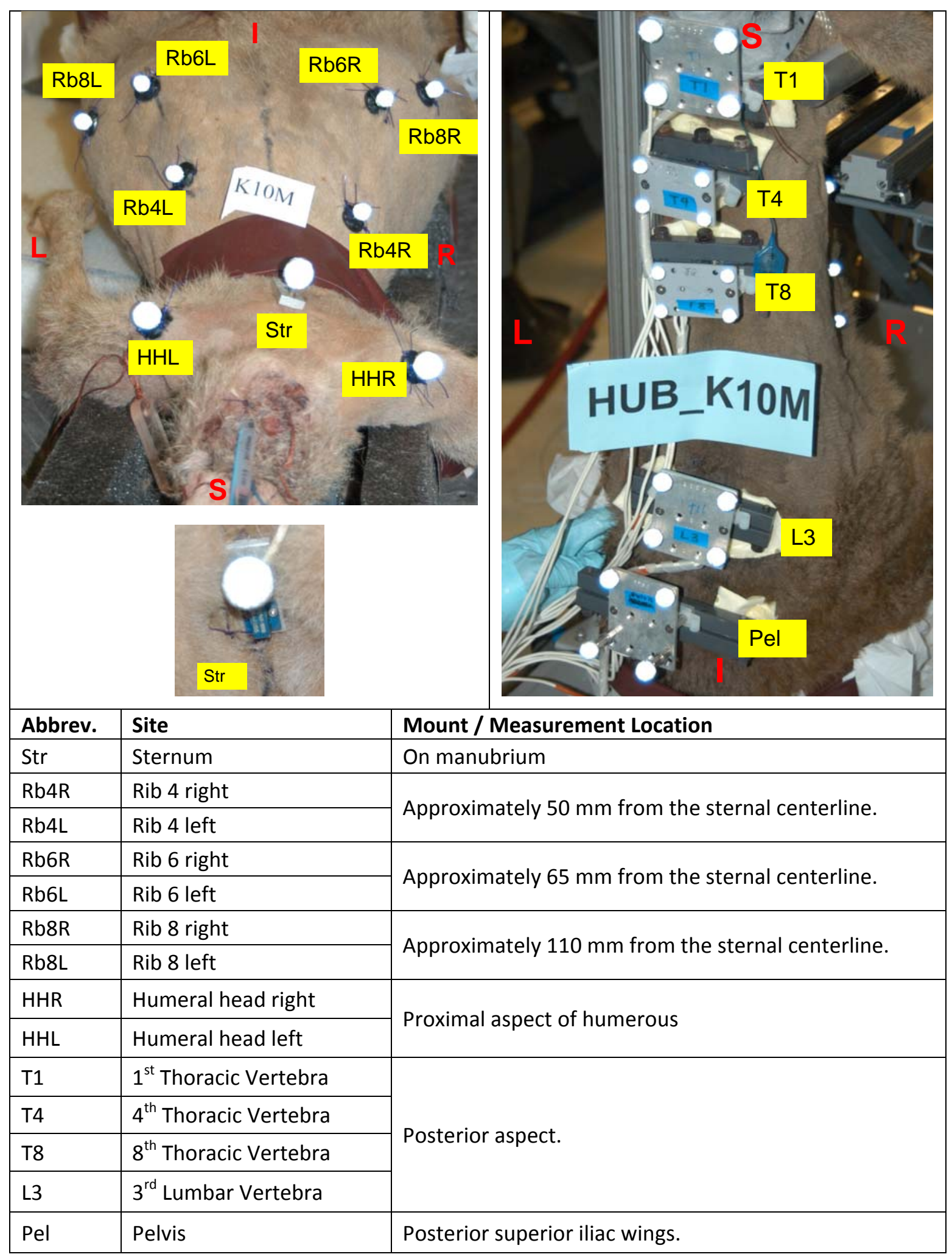

Figure 4.5: Vicon marker plate arrays and single marker locations on kangaroo hub test subject 
The description of test equipment and hardware for positioning are shown in Figure 4.7 with descriptions in Table 4.4. Each subject was seated on a level rigid surface of a hydraulic lift table that allowed vertical height adjustment with respect to the hub. The subject was seated on a piece of foam $(375 \mathrm{~mm} \times 210 \mathrm{~mm} \times 13 \mathrm{~mm})$ to create a Teflon-Teflon interaction for the seated surface. The Teflon-Teflon interface had a static coefficient of friction of 0.256 and a dynamic coefficient of friction of 0.239 . Each subject's tail was removed, and the lower extremities available were tied together and bundled against the pelvis with a diaper. Foam spacers were placed underneath the pelvis to aid in positioning.

The subjects were positioned on the hydraulic lift table facing the impactor such that the impact location was centered on the sternum, and the sternum was ideally parallel to the hub face (Figure 4.6). These were the two main positioning criteria for the Ouyang tests. The upper body was supported by rods, parallel to the load path, underneath the arms in the axillary region (i.e. under the armpits). The arm supports, lined with Teflon, were positioned anterior to the subject to allow the subject to slide off the rods posteriorly at impact. The kangaroo arm supports had a smaller contact area compared to the Ouyang subjects supported underneath the length of the arm. A rope, in series with an elastic cord that was suspended from a crane, was looped around the subject's shoulders as a catcher, post impact. 

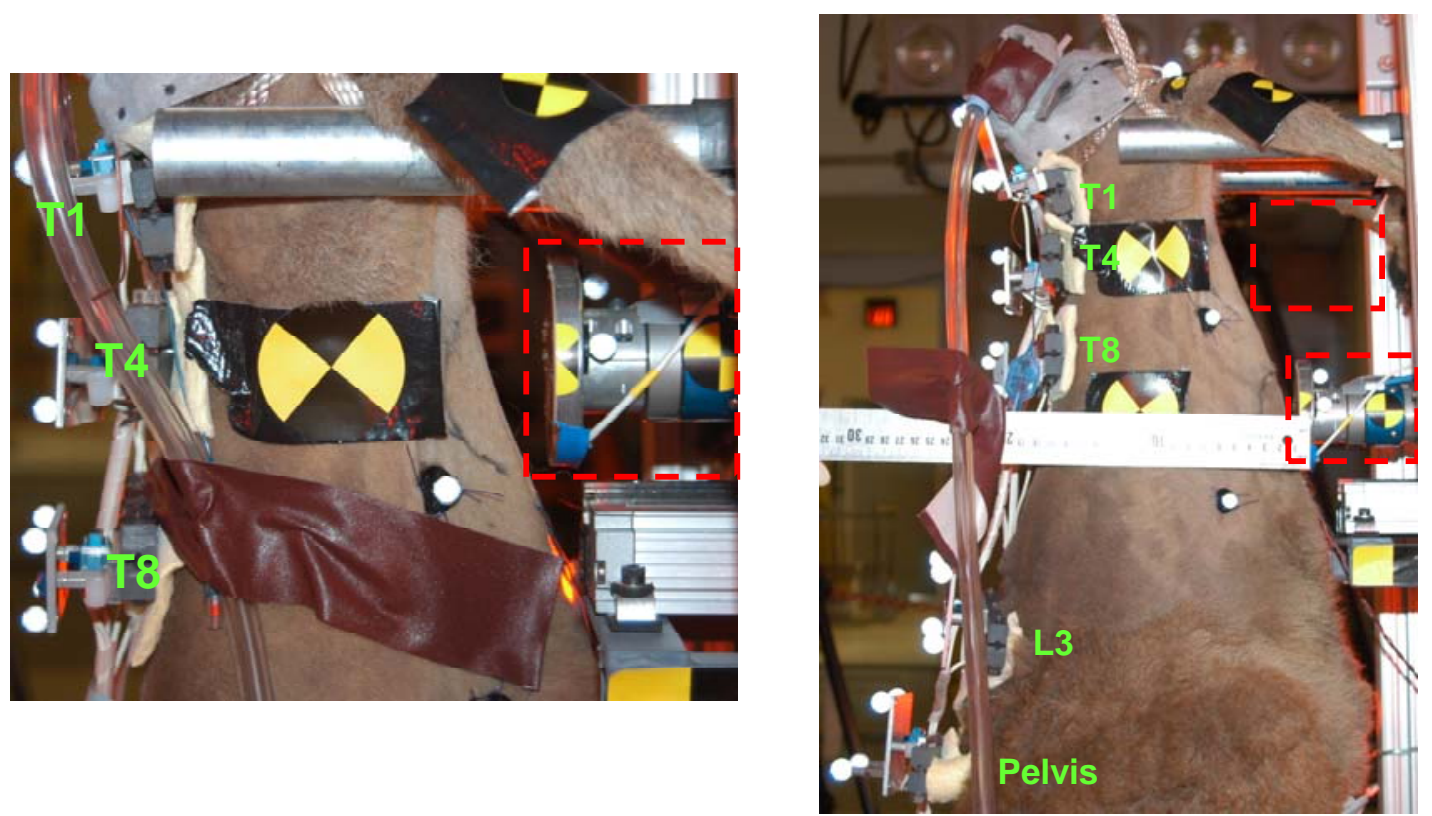

Figure 4.6: Sagittal view of T4 impact level (left) and XP impact level (right) on subject K10M positioned on arm supports 


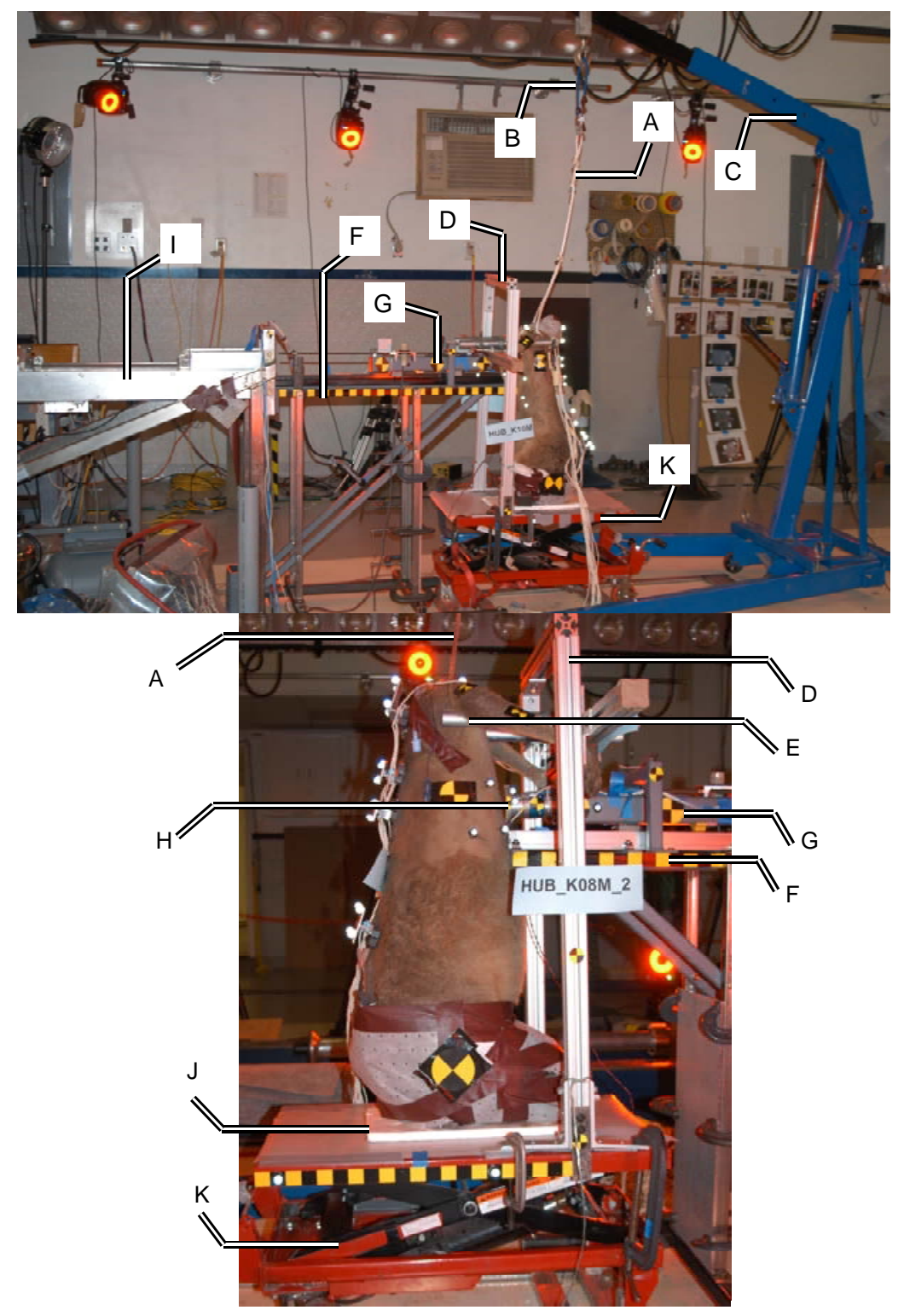

Figure 4.7: Hub Impact Test Hardware with labels defined in Table 4.4

Table 4.4: Hub Impact Test Hardware Descriptions

\begin{tabular}{|l|l|l|}
\hline A & rope tether & loosely attached to serve as a catcher post impact \\
\hline B & elastic cord & allow compliance in tether when catching \\
\hline C & crane & used to suspend catcher \\
\hline D & arm support fixture & adjustable frame to position arm supports vertically and laterally for each subject \\
\hline E & arm supports & position and support subject pre-impact \\
\hline F & transfer piston stand & rigidly attaches transfer piston rails to ground and is fully decoupled from the impactor \\
\hline G & transfer piston & $3.6 \mathrm{~kg}$ mass impacting subject in free-flight \\
\hline H & impacting hub face & $75 \mathrm{~mm}$ diameter impacting surface \\
\hline I & impactor & pneumatic biomechanic linear impactor \\
\hline J & Teflon interface & allow low friction interface between subject and lift table \\
\hline K & hydraulic lift table & vertically adjustable to position subject for impact \\
\hline
\end{tabular}




\subsection{4: $\quad$ Data Analysis}

The hub impacting force was processed to inertially compensate for the accelerated mass by applying the following formula:

$$
F_{\text {compensate }}=F_{\text {measured }}-m_{\text {hub }} \times \ddot{x}_{\text {hub }}
$$

Equation 4.1

where $F_{\text {measured }}$ is the compression force, $m_{\text {hub }}=0.4 \mathrm{~kg}$ is the hub mass $(0.2 \mathrm{~kg})$ and half of the load cell mass $(0.2 \mathrm{~kg})$, and $\ddot{x}_{\text {hub }}$ is the hub acceleration measured by the linear accelerometer attached to the back of the hub surface. The kangaroo thoracic force deflection curves were compared to the old (5-12 years) and young (2-4 years) cohorts of Ouyang's test data reanalyzed by Parent et al. (2010). Instrumentation data was collected at $10 \mathrm{kHz}$. It was hardware-filtered to $3000 \mathrm{~Hz}$, debiased, and filtered to SAE J211-prescribed filter classes.

The Center for Applied Biomechanics has developed a methodology using VICON $M X^{T M}$ motion capture system to obtain detailed high-speed motion of subject kinematics (Lessley et al. 2010, 2011). A system of sixteen Vicon cameras was used in these tests. The data from the motion capture camera system (Vicon data) was processed to provide 3D trajectories of the markers and marker arrays mounted to the fixture and to the subjects. For cases in which a four-marker array was used, the motion of the underlying anatomical structure was obtained through a transformation from a marker-based coordinate system to the anatomical coordinate system (Lessley et al. 2010 and 2011). This rigid body analysis yielded both 3D translation trajectories and 3axis rotation information. CT images of Vicon array mounts are provided in Appendix C. 
The Vicon data is used to determine the subject's torso movement, spinal kinematics and chest deflection. Chest deflection was determined by the displacement between the center of the impacting hub and the vertebral Vicon array that is the most directly across from it.

The kangaroo thoracic force deflection curves were compared to the old and young cohort of Ouyang's test data reanalyzed by Parent et al. (2010). These tests used a chest band to measure external deflection. The reanalysis by Parent et al. (2010) compensated for discrepancies in the Ouyang test set-up where the initial portion of the stroke may still have been powered.

\section{3: $\quad$ Results}

\subsection{1: $\quad$ Experimental Observations}

Due to the conical shape of the kangaroo thorax, two of the position criteria for the original Ouyang tests were not met. When the sternum was parallel to the impacting hub face, the spine was not vertical. Subject K10M was difficult to position such that the sternum was at an angle and the hub face contacted the inferior portion of the sternum first (Figure 4.6). Additionally, the sternum for subject K10M protruded anteriorly after positioning compared the other subjects where to the curvature of the anterior rib cage was continuous. This resulted in initially impacting a smaller surface area which was basically the sternum instead of a planar anterior rib cage. The protrusion is most likely a result of the compliant costal cartilage and the sutured rib Vicon markers constricting the skin to the ribs. 
The kangaroo torso rotated about the pelvis in the $y$-axis after each impact and there was significant thorax translation in addition to thorax. Minimal or no shear was observed between the Teflon-Teflon interaction or the kangaroo pelvis and the foam; the whole torso just rotated. No skeletal fractures or injuries were observed during post test necropsies.

\subsection{2: $\quad$ Comparison with Ouyang Data}

The kangaroo peak deflection response is comparable to Ouyang's old cohort (average age of 7.3 years), but the kangaroos generated less force (Figure 4.8). Although, the kangaroo tests were designed to replicate Ouyang's old cohort, the kangaroos' force response was more similar to Ouyang's young cohort, which had an average age of 2.6 years (Figure 4.9). The kangaroo deflection response was within the range of both Ouyang's young and old cohort.

The effect of impacting at two levels provided different responses for each kangaroo subject. Subject K08M had similar force and deflection responses for both levels. Subject K09F generated the same amount of force for both impacts but the lower impact at the XP level had more deflection. The response between the two levels for subject K10M differed in peak force and deflection. The lower XP level impact generated less force and much more deflection.

The average work done by the kangaroo deformation per impact loading and unloading cycle on the pediatric old cohort was $48.7 \pm 14.1 \mathrm{Nm}$, the pediatric young cohort was $28.2 \pm 4.3 \mathrm{Nm}$, and the kangaroo subjects was $14.2 \pm 5.6 \mathrm{Nm}$. 

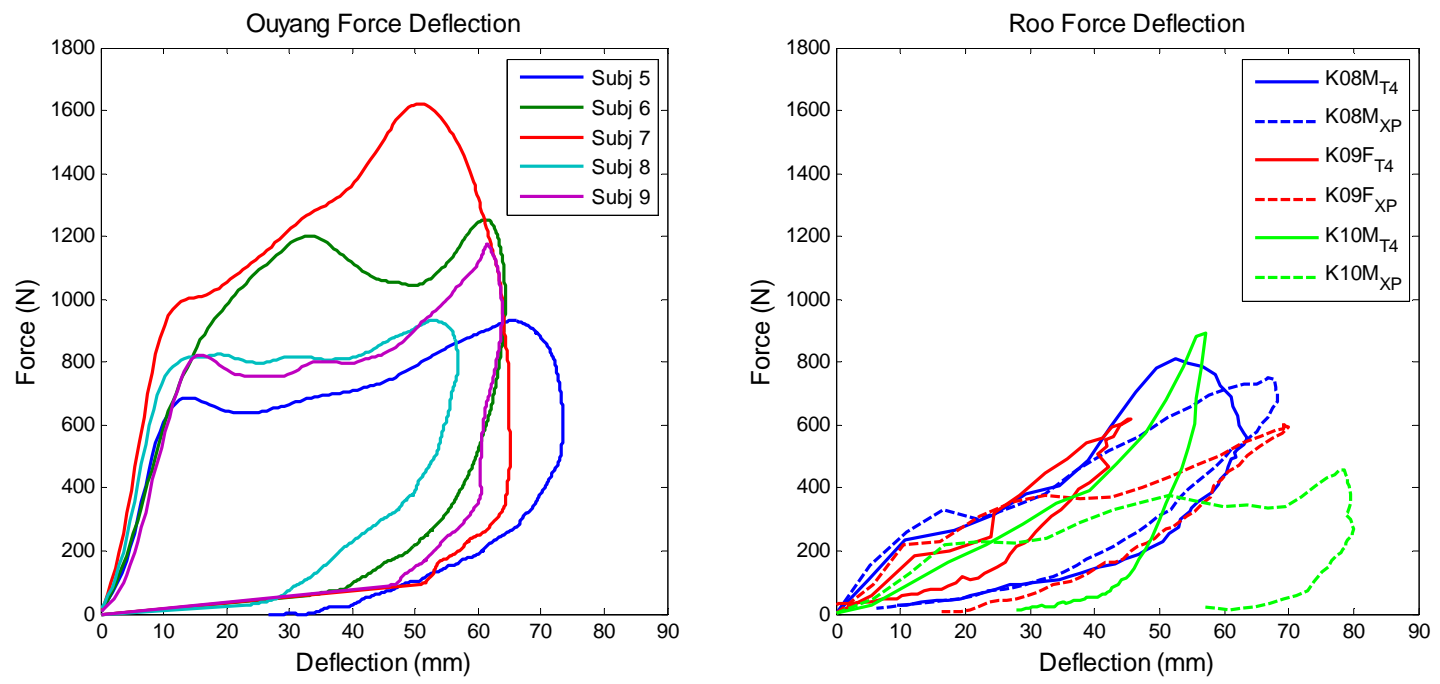

Figure 4.8: Ouyang old cohort (average age $=7.3$ years) compared to kangaroo force-deflection curves
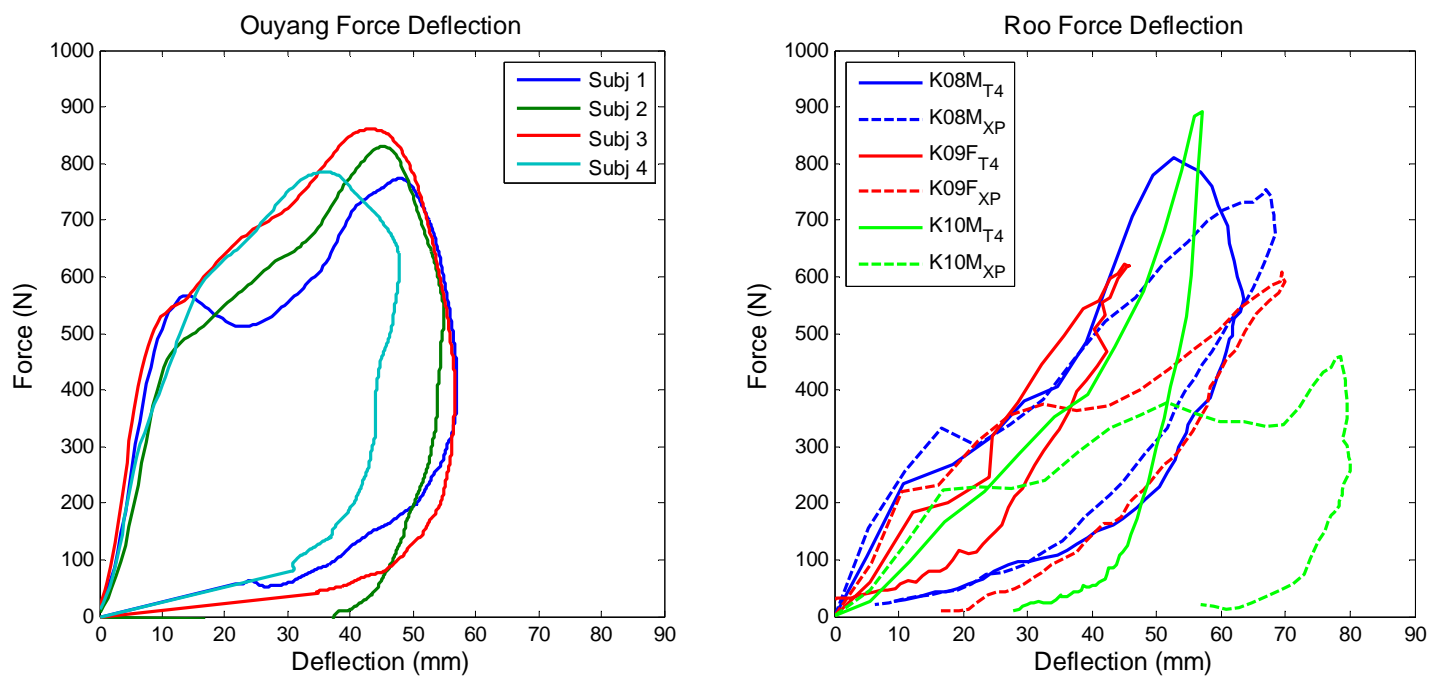

Figure 4.9: Ouyang young cohort (average age $=2.6$ years) compared to kangaroo force-deflection curves

Figure 4.10 compares the kangaroo force-deflection curves at the T4 level compared to the reanalyzed corridors developed by Parent et al. (2010). The pediatric PMHS have a large inertial contribution resulting in a force-plateau shape. 


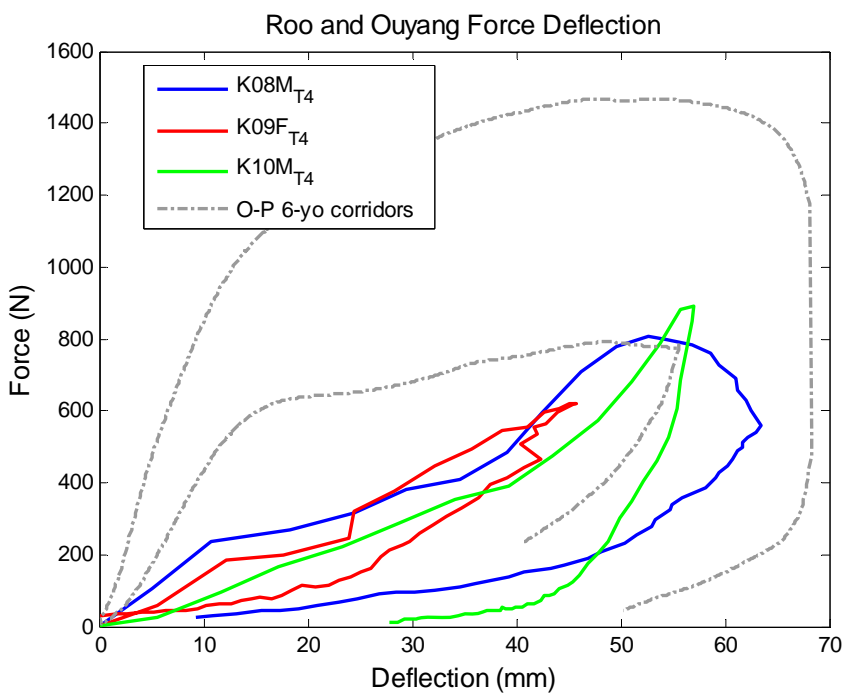

Figure 4.10: Kangaroo T4 level impact force-deflection curves compared to the corridor from data reanalyzed by Parent et al. (2010).

\section{4: Discussion}

The conical shape of the thorax presented some challenges in loading the whole sternum at initial contact of the hub. The subjects were positioned to align the sternum parallel to the impacting hub surface. When the sternum was parallel, the spinal angle with respect to the vertical was larger. The third positioning criterion for the Ouyang tests was a vertical spine, which was not met for the kangaroo tests due to the conical torso geometry.

The kangaroo thorax is much narrower superiorly compared to a human child. The conical shaped thorax provides a different mass distribution which contributed to the moment of inertia of the subject. The removal of the kangaroo's head in addition to the lower extremities wrapped against the pelvis decreased the moment of inertia, which is a main cause of the tipping motion of the kangaroo upon impact. The low friction Teflon interface for the seat was not implemented on any of the tests since the 
whole body tipped over instead of the pelvis shearing along the seat surface. The arms were only supported under the arm pits instead of the length of the arm (Ouyang tests) due to constraints of the shoulder joint range of motion as well as to minimize friction. This contributed to the large amount of upper thorax translation. A lot of the energy from the hub impact on the kangaroos was transferred to kinetic energy observed in the translation of the thorax and tipping motion, and less in the amount of work done in the thoracic deformation. Additionally, the length of the torso was significantly greater than that of a human child contributing to the tipping motion. The spinal kinematics of the kangaroo subjects were different than what would be expected in a human loaded with a blunt frontal impact.

The juvenile kangaroos appear less stiff compared to the targeted human 6-yearold. The data were closer to the young cohort (average age $=2.6$ years) in Ouyang's study, though the comparison is limited because the young cohort in Ouyang's test involved a smaller impacting mass of $2.5 \mathrm{~kg}(50 \mathrm{~mm}$ diameter $)$ instead of $3.5 \mathrm{~kg}(75 \mathrm{~mm}$ diameter) used for the older cohort. The lower level impacts on the kangaroos did not make a significant difference on the force-deflection characteristics of the thorax.

Due to the narrow thorax, this test series did not use chest bands to determine thoracic deflection; instead the Vicon motion capture system was used. The chest band used on the pediatric subjects and not on the kangaroos is a possible factor for the lower effective stiffness response. Chest bands consist of a steel band with strain gauges attached along the band. They are normally used on an adult thorax with a 
larger thoracic circumference than a child, and much larger than the juvenile kangaroo thoracic circumference.

Using the resources available, there were some differences in the test setup equipment. The Ouyang test fixture reported a $3.5 \mathrm{~kg}$ impacting mass, but there is an ambiguous distinction between the impacting mass, that never disconnects from the driving piston, compared to the impactor and free flight transfer piston in the new test fixture. The impacting probe in the Ouyang study could potentially still had been powered at the point of contact with the subject (Parent et al. 2010). The new test setup was designed such that the driving impactor stopped, allowing the transfer piston to continue in a discernible free flight phase prior to impact. There were some variations in the new test set-up regarding the power stroke compared to the original Ouyang (2006) tests, but it provided a more defined test conditions for analysis.

A large inertial spike is not observed in the kangaroo force-deflection data compared to the pediatric PMHS data. This is most likely an effect of the compliant conical-shaped rib cage and a smaller effective sternal mass of the kangaroo.

Hub impacts are not representative of the loading condition children are subjected to in automobile crashes. A shoulder belt properly positioned does not impact the thorax. As noted in the CPR tests, the hub loading geometry is also not representative of the anatomical features loaded by a shoulder belt. The next set of experimental tests has a loading rate representative of real world car crashes, as well as the loading geometry of a shoulder belt. 


\section{5: Summary}

This study evaluated the response of three juvenile kangaroo carcasses under blunt hub impact loading. Each subject was impacted anteriorly at two different levels, mid-sternum across from T4 and lower sternum where the bottom edge of the hub was at the xiphoid process. These tests were designed to replicate hub impact tests on pediatric PMHS performed by Ouyang (2006). Although there were some differences in the test set-up including: 3-D motion tracking system to determine torso movement including chest deflection instead of a chest band, differences in positioning due to thorax geometry and anatomical features, and a distinct free-flight portion of the impacting stroke.

The juvenile kangaroo appear less stiff compared to the targeted human 6-yearold, but the data was more comparable to the young cohort (average age $=2.6$ years) in Ouyang's study. The kinematics of the kangaroo torso had a large amount of translation in addition to chest deflection due to the distribution of mass of the kangaroo. This foreshadows the fact that differences in geometric similitude could potentially cause nonhuman-like response in an inertial sled test environment.

The hub tests provided more information on the kinematics of the kangaroo in a loading environment with less boundary conditions than the previous CPR tests. The next step is to test the animal model in the loading environment representative of a car crash. 


\section{Chapter 5: Sled Tests}

\section{1: Introduction and Goals}

In this chapter, the kangaroo was evaluated in an environment more representative of the in-vehicle, restrained frontal impact to determine whole body kinematics occurring in frontal impacts. The CPR tests and blunt hub impacts did not have a belt loading geometry. Additionally, the CPR loading rate is an order of magnitude too low, and the hub tests have an impact not observed in belt loading. The purpose of this study was to assess the eastern grey kangaroo in an accelerated crash simulator or sled test environment. Kangaroo sled tests have not been performed previously so this study updated test conditions throughout the experiments to expose the kangaroo to an environment most representative of what would be expected of a human six-year-old in a car crash setting.

The hope is that the kangaroo animal model in a sled test environment will help provide information to understand the thoracic response and kinematics of restrained human children in a high speed frontal impact.

\section{2: Methods}

\subsection{1: $\quad$ Overview and Test Matrix}

Four kangaroo carcasses were subjected to simulated frontal crashes at low $(9 \pm$ $1 \mathrm{~km} / \mathrm{h})$ and high $(39 \pm 1 \mathrm{~km} / \mathrm{h})$ speeds. Deceleration pulses were approximately trapezoidal, characteristic of a vehicle rigid barrier frontal crash pulse (Forman et al. 2006a, 2006b), with an average maximum acceleration of $3.2 \pm 0.2 \mathrm{~g}$ (low-speed) and $14.3 \pm 0.7 \mathrm{~g}$ (high-speed). A range of kangaroo carcass sizes was tested to explore the 
effects of subject size. The heads of six kangaroos were removed prior to acquisition, so the sled test subjects (Table 5.1) were chosen from those with heads remaining. Upon closer inspection of CT scans, only one carcass, K05M, had an intact skull base. Prior to running the sled tests, these subjects were used in the CPR loading tests. Kangaroos were palpated between subsequent tests to check for fractures or loss of structural integrity. A full body necropsy was performed at the conclusion of the sled tests.

\begin{tabular}{|c|c|c|c|c|}
\hline \multirow{2}{*}{ Table 5.1: Test matrix for sled tests } \\
\begin{tabular}{|c|c|c|c|c|}
\hline K01F* & K02F & K06F & K05M \\
\hline Low Speed & $\begin{array}{l}1433 \\
1434\end{array}$ & 1436 & 1438 & 1440 \\
\hline High Speed & 1435 & $\begin{array}{l}1437 \\
1446\end{array}$ & 1439 & 1441 \\
\hline
\end{tabular}
\end{tabular}

*The first subject was a pilot subject

Performing sled tests with a new species presented significant challenges from the outset. A series of tests were performed on a pilot subject for a preliminary assessment of belt positioning, subject positioning, instrumentation, surrogate head mass, and fixture design. Procedural modifications were then implemented throughout the tests on the other three subjects. This methods section will describe the general test set-up and subject preparation after the trial subject. This section also presents alterations in the test set up initiated from observations of previous tests.

\subsection{2: $\quad$ Equipment}

The sled configuration and data collection method used by Shaw et al. (2009) were employed for this test series. An opened-framed sled buck was used to allow line of sight of retro-reflective markers (markers) used for motion capture. The motion capture provided detailed thoracic motion and chest deflection. The kangaroo carcass 
was seated on a rigid planar seat with the torso and head supported posteriorly by an adjustable matrix of steel cables. Specifications for the sled test equipment are provided in Table 5.2. Test hardware used for the sled tests are labeled in Figure 5.3. Note that some test hardware was added to the test setup through progressive alterations throughout the tests described in section 5.2.4:.

A pelvis restraint was fabricated to serve as a perfect lap belt, restricting translational motion of the pelvis, but allowing rotation about the $\mathrm{H}$-point or acetabulum. Only the custom pelvis restraint (Figure 5.1) was used due to the geometry of the kangaroo pelvis. The kangaroo's anterior superior iliac spines (ASIS) are more posterior than the lumbar vertebrae so a lap belt would not be loading an appropriate bony structure compared to the human ASIS which is anterior to the lumbar spine (Figure 5.2). Additionally, a lap belt positioned on a kangaroo would load a lot of soft tissue, unlike a human whose iliac spines are more superficial anteriorly.

When the kangaroo subject is seated on its ischial tuberosity the extended lower extremities are parallel to the seat only if they were elevated in the z-axis. The pelvis restraint clamped the femurs along the top surface of the pelvis restraint using padded U-bolts with threaded rod extensions. The ends of the amputated tibias and fibulas were positioned abut against a surface to prevent translation along the $x$-axis. Shims consisting of various thickness aluminum plates and rubber sheets were inserted in front of the tibias to provide a tight fit.

The pelvis restraint was adjustable to accommodate different kangaroo subject sizes. The whole pelvis restraint was adjustable in the $\mathrm{x}$ direction with respect to the 
seat. Shims could be added beneath the surface plate to adjust the height. The position of the U-bolt along the $\mathrm{x}$-axis was adjustable as well as the height.
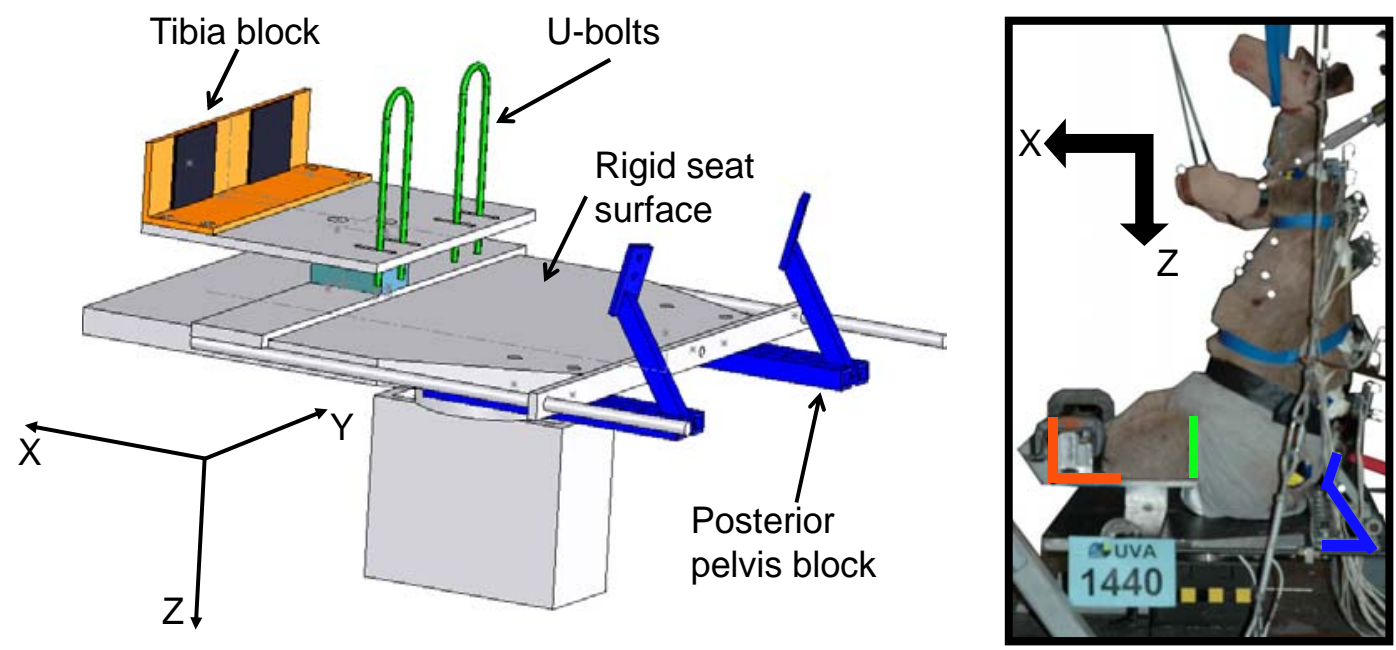

Figure 5.1: Custom designed adjustable pelvis restraint positioned on sled buck seat (left) with parts highlighted in sled set-up (right)
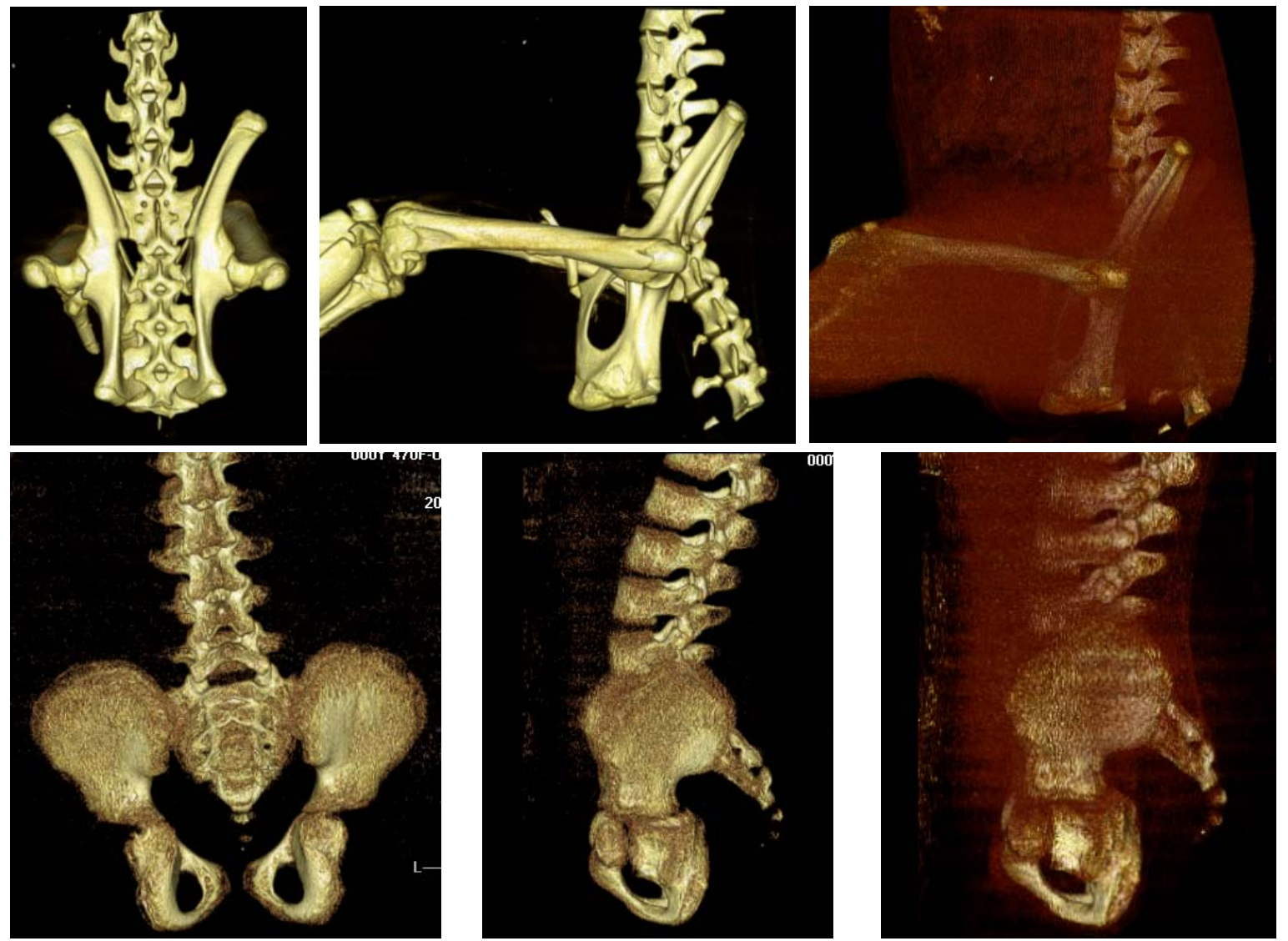

Figure 5.2: CT images of K02F pelvic region bony and soft tissue (top) and $\mathrm{CT}$ images of 7yo pelvic region bony and soft tissue (bottom) 
Table 5.2: Equipment Specifications-Sled System

\begin{tabular}{|c|c|}
\hline \multicolumn{2}{|l|}{ Crash Simulator } \\
\hline Type: & $\begin{array}{l}\text { VIA Systems deceleration test sled, model } 713 \text {, with hydraulic decelerator, model } \\
931-4000 .\end{array}$ \\
\hline Description: & Track length $100 \mathrm{ft}(30.48 \mathrm{~m})$, decelerator sled \\
\hline Instrumentation & Stationary proximity-sensor \& steel blade on sled to measure velocity \\
\hline \multicolumn{2}{|l|}{ Test Fixture (Buck) } \\
\hline Type: & Rigid platform \\
\hline Instrumentation & Front center X-axis accelerometers (2) \\
\hline \multicolumn{2}{|l|}{ Seat } \\
\hline Type: & Rigid plate \\
\hline Instrumentation & 6 channel load cell model 2531 (Denton, Rochester, MI) \\
\hline $\begin{array}{l}\text { Backrest and } \\
\text { Headrest }\end{array}$ & $\begin{array}{l}\text { UVA custom consisting of cables and wires designed to maintain the trunk and head } \\
\text { position during the time in which the buck is accelerated down the track. }\end{array}$ \\
\hline \multicolumn{2}{|l|}{ Belt Restraint } \\
\hline Type: & $\begin{array}{l}\text { Custom 2-point belt, only shoulder belt section; no retractor } \\
\text { Webbing - Narricut, International twill pattern 13195, 6-8\% elongation, } 6000 \mathrm{lbf} \\
(26.7 \mathrm{kN}) \text { minimum tensile strength (intact) } \\
\text { Hardware - Aftermarket length adjusters and anchors. } \\
\text { Custom load limiter }\end{array}$ \\
\hline Instrumentation & $\begin{array}{l}\text { Belt-tension load cells (2): Messring } 5 B C \\
\text { Shoulder belt - Near upper shoulder belt anchor and above lower shoulder belt } \\
\text { anchor. }\end{array}$ \\
\hline \multicolumn{2}{|l|}{ Pelvis Restraint } \\
\hline Type: & $\begin{array}{l}\text { UVA custom design to secure lower extremities preventing translating, while } \\
\text { allowing rotation of pelvis around } \mathrm{H} \text {-point }\end{array}$ \\
\hline Instrumentation & None, rigidly attached to seat ( 6 channel load cell) \\
\hline
\end{tabular}




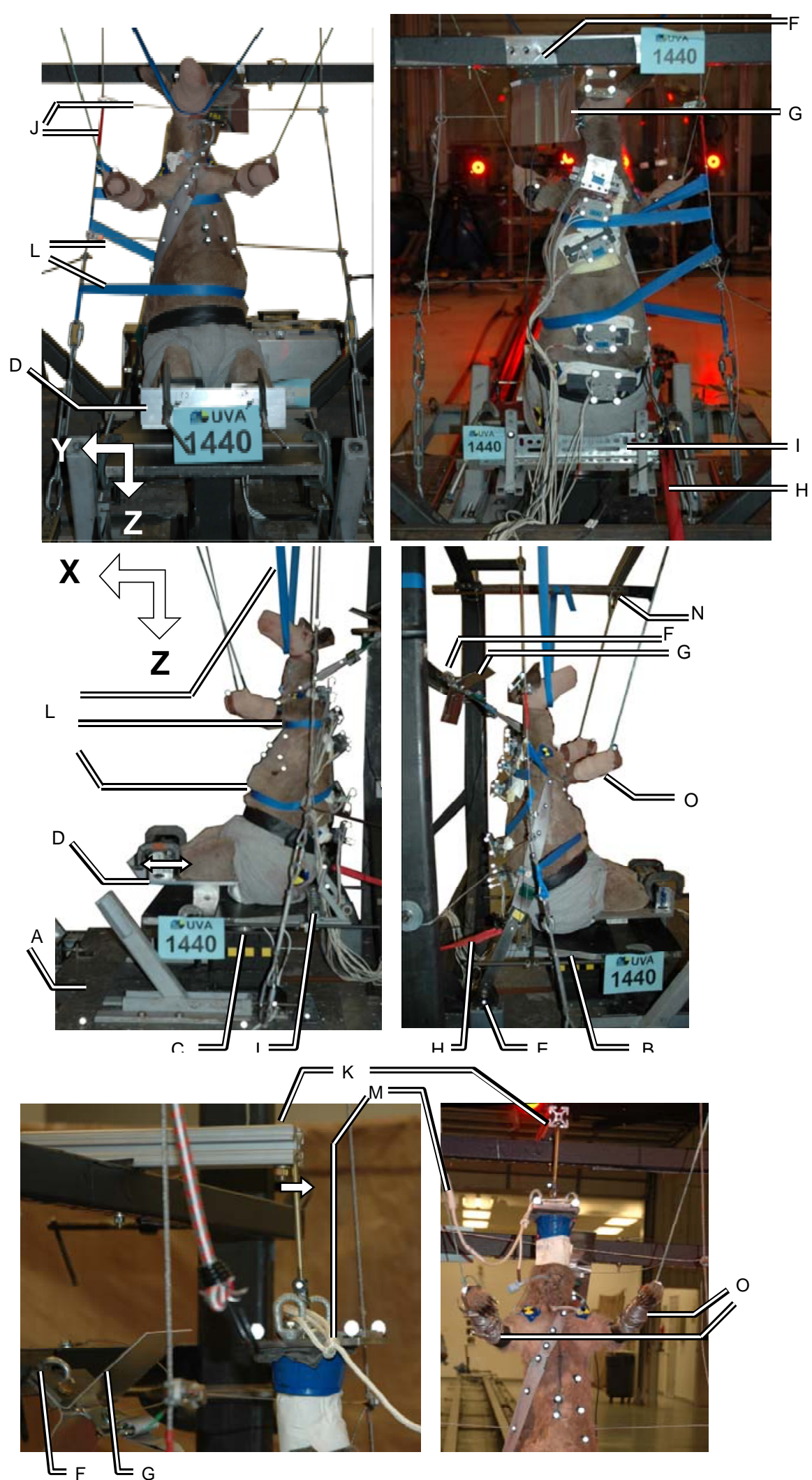

Figure 5.3: Sled test hardware. Photos of pre-test 1440 with subject K05M and 1436 with subject K02F. Label descriptions in Table $\mathbf{5 . 3}$ 
Table 5.3: Sled Test Hardware Descriptions

\begin{tabular}{|c|c|c|}
\hline A & Buck & Universal buck adaptable to various test configurations \\
\hline B & Seat & Rigid horizontal aluminum plate \\
\hline C & Seat Load cell & 6-axis load cell supporting seat \\
\hline D & Pelvis restraint & Rigidly attached to seat to restrict $X$ translation of the pelvis \\
\hline E & $\begin{array}{l}\text { Lower shoulder belt } \\
\text { anchor }\end{array}$ & Center of bolt head securing belt length adjuster tongue mount \\
\hline $\mathrm{F}$ & $\begin{array}{l}\text { Upper shoulder belt } \\
\text { anchor }\end{array}$ & Provide Y-rotation and Z rotation of belt load limiter \\
\hline G & Belt load limiter & $\begin{array}{l}\text { Two sheets of aluminum (alloy } 3003 \text { ) cut and scored to provide a } \\
\text { constant force output when torn }\end{array}$ \\
\hline $\mathrm{H}$ & $\begin{array}{l}\text { Restraint for lower } \\
\text { shoulder belt anchor }\end{array}$ & Prevent motion of lower shoulder belt anchor \\
\hline I & Pelvis block & $\begin{array}{l}\text { Originally a strap around posts and converted to an L-bracket to prevent } \\
\text { pelvic posterior migration pre-test }\end{array}$ \\
\hline $\mathrm{J}$ & Posterior support & $\begin{array}{l}\text { Adjustable back and head support provided by horizontal wires attached } \\
\text { to vertical cables }\end{array}$ \\
\hline K & Head slider bolt release & Head support for subjects with a surrogate head mass \\
\hline $\mathrm{L}$ & Head and body support & Painters tape pre-torn to hold head and torso in position pre-test \\
\hline M & Surrogate head tether & $\begin{array}{l}\text { Precautionary rope with slack to secure surrogate head mass after } \\
\text { impact }\end{array}$ \\
\hline $\mathrm{N}$ & Arm suspension & $\begin{array}{l}\text { Rope to suspend arms with mass to increase rotational moment of } \\
\text { inertia }\end{array}$ \\
\hline $\mathrm{O}$ & Arm masses & $\begin{array}{l}\text { Additional } 0.9 \mathrm{~kg} \text { lead mass secured to the distal aspect of upper } \\
\text { extremities }\end{array}$ \\
\hline
\end{tabular}

\subsection{3: $\quad$ Specimen Preparation and Positioning}

To measure thoracic and spinal kinematics, spinal mounts were installed using the same methods as hub impact subjects (section 4.2.3:), except mounts were placed in the second lumbar vertebra, $L 2$ instead of $L 3$, and the pelvis mount was positioned higher on the iliac crest so as not to interfere with the pelvis block of the seat. Single Vicon markers were sutured to the anterior ribcage after seating the subject, to deter shifting of the skin with respect to the ribs. Markers were attached only along the midline and the left side of the rib cage to prevent interference with the shoulder belt placement. The markers were sutured to the rib cage by looping the suture around specific ribs. Sutures were not looped around the sternum for the midline markers. 
Single Vicon markers were attached with tape to each upper extremity at the elbow and wrist. The location of all Vicon single markers and arrays are shown in Figure 5.4.

The head mass and moment of inertia affect the kinematics of the spine. Only one subject (K05M) had an intact skull base, so it was necessary to develop a surrogate head mass to attach to the upper cervical spine of the remaining three subjects whose skull bases were damaged during culling. The damaged skulls were removed, keeping the first cervical vertebrae (C1) intact upon which the surrogate head mass base rested. Wires were looped through the first several cervical vertebrae using the Seldinger technique. The wires were secured to screws inside a cup along with a bolt and washer. The hardware in the cup was fixed using Fastcast two-part polyurethane resin (R1 FastCast $^{\circledast}$ (GoldenWest, Cedar Ridge, CA)). Varying amounts of weight could be added to the bolt of the head mass base.

The top head mass plate consisted of an array of Vicon markers, and eyelets in which a tether rope was secured in case of failure of the head mass attachment. A slider bolt was attached to the head mass to support the subject pre-impact and released upon impact (Figure 5.3). Subject K05M, which had an intact skull base used a VICON array similar to those used on the spinal mounts where the mount was screwed directly into the superior-posterior aspect of the skull. The head for subject K05M was supported prior to impact using pre-torn masking tape.

An endotracheal tube (ETT) was installed for the lungs to be inflated with a cycle of five pumps of $0.2 \mathrm{~L}$ of air prior to each sled test, using a large syringe. The syringe pump was removed after the last inflation pump and the tube through which the air was 
delivered was left open. The ETT was positioned such that the cuff was inflated just superior to the bifurcation of the trachea.

Photo targets were placed superficially on the subject on certain bony landmarks to aid in positioning measurements and for visibility in the high speed imagers. Markers were placed bilaterally on the acromial processes, greater trochanters (the H-point was not easily palpated as seen in Figure 5.2), and the PSIS. Positioning measurements are given in Appendix D.
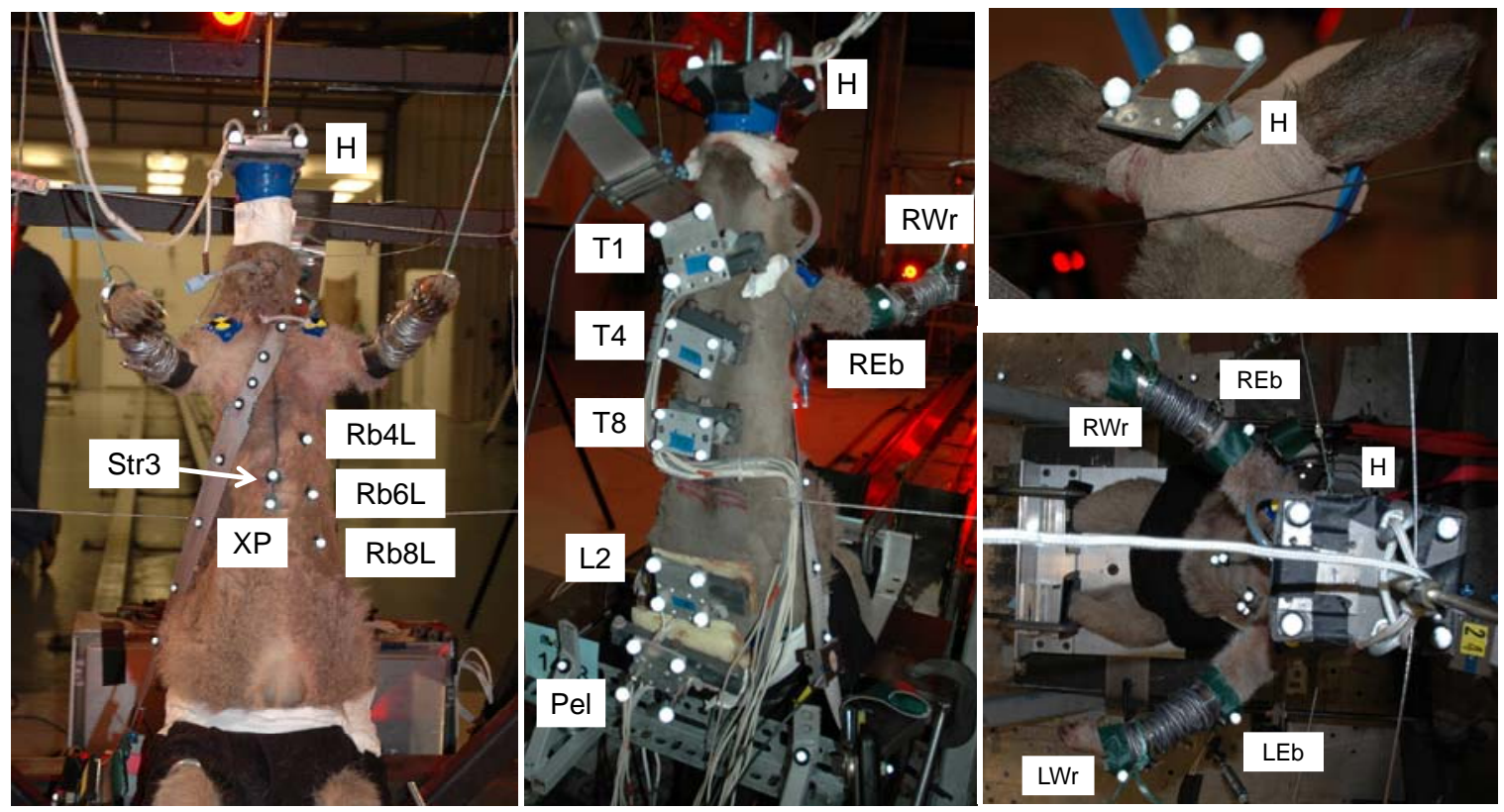

Figure 5.4: Vicon marker plates and single marker locations for K02F and K05M (head) with descriptions in Table 5.4

Table 5.4: Vicon marker and array abbreviations and locations

\begin{tabular}{|l|l|l|}
\hline Abbrev. & Site & Mount / Measurement Location \\
\hline H & Head & Approximate apex. \\
\hline LEb & Left Elbow & \multirow{2}{*}{ Distal aspect of humerous } \\
\hline REb & Right Elbow & \multirow{2}{*}{ Distal aspect of radius } \\
\hline LWr & Left Wrist & \\
\hline RWr & Right Wrist & Superior portion of 3rd sternabrae \\
\hline Str3 & Sternum & Superior portion of xiphoid process \\
\hline XP & Xiphoid Process & Approximately 30 mm from the sternal centerline. \\
\hline Rb4L & Rib 4 left & \\
\hline
\end{tabular}




\begin{tabular}{|c|c|c|}
\hline $\mathrm{Rb} 6 \mathrm{~L}$ & Rib 6 left & Approximately $50 \mathrm{~mm}$ from the sternal centerline. \\
\hline $\mathrm{Rb} 8 \mathrm{~L}$ & Rib 8 left & Approximately $75 \mathrm{~mm}$ from the sternal centerline. \\
\hline T1 & $1^{\text {st }}$ Thoracic Vertebra & \multirow{4}{*}{ Posterior aspect. } \\
\hline T4 & $4^{\text {th }}$ Thoracic Vertebra & \\
\hline T8 & $8^{\text {th }}$ Thoracic Vertebra & \\
\hline $\mathrm{L} 2$ & $2^{\text {nd }}$ Lumbar Vertebra & \\
\hline Pel & Pelvis & Posterior superior iliac wings. \\
\hline
\end{tabular}

\subsection{4: $\quad$ Pilot Subject and Test Methodology Iterations}

The first subject was used primarily as a methodology refinement tool and the experience with this subject motivated several alterations in the test set up to improve subsequent tests. Table 5.5 lists a summary of failures and observations from each sled test and a brief description of alterations performed prior to the subsequent test. The remainder of the test methodology will be described noting alterations in the test set up initiated from observations of previous tests. 
Table 5.5: Sled test observations and alterations

\begin{tabular}{|c|c|c|c|}
\hline Test \# & Subject & Test Observations & Test Alterations \\
\hline 1433 & K01F & $\begin{array}{l}\text { Belt slipped off shoulder due to large rotation in z-axis. } \\
\text { Head mass start to pull away from C-spine. }\end{array}$ & $\begin{array}{l}\text { Moved shoulder belt upper D-ring towards subject midline. Sutured a } \\
\text { cable tie looped around belt superficially anterior to the left clavicle. }\end{array}$ \\
\hline 1434 & K01F & $\begin{array}{l}\text { Shoulder belt cable tie loop sutured to skin ripped off and } \\
\text { belt slipped off shoulder. Head mass pulled out more. }\end{array}$ & $\begin{array}{l}\text { Notched shoulder belt narrower to be able to lie more medially and load } \\
\text { the just the clavicle. Attached } 0.9 \mathrm{~kg} \text { lead mass to distal aspect of both } \\
\text { upper extremities. Suspended both arms anteriorly. }\end{array}$ \\
\hline 1435 & K01F & $\begin{array}{l}\text { Arm mass and arm suspension retained shoulder belt, but } \\
\text { some thoracic VICON markers obscured. Surrogate head } \\
\text { mass detached from cervical spine. Observed large } \\
\text { amounts of lordosis at impact. }\end{array}$ & $\begin{array}{l}\text { Reduced head mass and suspended arms higher and more laterally. Cut } \\
\text { slits in skin at clavicle and routed shoulder belt cable tie loop underneath } \\
\text { skin. Installed a belt load limiter (0.063in.) to allow more forward pitch of } \\
\text { torso }\end{array}$ \\
\hline 1436 & $\mathrm{~K} 02 \mathrm{~F}$ & $\begin{array}{l}\text { Subject rotated slightly in z-axis after impact such that } \\
\text { right tibia no longer abut against shims. }\end{array}$ & \\
\hline 1437 & $\mathrm{~K} 02 \mathrm{~F}$ & $\begin{array}{l}\text { Large rotation of pelvis about y-axis and posterior } \\
\text { translation such that caudal vertebrae rested on top of } \\
\text { pelvis block strap instead of seat surface post-test. } \\
\text { Minimal engagement of load limiter. }\end{array}$ & Decreased thickness of load limiter plates to 0.025 in. \\
\hline 1438 & K06F & $\begin{array}{l}\text { Head mass wires start to pull away from C-spine. Left } \\
\text { femur U-bolt loose. }\end{array}$ & $\begin{array}{l}\text { Prior to test } 1438 \text {, the subject was so small that the pelvis block strap lay } \\
\text { more superiorly on pelvis such that it interacted with the pelvis } \\
\text { instrumentation/VICON mount. Strap replaced with L-bracket. }\end{array}$ \\
\hline 1439 & K06F & $\begin{array}{l}\text { Load limiter tore through entire plate engaging tether } \\
\text { cable. Head mass pulled away from C-spine. }\end{array}$ & $\begin{array}{l}\text { Increased thickness of load limiter plates to } 0.040 \text { in. Since the next } \\
\text { subject K05M had an intact head, only masking tape pre-cut prior to the } \\
\text { test was used to assist in positioning the subject. The head support bolt } \\
\text { was not used since a surrogate head mass was not used. }\end{array}$ \\
\hline 1440 & K05M & $\begin{array}{l}\text { Only tape supporting head tore, indicating minimal torso } \\
\text { movement. Left femur U-bolt loose. }\end{array}$ & \\
\hline 1441 & K05M & $\begin{array}{l}\text { One load limiter plate did not tear along scored line. } \\
\text { Caudal vertebrae rested on top of pelvis block L-bracket } \\
\text { post-test. }\end{array}$ & $\begin{array}{l}\text { Decide to run another high speed test on K02F (previously tested with no } \\
\text { fractures) with a load limiter with an ideal thickness ( } 0.040 \text { in.) for test } \\
\text { conditions. }\end{array}$ \\
\hline 1446 & $\mathrm{~K} 02 \mathrm{~F}$ & $\begin{array}{l}\text { Caudal vertebrae rested on top of pelvis block L-bracket } \\
\text { post-test. }\end{array}$ & \\
\hline
\end{tabular}


Initially the head mass of an average human 6-year-old child (3.64 $\mathrm{kg}$ ) was replicated by adding steel plates to the bolt of the head mass base. The high speed sled test (test 1435) of the trial subject (KO1F) indicated that this mass was too large for the surrogate head attachment design, as the wires broke and the head mass detached upon impact. The surrogate head base of subsequent tests were secured with more wires and the head mass was decreased for subsequent tests, using only the top head mass plate $(0.645 \mathrm{~kg})$ and surrogate head mass base (approximately $0.236 \mathrm{~kg}$ ). This resulted in a surrogate head closer to the mass of the kangaroo head (approx. $0.534 \mathrm{~kg}$ ). This mass was used for all subsequent tests.

The primary goal with the subject positioning protocol was to generate a belttorso interaction similar to that experienced by a human, including engagement of the clavicle, forward torso pitch, and belt loading through the sternum. Figure 5.5 shows the shoulder belt sliding off of the shoulder of the first trial subject test, which is one main obstacle that led to necessary test modifications.
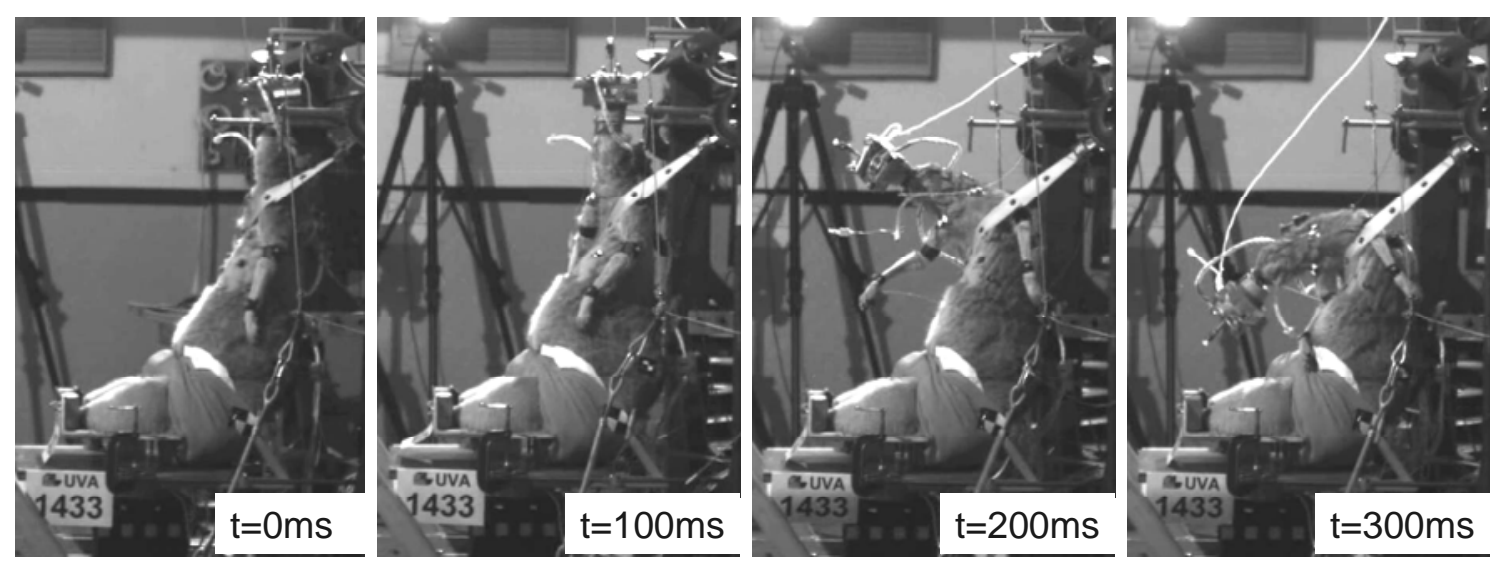

Figure 5.5: Screen shots of trial subject K01F test 1433 showing torso rotation and belt slipping off of shoulder

The narrow shoulders of the kangaroo required an upper belt anchor positioned closer to the subject than would be necessary for the broader human chest. The lateral 
position of the belt anchor site was moved towards the subject's midline $51 \mathrm{~mm}$ following test number 1433 (Figure 5.6). This horizontal belt shift was part of a series of modifications made to reduce torso twist and maintain the belt on the clavicle throughout the test.
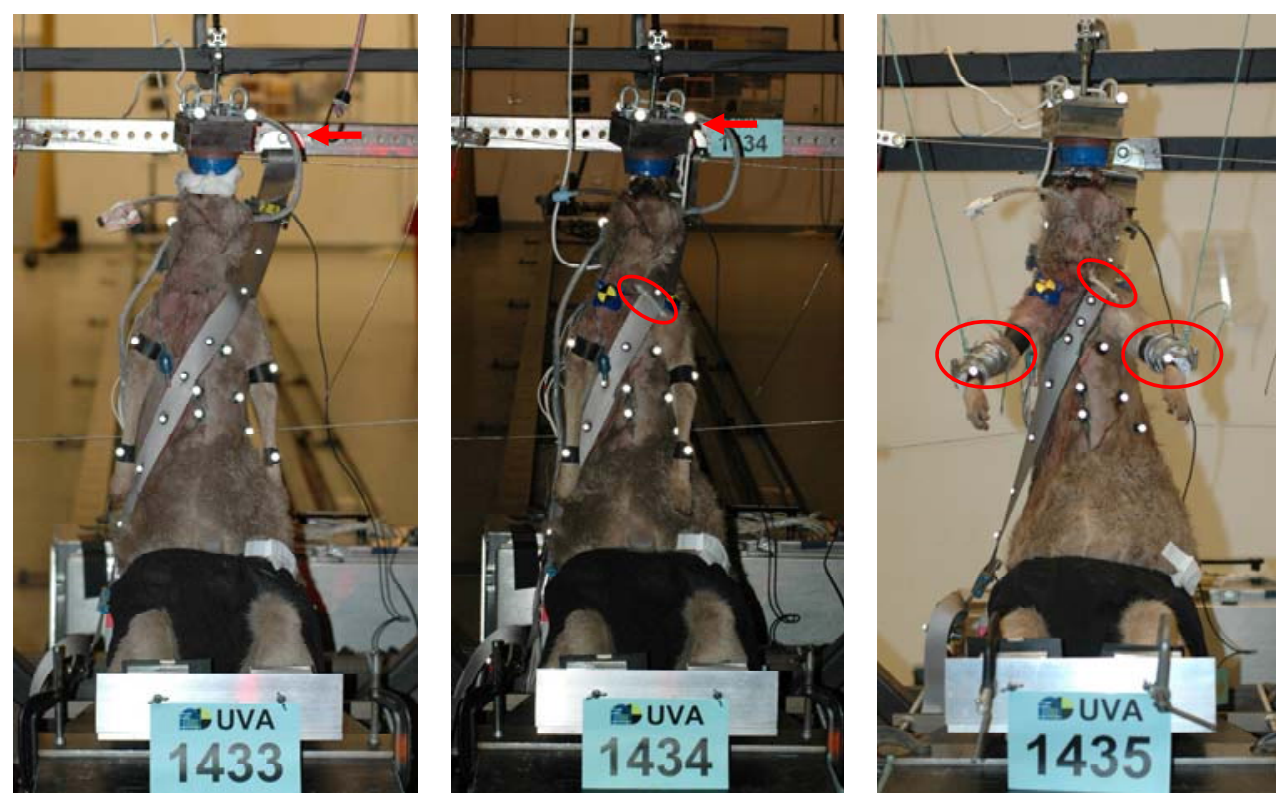

Figure 5.6: Alterations in positioning of trial subject K01F

The shoulder belt crossed the left shoulder and was constructed from restraint webbing manufactured by Narricut (details in Table 5.2). Conscious of the kangaroo's narrow shoulder breadth and short clavicles, it was determined that the standard restraint webbing would be too wide to rest on the kangaroo shoulder properly. Notching the belt (Figure 5.7) was done as part of the strategy to keep the belt engaged with the clavicle, in that it allowed the webbing to lie more medially relative to the clavicle. Belt samples with notches cut to create a dog bone shape were melted to prevent fraying and tested in a Tinius-Olson (TO) material testing machine quasistatically. The narrowed belt began to fray at loads around $8 \mathrm{kN}$, while an intact belt 
reached loads of $26 \mathrm{kN}$ before the ends pulled out of the clamping drums. The narrow portion of the belt was positioned where it crossed the clavicle, such as not to load other bony structures. Belt load cells were positioned $12 \mathrm{~cm}$ from the D-ring of upper and lower shoulder belt anchors.

After test 1433 , in addition to moving the belt anchor closer towards the subject's midline, a cable tie looped around the belt was sutured superficially to the left clavicle to help retain the position of the belt. The sutures broke during the second low speed test (test 1434) and the belt slipped off of the shoulder. To attach the cable tie loop more securely, for trial subject test 1435 , one cable tie was routed through incisions in the skin superficial to the left clavicle.

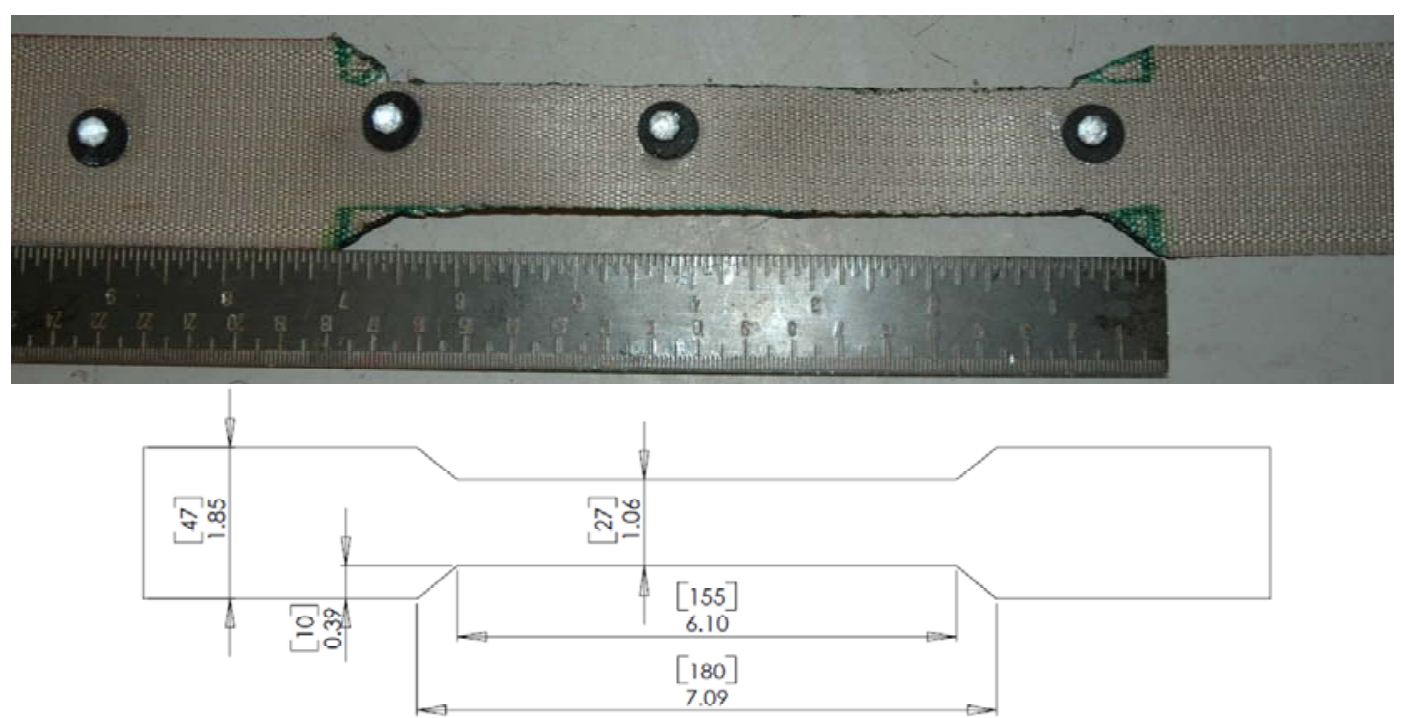

Figure 5.7: Geometry of notched shoulder belt

Additional alterations to the sled set up were performed prior to the high speed test on the trial subject (test 1435) to prevent the shoulder belt from slipping off of the shoulder. A $0.9 \mathrm{~kg}$ mass was secured to the distal aspect of each of the upper extremities and suspended below shoulder level in the transverse plane increasing the 
rotational moment of inertia, with the idea that this would help to prevent the belt from slipping off of the shoulder (Figure 5.6). The added mass consisted of a solder bar without flux $(0.45 \mathrm{~kg}, 30 \%$ Tin, $70 \%$ lead) cabled-tied to each upper extremity with lead wire $(4.88 \mathrm{~m}=0.45 \mathrm{~kg})$ looped around the bar mass. The wire was secured on both ends with hose clamps. The high speed test on the trial subject (test 1435) demonstrated that the belt alterations and arm configuration, increased the rotational moment of inertia, and succeeded in retaining the belt on the shoulder. However, the upper extremities had potential to obscure the visibility of some of the thoracic Vicon markers upon impact so the upper extremities were suspended higher and more laterally for the remaining tests following test 1435 (Figure 5.4).

The first high-speed test (1435) generated extreme lordosis throughout the thoracic and lumbar spine, and the degree of torso pitch attained by the subject in that test was less than desired. In an attempt to reduce the lordotic curvature and increase the forward torso pitch, a load-limiting element at the shoulder belt mount was employed in subsequent tests. The minimum limit for manufactured load limiters available was approximately $2.5 \mathrm{kN}$, but a force limit closer to $1.5 \mathrm{kN}$ was desired, seeing the maximum belt force on test 1435 was $2086 \mathrm{~N}$. Previous experiments at the Center for Applied Biomechanics have used a load limiter composed of two sheets of aluminum (alloy 3003) cut and scored to provide a constant force output when torn (e.g. Forman et al. 2005). An example belt load limiter used in these sled tests is shown in Figure 5.8. A tether was attached to the load limiter in case the plates tore all the way through the score lines. Various sheet thicknesses were tested quasi-statically on a Tinius-Olson (TO) 
material testing machine to determine approximate loads limits. Unfortunately, the differences in the calculated quasi-static tests behaved differently than expected under dynamic conditions, so the thickness of the load limiter sheets were adjusted throughout the kangaroo subject tests.
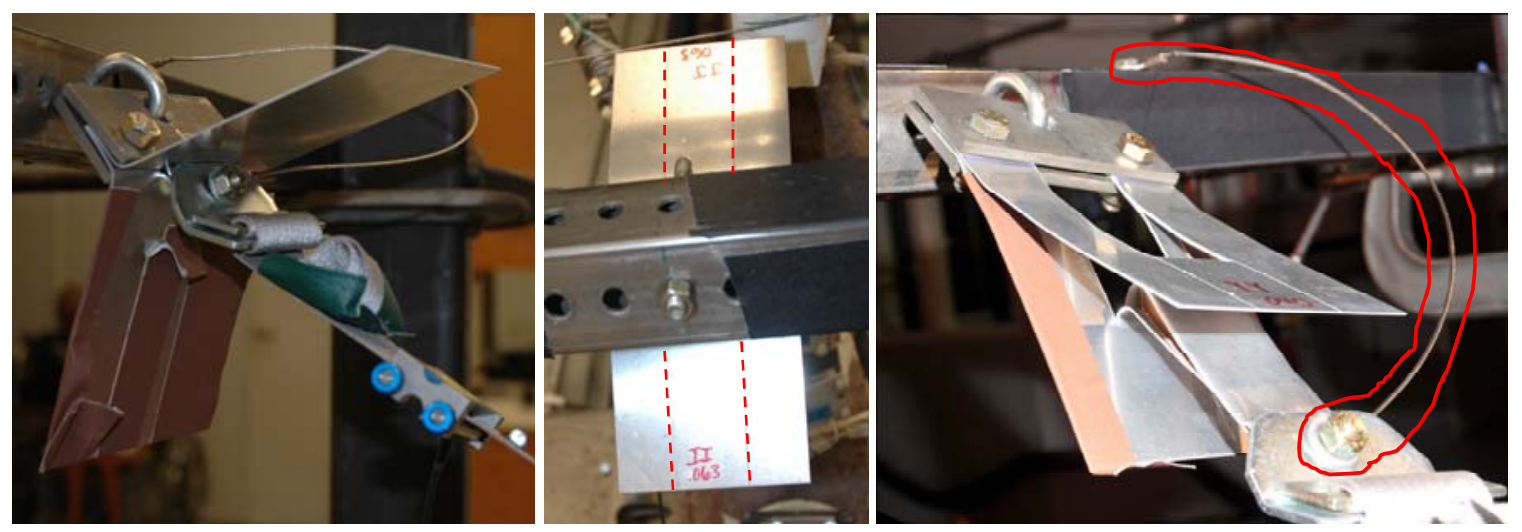

Figure 5.8: Shoulder belt load limiter with indicated score lines and safety tether circled in red.

To maintain posterior lower body positioning, a pelvis block consisting of a ratchet strap spanned two posts rigidly attached to the seat. This restricted the subject from moving posteriorly prior to impact, such that the caudal vertebrae remained on the seat surface. When testing the smallest subject, K06F, the pelvis block strap lay more superiorly on the subject, interfering with the pelvis instrumentation mount. For test 1438 , the pelvis block strap was replaced with an L-bracket secured to the back edge of the seat. This alteration was used for the remainder of the tests and provided the same restraint objective as the original strap (Figure 5.9). 

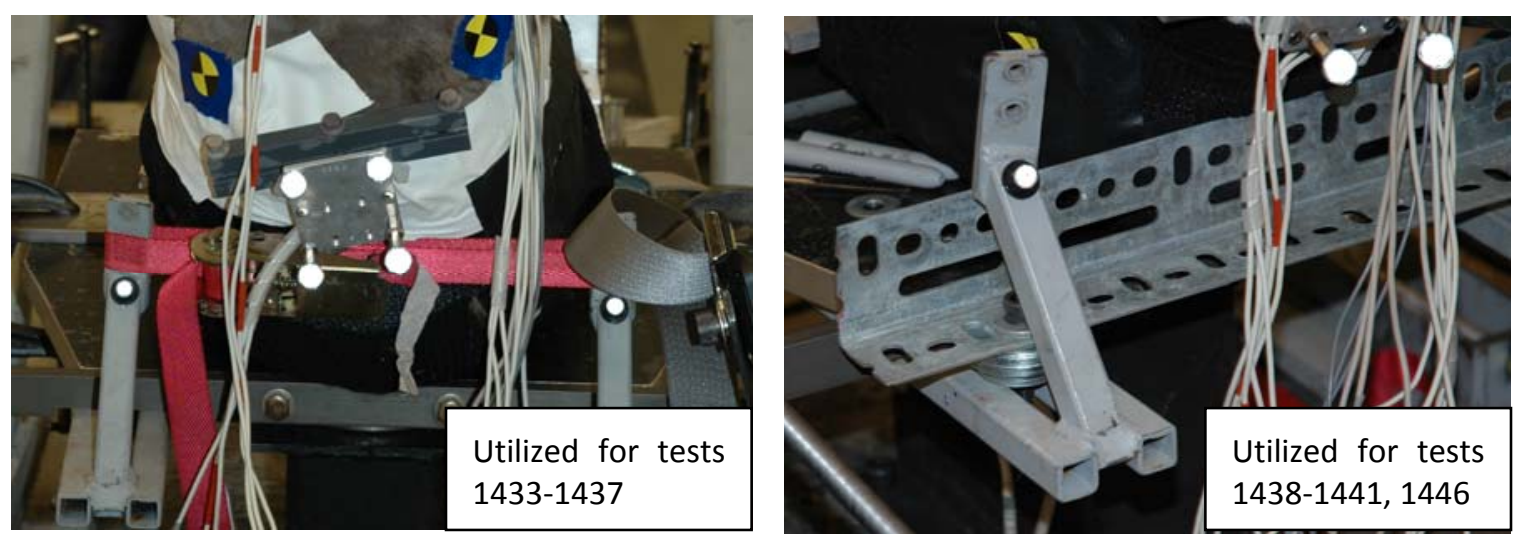

Figure 5.9: Transition from pelvis block strap to pelvis block L-bracket

\subsection{1: $\quad$ Data Analysis}

Thorax and spinal kinematics were measured using the same methodology of the Vicon motion capture system used in the hub impact tests (Section 4.2.4:). Load cells (Table 5.2) recorded the subject seat and belt loads. Tri-axial accelerometer cubes were installed on spinal mounts. Additionally, the tests were recorded using three high-speed (1000 frames/s) digital video imagers positioned for the impact view.

Instrument data was collected with TDAS, an onboard data acquisition system (Diversified Technical Systems Inc.) that acquired electronic data at $10 \mathrm{kHz}$. It was hardware-filtered to $3000 \mathrm{~Hz}$, debiased, and filtered to SAE J211-prescribed filter classes. Chest deflection was determined by the displacement between the belt marker centered on the sternum and a local coordinate system centered on the vertebral body with a Vicon array that is the most directly across from that point on the axial plane.

\section{3: Results}

Upper shoulder belt forces for low and high speed tests are shown in Figure 5.10. Two of the high speed tests were run with the same load limit. Trying different plate 
thicknesses during the first two high speed tests $(1437,1439)$ helped determine the ideal belt load limiter used in subsequent tests $(1441,1446)$.
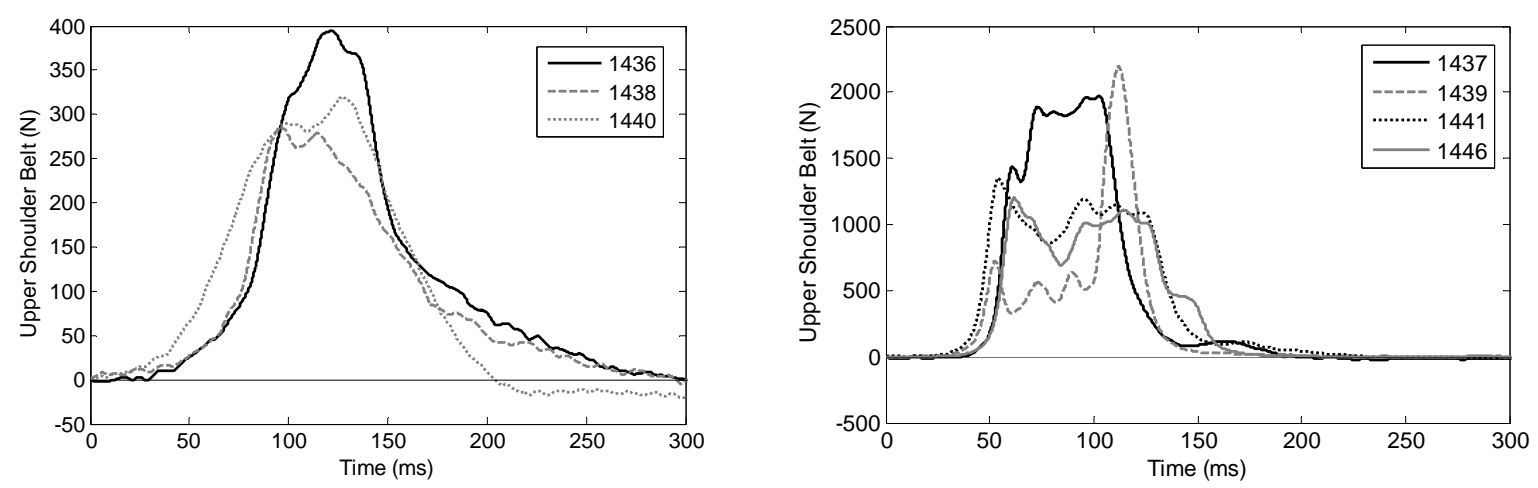

Figure 5.10: Upper shoulder belt force time history for low speed (left) and high speed (right) sled tests

The shoulder belt crossed the sternum superiorly at the manubrium and exited the thorax near the axilla region instead of more inferior similar to a human. Additionally, the vertically-positioned belt covers very little area of the anterior thorax compared to a shoulder belt on a human child. (Figure 5.11). The chest deflection data at the level of the manubrium is provided in Appendix $\mathrm{E}$.
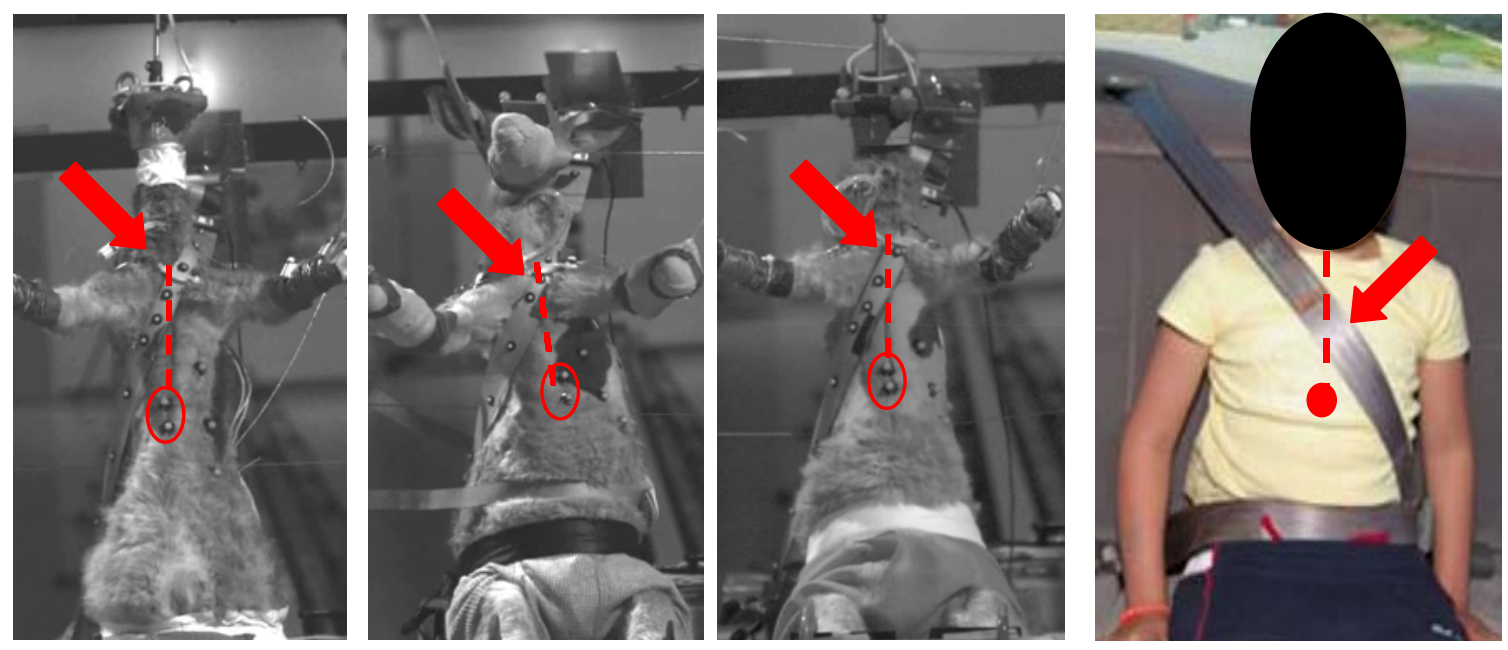

Figure 5.11: Shoulder belt positioned on K02F, K05M, K06F, and human child (from left to right) Sternum outlined with location of xiphoid process indicated by red circle and location of belt crossing sternum indicated by red arrows. 
Pronounced thoracic and lumbar lordosis was observed in each high speed test as seen in Figure 5.12. Lordosis was seen in all high speed tests regardless of whether a belt load limiter was used or the thickness of the load limiter sheets. The average peak moment in the seat about the $y$-axis was $600 \mathrm{Nm}$.

No ribcage fractures were identified in any subject during necropsy. The left clavicle of subject K06F had a displaced fracture most likely due to the shoulder belt tearing through the load limiter and engaging the tether cable at a sharp peak force of $2193 \mathrm{~N}$ in high speed test 1439 (Figure 5.10). Spinal and internal organ injuries were also assessed, but no injuries were found.
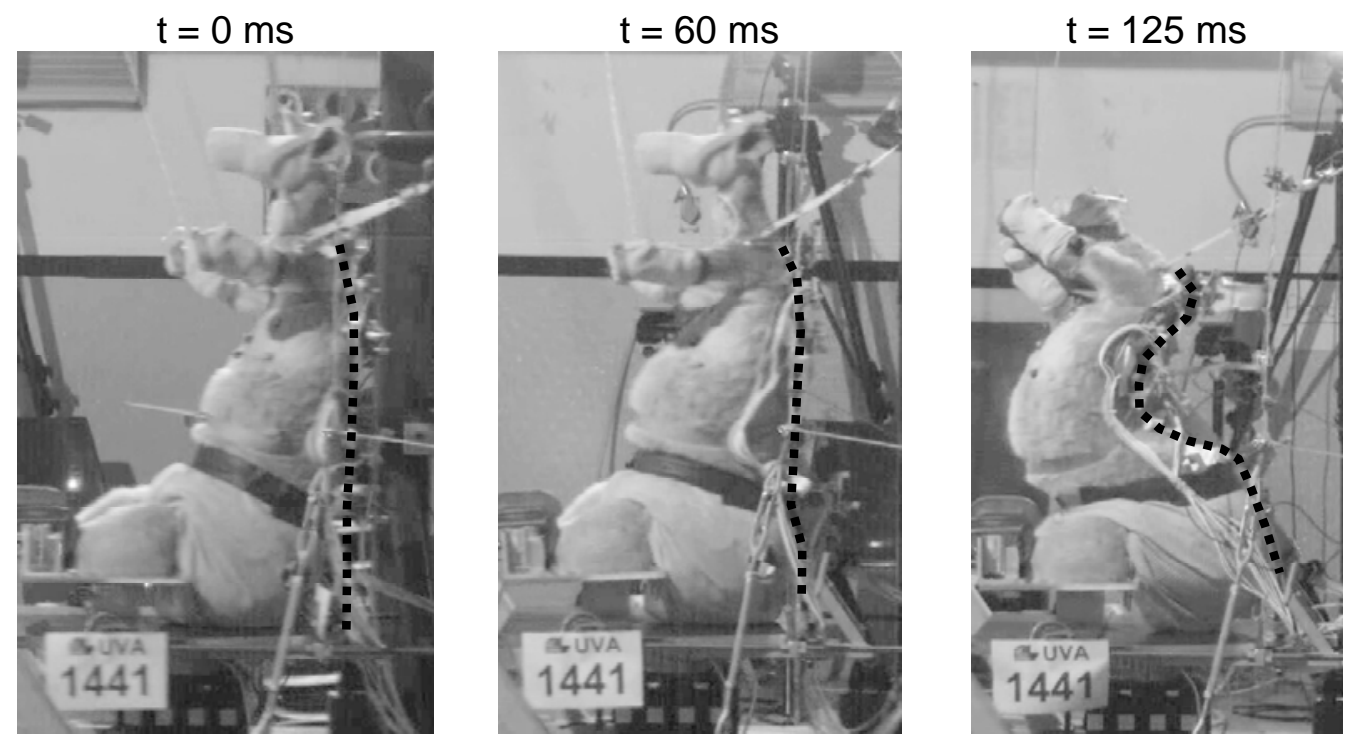

Figure 5.12: Motion of K05M during high speed test

\section{4: Discussion}

The low speed shoulder belt forces seen in the kangaroo are comparable to those seen in 6-8 year-old human volunteers exposed to the same deceleration pulse (Arbogast et al. 2009). There is no comparable low speed volunteer chest deflection 
data to date. The usefulness of the chest deflection response from the kangaroo is not clear. The deflection measuring the position of the manubrium (where the belt crossed the sternum) with respect to the spine is not analogous to the chest deflection instrumentation on ATDs (positioned mid-sternum). The deflection of a superiorly positioned belt also would not be representative of a belt positioned mid-sternum on a child. A superiorly positioned belt engages stiffer boney structures including the short first ribs as well as the clavicles.

This study shows that the kangaroo is limited as a model of a pediatric human in a high speed sled test because of differences in thoracic mass distribution, the length of the spine, and the belt positioning. The kangaroo has narrower shoulders and a broader pelvic region compared to human children. Kangaroos have an additional thoracic (T13) and lumbar vertebrae (L6) producing more degrees of freedom in the spine and all of their vertebral bodies are longer compared to those of humans resulting in a longer torso length. The unique geometry of the kangaroo torso limited the shoulder belt to lie superiorly on the thorax (i.e., further from the effective torso center of mass) likely contributing to marked spinal lordosis. It is probable that the degree of lordosis observed is larger than a human would exhibit. Lordosis can, however, occur in humans. Shaw et al. (2001) showed thoracic lordosis in an unbelted subject loaded superiorly on the thorax by an airbag, and pediatric PMHS from Kallieris et al (1976) exhibited some lordosis when a shoulder belt was positioned high on the thorax. 


\section{5: Summary}

This study evaluated the response of four juvenile kangaroo carcasses in low speed $(9 \mathrm{~km} / \mathrm{h})$ and high speed $(39 \mathrm{~km} / \mathrm{h})$ frontal sled tests. The subjects were restrained on a rigid planar seat by a custom designed pelvis restraint and a shoulder belt with a load limiter. Restraint geometry differed from standard vehicle restraints to accommodate the unique torso geometry of the kangaroo.

The unique geometry of the kangaroo torso required several alterations in the test set-up, which were made in order to create a belt-torso interaction as human-like as possible (decreasing head mass, increasing torso polar moment-of-inertia, notching shoulder belt, routing shoulder belt, moving belt D-ring medially, and adding a belt load limiter). Despite these efforts, substantial spinal lordosis was observed during the high speed impacts, which is unlikely to represent the lordosis that would be generated in a human exposed to this impact environment, at least in degree. Presumably this lordosis results partially from the relatively long and conical-shaped torso of the kangaroo. These sled tests show that the kangaroo is not a good model of the pediatric thorax under a belt restraint in an inertially accelerated environment. These sled tests confirmed the importance of geometric similitude, in particular torso geometry and mass distribution when testing in an inertial loading conditions. 


\section{Chapter 6: Conclusions}

\section{1: Summary}

Due to the lack of pediatric thoracic biomechanical response data in high-speed frontal crashes and the difficulty in obtaining pediatric PMHS, this thesis assessed the eastern grey kangaroo as a biomechanical model of a human six-year-old under frontal loading. This assessment consisted of four specific aims. First, juvenile kangaroo carcasses were obtained and compared to humans through a detailed anatomical study. Then the kangaroos were subjected to three frontal loading conditions. Two of the experiments were non-injurious cyclic CPR loading and blunt frontal impacts to replicate existing pediatric thoracic biomechanical data for comparison. Finally, the kangaroos were tested in the loading environment of interest; a belt restraint in an accelerated environment.

The kangaroo thoracic response was comparable to the pediatric PMHS and volunteer data in the CPR loading environment. The CPR loading geometry was not representative of a belt restraint and the loading rate was an order of magnitude lower than that observed in crashes. The higher loading rate blunt hub impact tests produced thoracic force-deflection curves for the kangaroos that did not show a large inertial force at impact compared to the pediatric PMHS, such that the work done by the kangaroo deformation was approximately a third less than the pediatric subjects. Challenges of the kangaroo sled tests included ill-fitting belt positioning and extreme lumbar lordosis due to the narrow shoulder breadth, long torso length, and lower center of gravity. At the conclusion of the sled tests, it was determined that the kangaroo is 
not a good model of the pediatric thorax for belt loading in frontal impact. Below is a summary of strengths and limitations of the kangaroo as a model of the pediatric thorax.

\section{2: Strengths of the Juvenile Kangaroo as a Biomechanical Model of the Human 6-} Year-Old's Thorax

The eastern grey kangaroo met many general characteristics considered important for a biomechanical model of the child for frontal belt loading prior to experimental tests. Desired characteristics for the biomechanical model included availability and geometric, length, mass, and modulus similitude. Availability was not an issue due to the abundance of kangaroos that led to culls in Australia. The similitude traits were needed based on the uncertainty of scaling methods that have been used to develop pediatric ATDS and computational models.

The kangaroo possesses many anatomical attributes for geometric similitude. As one of a few species that occasionally walk or hop bipedally, the semi-erect posture contributes to the spinal curvature and organ positioning. Also, the kangaroo's arrangement of costal cartilage from the ribs to sternum is the same as human, but the kangaroos have an additional floating rib. Lastly, kangaroos have clavicles, which are an important load path for shoulder belts.

To minimize the need for modulus scaling the kangaroo was determined to be around the size (by mass) of a six-year-old human when at the equivalent developmental stage of a six-year-old human. 


\section{3: Limitations of the Juvenile Kangaroo as a Biomechanical Model of the Human 6-year-old Thorax}

Although there are some similar anatomical features between the eastern grey kangaroo and humans, the overall torso geometry and distribution of thoracic mass was not representative of humans for the experimental thoracic loading conditions.

The conical shape of the kangaroo torso affected the CPR tests and the blunt hub impact tests. The difference between the thoracic circumference at the axilla region and the pelvic region was much greater for kangaroos than humans. The conical kangaroo torso placed supine on a flat posterior boundary condition led to steep sternum angles for the placement of the CPR FDS puck. The effect of the force vector orthogonal to spine, but not orthogonal to the sternum was uncertain. In the hub impact tests, the conical shape presented challenges in replicating the positioning criteria from Ouyang et al. (2006). If the hub surface was parallel to the sternum then the spine was not vertical.

Related to the conical shape of the kangaroo torso is the narrow shoulder breadth and narrow upper thorax of the kangaroos. While kangaroos do possess clavicles, they are very short clavicles. The first five pairs of ribs lateral to the length of the sternum are relatively short with thin curved costal cartilage to connect the ribs to the sternum. The geometry of the CPR FDS puck and the blunt hub surface loaded the majority of the anterior thorax surface area of the kangaroos compared to those same loading geometries on the pediatric thorax. The narrow shoulder breadth and upper thorax region of the kangaroo led to difficulties in belt positioning for the sled tests. The 
clavicle was narrower than typical seat belt webbing so the belt was narrowed to not load other bony structures. The narrow shoulder breadth also caused the kangaroo to rotate out of the shoulder belt easily. An animal with a narrow shoulder breadth is not ideal for shoulder belt loading due to its smaller rotational moment of inertia compared to a child with broader shoulders.

The lengths of the juvenile kangaroo torsos obtained were significantly longer than an average human six-year-old. Although the mass of the subjects were comparable to human six-year-olds, the center of gravity of the kangaroos was situated more caudally. The affects of torso length combined with the distribution of mass of the kangaroo on spinal kinematics was first apparent in the hub impact tests. Post impact, the kangaroos in the hub impacts tipped over rotating on the pelvis. The long torso length contributed to the ill-fitting shoulder belt such that it restrained only the most superior portion of the thorax and was oriented more vertically. The torso length accentuated by the lower center of gravity of the kangaroo led to pronounced lordosis in the high speed sled tests. The torso length and mass distribution play a significant role in subject kinematics, and they were also a main limiting factor for the kangaroo's use as a biomechanical model of the pediatric thorax.

\section{4: Contributions and Future Work}

Assessing the kangaroo as a model of the pediatric thorax was a novel idea and this is the first evaluation of the kangaroo as a biomechanical model of the pediatric thorax. Unfortunately, the experimental tests did not support the hypothesis for all the thoracic loading conditions. The kangaroo had a human-like thoracic response to CPR 
loading; however, there were significant differences in thoracic response in blunt hub impacts between the kangaroos and pediatric PMHS. Additionally, the kangaroo belt positioning and kinematic response in high-speed frontal impacts did not seem reasonable for a human child and therefore the kangaroo is not a viable model of the pediatric thorax for belt restraint frontal loading.

Throughout the assessment of the kangaroo, this thesis justifies the importance of geometric similitude and mass distribution and their effects on structural behavior for belt loads in an inertial environment. It was not possible to don a belt on a kangaroo in a human-like manner due to the torso geometry. When the belt positioning was forced on the kangaroo, it resulted in un-human-like spinal kinematics.

The strengths of the kangaroo are reasonable characteristics to consider when finding surrogates for other biomechanical tests, i.e. growth development to minimize modulus scaling and anatomical features necessary for loading of interest. In the case of the kangaroos, differences in some aspects of geometric similitude trumped the advantages of anatomical similarities and a similar age and growth development.

Seeing that the kangaroo is not a good model for belt loading in a sled test, there does not appear to be another available animal model that would fulfill important criteria for belted frontal loading, while minimizing the need for scaling. The role of animals in the continuation of pediatric biomechanics research is uncertain. The conclusion of this study leaves the state of the field of pediatric biomechanics with a limited amount of pediatric data. However, this study does highlight important characteristics for the continuing development of pediatric biofidelic tools, i.e. ATDs and 
computational models. The current 6-year old ATDs have differences in thoracic mass and geometry and anatomical features are not very refined. Although there are inconsistencies between the current ATDs, their continued development through computational models can take into account the importance of geometric and mass similitude. The restraint interaction is important for thoracic structural response, where the geometric similitude of the torso plays a key role in proper restraint interaction. Development of the models with consideration of mass similitude is also important so the distribution of mass and center of gravity produce a biofidelic response in an inertial environment. 


\section{References}

Aldman, B., Andersson, A., \& Saxmark, O. (1974). Possible effects of airbag inflation on a standing child., (pp. 194-215).

Alexander, R. M. (2004). Bipedal animals, and their differences from humans. J. Anatomy, 204 (5), 321-330.

Arbogast, K. B., Balasubramanian, S., Seacrist, T., Maltese, M. R., Garcia-Espana, J. F., Hopely, T., et al. (2009). Comparison of kinematic responses of the head and spine for children and adults in low-speed frontal sled tests. Stapp Car Crash J , 53, 329372.

Arbogast, K. B., Cornejo, R. A., Kallan, M. J., Winston, F. K., \& Durbin, D. R. (2002). Injuries to children in forward facing child restraints. Annu Proc Assoc Adv Automot Med, 46, 213-230.

Arbogast, K. B., Durbin, D. R., Kallan, M. J., \& Winston, F. K. (2004). Evaluation of pediatric use patterns and performance of lap shoulder belt systems in the center rear. Annu Proc Assoc Adv Automot Med , 48, 57-72.

Asdell, S. A. (1964). Patterns of mammalian reproduction. Comstock Publishing Associates.

Backaitis, S., Medlin, J., Radovich, V., Stalnaker, R., Shah, M., Shaffer, J., et al. (1975). Performance evaluation of child dummies and baboons in child restraint systems in a systemized crash environment. Society of Automotive Engineers, 751153, 345-403.

Beckman, D. L., \& Palmer, M. F. (1970). Thoracic force-deflection studies in primates. J Biomech , 3 (2), 223-227.

Brun-Cassan, F., Page, M., Pincemaille, Y., Kalliris, D., \& Tarriere, C. (1993). Comparative Study of Restrained Child Dummies and Cadavers in Experimental Crashes. Society of Automotive Engineers, 933105, 243-260.

Burdi, A., Huelke, D., Snyder, R., \& Lowery, G. (1969). Infants and children in the adult world of automobile safety design: pediatric and anatomical considerations for design of child restraints. J Biomech , 2 (3), 267-280.

Cappozzo, A., A, C., Croce, U., \& Pensalfini, F. (1997). Surface-marker Cluster Design Criteria for 3D Bone Movement Reconstruction. IEEE Transactions on Biomedical Engineering , 44 (12), 1165-1174.

CDC. (2011). Child Passenger Safety: Fact Sheet. Child Passenger Safety: Fact Sheet .

CDC. (2007). Ten leading causes of Injury Deaths by Age Group Highlighing Unintentional Injury Deaths, United States-2007. Ten leading causes of Injury Deaths by Age Group Highlighing Unintentional Injury Deaths, United States-2007 . 
Codman, E. (1934). The Shoulder: rupture of the supraspinatus tendon and other lesions in or about the subacromial bursa. T. Todd company.

Coelho, A., Glassman, D., \& Bramblett, C. (1984). The relation of adiposity and body size to chronological age in olive baboons. Growth , 48 (4), 445-454.

Crandall, J. R., Bose, D., Forman, J., Untaroiu, C. D., Arregui-Dalmases, C., Shaw, C. G., et al. (2011). Human surrogates for injury biomechanics research. Clin Anat, 24 (3), 362-371.

Dawson, T. (1995). Kangaroos: Biology of the Largest Marsupials. Cornell University Press.

Dejeammes, M., Tarriere, C., Thomas, C., \& Kallieris, D. (1984). Exploration of Biomechanical Data Towards a Better Evaluation of Telerance for Children Involved in Automotive Accidents. Society of Automotive Engineers , 840530, 427-440.

DeLamater, J., \& Friedrich, W. (2002). Human Sexual Development. Journal of Sex Research , 39 (1), 10-14.

Eppinger, R., Sun, E., Kuppa, S., \& Saul, R. (2000). Supplement: Development of Improved Injury Criteria for the Assessment of Advanced Automotive Restraint Systems - II. 43p - .

Forbes, P. (2008). Development of child human body models and simulated testing environments for the imporvement of child safety. TNO-033-HM-2007-00311.

Forman, J., Kent, R., Ali, T., Crandall, J., Bostrom, O., \& Haland, Y. (2005). Biomechanical Considerations for the Optimization of an Advanced Restraint System: Assessing the Benefit of a Second Shoulder Belt.

Forman, J., Lessley, D., Kent, R., Bostrom, O., \& Pipkorn, B. (2006). Whole-body kinematic and dynamic response of restrained PMHS in frontal sled tests. Stapp Car Crash J , 50, 299-336.

Forman, J., Lessley, D., Shaw, C. G., Evans, J., Kent, R., Rouhana, S. W., et al. (2006). Thoracic response of belted PMHS, the Hybrid III, and the THOR-NT mid-sized male surrogates in low speed, frontal crashes. Stapp Car Crash J , 50, 191-215.

Franklyn, M., Peiris, S., Huber, C., \& Yang, K. H. (2007). Pediatric material properties: a review of human child and animal surrogates. Crit Rev Biomed Eng , 35 (3-4), 197342.

Frith, H., \& Calaby, J. (1969). Kangaroos. (H. Press, Ed.) Hurst and Company.

Gavan, J. (1953). Growth and Development of the chimpanzee; a longitudinal and comparative study. Human biology, 25, 93-143.

Glassman, D., Coelho, A., Carey, K., \& Bramblett, C. (1984). Weight growth in savannah baboons: a longitudinal study from birth to adulthood. Growth , 48, 425-433.

Hopwood, P. (1976). The Quantitative Anatomy of the Kangaroo. University of Sydney. 
Inan, M., Ayvaz, S., Sut, N., Aksu, B., \& U, T. B. (2007). Blunt Chest Trauma in Childhood. ANZ J. Surg, 77, 682-685.

Irwin, A., \& Mertz, H. (1997). Biomechanical basis for the CRABI and Hybrid III child dummies. In S. o. Automotive (Ed.)., (pp. 261-272).

IUCN2012. (n.d.). IUCN Red List of Threatened Species. Version 2012.2. IUCN Red List of Threatened Species. Version 2012.2 .

Kallieris, D., Barz, J., Schimdt, G., \& Heess, G. (1976). Comparison between Child Cadavers and child dummy by using child restraint systems in simulated collisions. SAE International (760815), 513-542.

Kallieris, D., Schimdt, G., Barz, J., Mattern, R., \& F, S. (1978). Response and Vulnerability of the Human Body at Different Impact Velocities in Simulated Three-point Belted Cadaver Tests.

Kent, R., Lessley, D., \& Sherwood, C. (2004). Thoracic response to dynamic, non-impact loading from a hub, distributed belt, diagonal belt, and double diagonal belts. Stapp Car Crash J , 48, 495-519.

Kent, R., Lopez-Valdes, F., Lamp, J., Lau, S., Parent, D., Kerrigan, J., et al. (2012). Biomechanical response targets for physical and computational models of the pediatric trunk. Traffic Inj Prev .

Kent, R., Lopez-Valdes, F., Lau, S., Kerrigan, J., \& Lessley, D. (2011). Thoracic Response of Pediatric PMHS. SAE International , 5248.

Kent, R., Salzar, R., Kerrigan, J., Parent, D., Lessley, D., Sochor, M., et al. (2009). Pediatric thoracoabdominal biomechanics. Stapp Car Crash J , 53, 373-401.

Kent, R., Stacey, S., Kindig, M., Forman, J., Woods, W., Rouhana, S. W., et al. (2006). Biomechanical response of the pediatric abdomen, part 1: development of an experimental model and quantification of structural response to dynamic belt loading. Stapp Car Crash J , 50, 1-26.

Kent, R., Stacey, S., Kindig, M., Woods, W., Evans, J., Rouhana, S. W., et al. (2008). Biomechanical response of the pediatric abdomen, Part 2: injuries and their correlation with engineering parameters. Stapp Car Crash J , 52, 135-166.

Kirkpatrick, T. (1967). The Grey Kangaroo in Queensland. Queensland Agricultural Journal , 550-552.

Lessley, D., Salzar, R., Crandall, J., Kent, R., Bolton, J., Bass, C., et al. (2010). Kinematics of the thorax under dynamic belt loading conditions. International Journal of Crashworthiness, 15 (2), 175-90.

Lessley, D., Shaw, G., Riley, P., Forman, J., \& J., C. (2011). Assessment and Validation of a Methodology for Measuring Anatomical Kinematics of Restrained Occupants During MOtor Vehicle Collisions. J. Biosensors and Bioelectronics, S1:002. 
Life, J., \& Pince, B. (1968). Response of the canine heart to thoracic impact during ventricular diastole and systole. J. Biomechanics, 1, 169-173.

Lopez-Valdes, F. J., Forman, J., Kent, R., Bostrom, O., \& Segui-Gomez, M. (2009). A comparison between a child-size PMHS and the Hybrid III 6 YO in a sled frontal impact. Ann Adv Automot Med, 53, 237-246.

Lopez-Valdes, F. J., Lau, A., Lamp, J., Riley, P., Lessley, D. J., Damon, A., et al. (2010). Analysis of spinal motion and loads during frontal impacts. Comparison between PMHS and ATD. Ann Adv Automot Med , 54, 61-78.

Lu, Y., Chen, C., Kallakuri, S., Patwardhan, A., \& Cavanaugh, J. M. (2005). Development of an in vivo method to investigate biomechanical and neurophysiological properties of spine facet joint capsules. Eur Spine J , 14 (6), 565-572.

Luck, J., Nightingale, R., Loyd, A., Prange, M., Dibb, A., Song, Y., et al. (2008). Tensile Mechanical Properties of the Perinatal and Pediatric PMHS Osteoligamentous Cervical Spine. Stapp Car Crash journal , 52, 107-134.

Maltese, M. R., Castner, T., Niles, D., Nishisaki, A., Balasubramanian, S., Nysaether, J., et al. (2008). Methods for determining pediatric thoracic force-deflection characteristics from cardiopulmonary resuscitation. Stapp Car Crash J , 52, 83-105.

Mattern, R., Kallieris, D., Riedl, H., \& Wiren, B. v. (2002). Reanalysis of two child PMHStests. University of Heidleberg.

Mertz, H., Driscoll, G., Lenox, J., Nyquist, G., \& Weber, D. (1982). Responses of animals exposed to deployment of various inflatable restraint system concepts for a variety of collision severities and animal positions., (pp. 352-368).

Napier, J., \& Napier, P. (1985). The natural history of the primates. The MIT Press, Cambridge, Massachusetts.

NHTSA. (2009). Traffic Safety Facts 2009: Children. Techincal Report, National Highway Traffic Safety Administration.

Nowak, R. (1999). Walker's Mammals of the World. The Johns Hopkins University Press.

Ouyang, J., Zhao, W., Xu, Y., Chen, W., \& Zhong, S. (2006). Thoracic impact testing of pediatric cadaveric subjects. J Trauma , 61 (6), 1492-1500.

Parent, D. P., Crandall, J. R., Bolton, J. R., Bass, C. R., Ouyang, J., \& Lau, S. H. (2010). Comparison of Hybrid III child test dummies to pediatric PMHS in blunt thoracic impact response. Traffic Inj Prev, 11 (4), 399-410.

Parent, D. (2008). Scaling and Optimization of Thoracic Impact Response in Pediatric Subjects. Master's Thesis, University of Virgina.

Patrick, L., \& Nyquist, G. (1972). Airbag effects on the out-of-position child. Society of Automotive Engineers, 720442.

Poole, W. (1982). Macropus giganteus. Mammalian Species, 187, 1-8. 
Poole, W., \& Catling, P. C. (1974). Reproduction in the Two Species of Grey Kangaroos, Macropus giganteus Shaw and M. fuliginosus (Desmarest) I. Sexual Maturity and Oestrus. Austr. J. Zool. , 22, 277-302.

Prasad, P., \& Daniel, R. (1984). A biomechanical analysis of head, neck, and torso injuries to child surrogates due to sudden torso acceleration. Society of Automotive Engineers, 841656, 25-40.

Roberts, V., Jackson, F., \& Berkas, E. (1966). Heart Motion Due to Blunt Trauma to the Thorax.

Rogol, A., Roemmich, J., \& Clark, P. (2002). Growth at Puberty. Journal of Adolescent Health, 31, 192-200.

Russell, E. (1974). The biology of Kangaroos (Marsupialia-Macropodidae). Mammal Review , 4 (1/2), 1-59.

Schreck, R., \& Patrick, L. (1975). Frontal crash evaluation tests of a five-point harness child restraint. Society of Automotive Engineers, 751152.

Seacrist, T., Balasubramanian, S., Garc $\{\backslash$ 'i\}a-Espa $\{\backslash \sim$ n\}a, J. F., Maltese, M. R., Arbogast, K. B., Lopez-Valdes, F. J., et al. (2010). Kinematic Comparison of Pediatric Human Volunteers and the Hybrid III 6-Year-Old Anthropomorphic Test Device. Ann Adv Automot Med, 54, 97-108.

Seacrist, T., Samuels, M., Garcia-Espana, J., Arbogast, K., Mathews, E., Balasubramanian, S., et al. (2012). Kinematic Comparison of the Hybrid III and Q-Series Pediatric ATDs to Pediatric Volunteers in Low-Speed Frontal Crashes. Ann Adv Automot Med, 56, 285-298.

Shatsky, S., Alter, W., Evans, D., Armbrustmacher, V., \& Clark, G. (1974). Traumatic Distortions of the Primate Head and Chest: Correlation of Biomechanical, Radiological and Pathological Data.

Shaw, C. G., Kent, R., Sieveka, E., \& Crandall, J. (2001). Spinal kinematics of restrained occupants in frontal impacts.

Shaw, G., Parent, D., Purtsezov, S., Lessley, D., Crandall, J., Kent, R., et al. (2009). Impact response of restrained PMHS in frontal sled tests: skeletal deformation patterns under seat belt loading. Stapp Car Crash J , 53, 1-48.

Sherwood, C. P., Ferguson, S. A., \& Crandall, J. R. (2003). Factors leading to crash fatalities to children in child restraints. Annu Proc Assoc Adv Automot Med , 47, 343359.

Sherwood, C., Shaw, C., Rooij, L. V., Crandall, J., Orzechowski, K., Eichelberger, M., et al. (2003). Prediction of cervical spine injury risk for the 6-year-old child in frontal crashes. Traffic Inj Prev , 4, 206-213. 
Snyder, R., Schneider, L., Owings, C., Reynolds, H., Golomb, D., \& Schork, M. (1977). Anthropoemtry of Infants, Children and Youths to age 18 for Product Safety Design. U.S. Consumer Product Safety Commission Final Report. UM-HSRI-77-17.

Sorensen, G. N. (1906). A Comparison of the Skeleton of Man with the Skeleton of the Kangaroo. University of Utah.

Stocker, J., \& Dehner, L. P. (2001). Pediatric Pathology. (J. Stocker, \& L. P. Dehner, Eds.) Lippincott Williams and Wilkins.

Sturtz, G. (1980). Biomechanical data of children.

Tribe, D., \& Peel, L. (1963). Body Composition of the Kangaroo. Aust J Zool , 11, 273-289.

Viano, D. C., \& Warner, C. Y. (1976). Thoracic Impact Response of Live Porcine Subjects.

Wismans, J., Maltha, J., Melvin, J., \& Stalnaker, R. (1979). Child restraint evaluation by experimental and mathematical simulation. Society of Automotive Engineers, 791017, 383-415.

Zacharias, L., \& Wurtman, R. (1969). Age at menarche. New England Journal of Medicine , $280(16), 868-875$.

Zdeblick, T. A., Cooke, M. E., Wilson, D., Kunz, D. N., \& McCabe, R. (1993). Anterior cervical discectomy, fusion, and plating. A comparative animal study. Spine (Phila Pa 1976) , 18 (14), 1974-1983. 


\section{Appendix A: Flexibility of Kangaroo Rib Cage}

Images demonstrating flexibility of denuded, eviscerated K02F rib cage. The large intercostals spacing with respect to rib diameter is also noticeable.

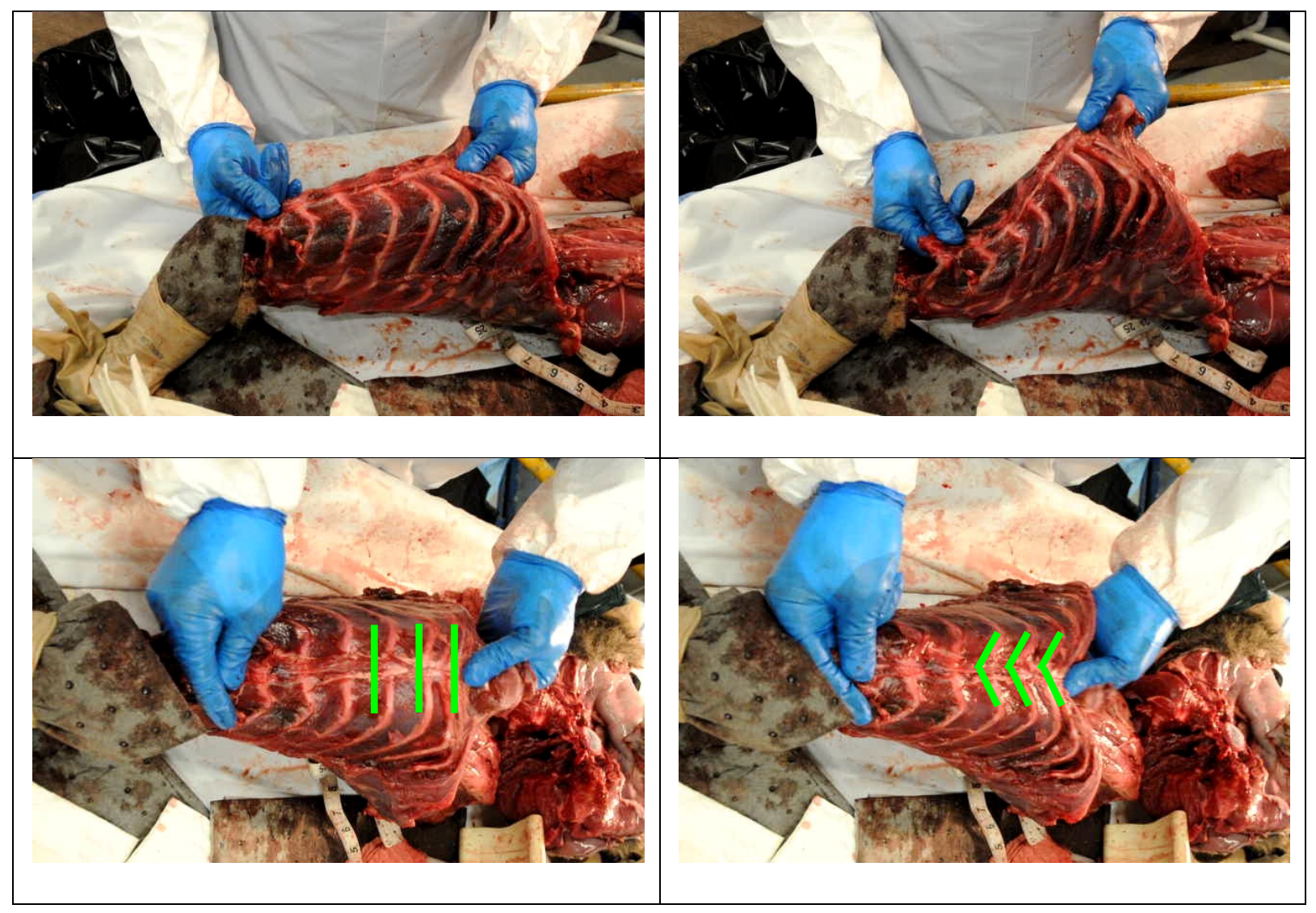




\section{Appendix B: CPR Force-deflection Cycles and Thoracic}

\section{Model Coefficients}

Figures are zeroed force-deflection cycles and the elastic force at $10 \%$ of the subjects' chest depth. The black link is the elastic force from the model stiffness coefficients.

Table B1 provides thoracic model coefficients and linear stiffness values.

\section{Subject K01F}

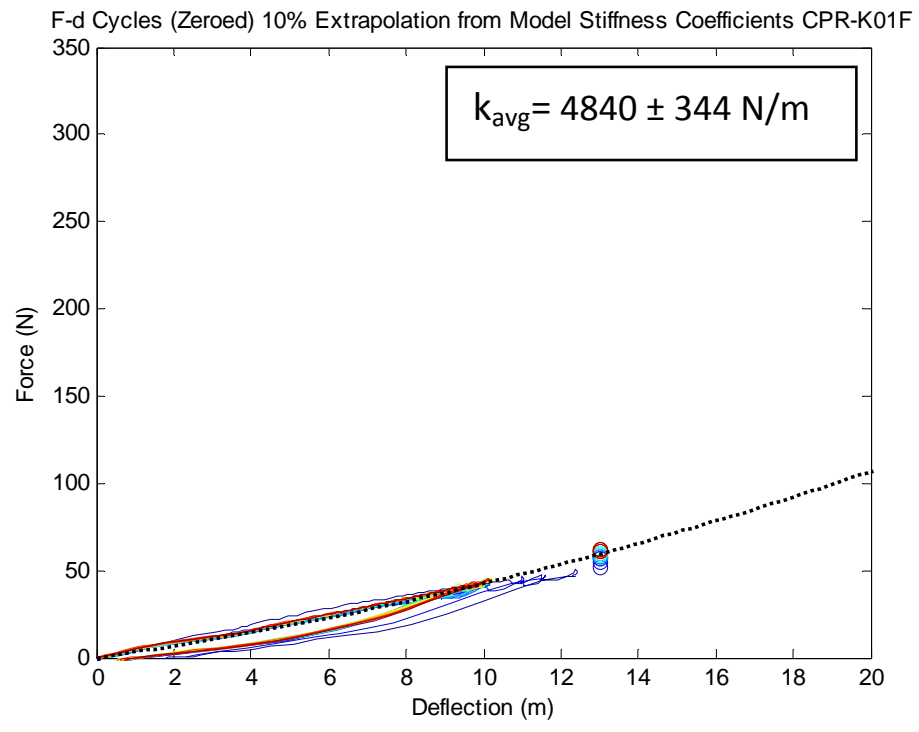




\section{Subject K02F}

F-d Cycles (Zeroed) 10\% Extrapolation from Model Stiffness Coefficients CPR-K02F-1-10
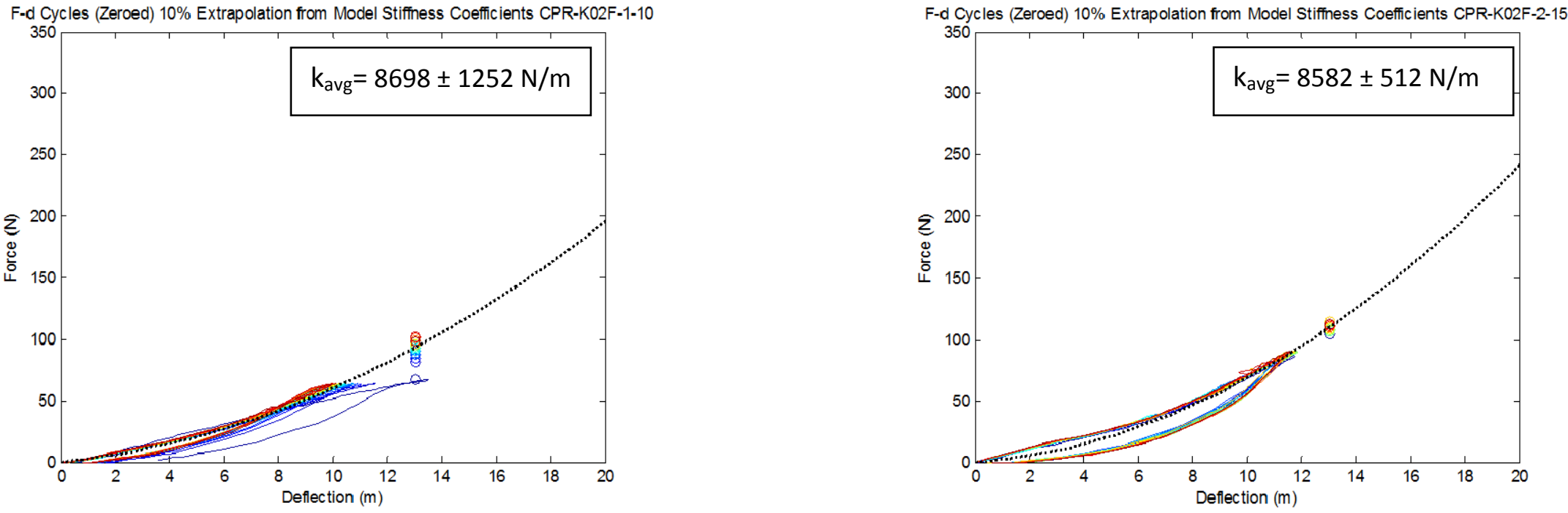

F-d Cycles (Zeroed) 10\% Extrapolation from Model Stiffness Coefficients CPR-K02F-3-10

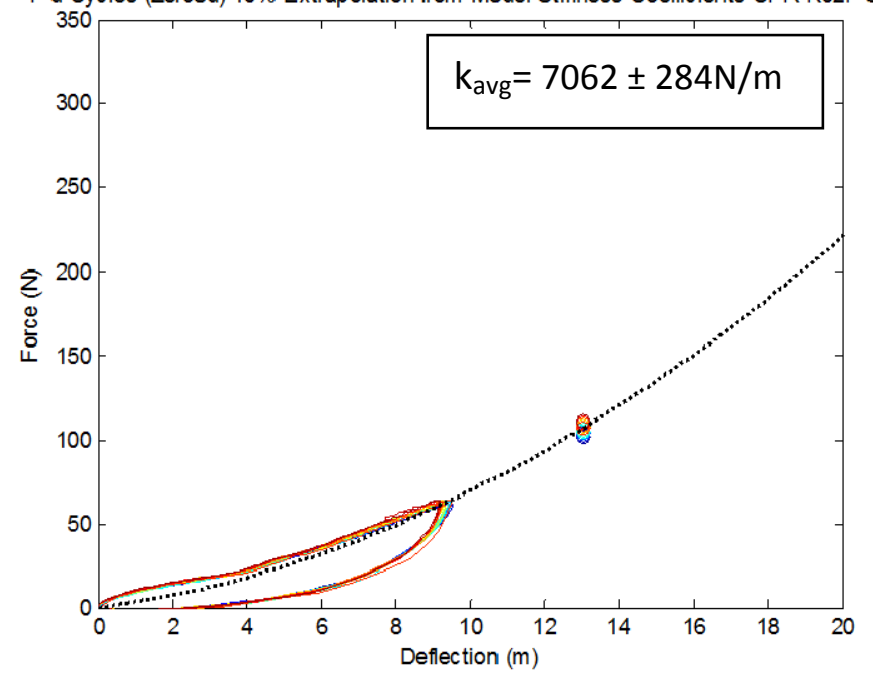




\section{Subject K05M}

F-d Cycles (Zeroed) 10\% Extrapolation from Model Stiffness Coefficients CPR-K05M-1-10
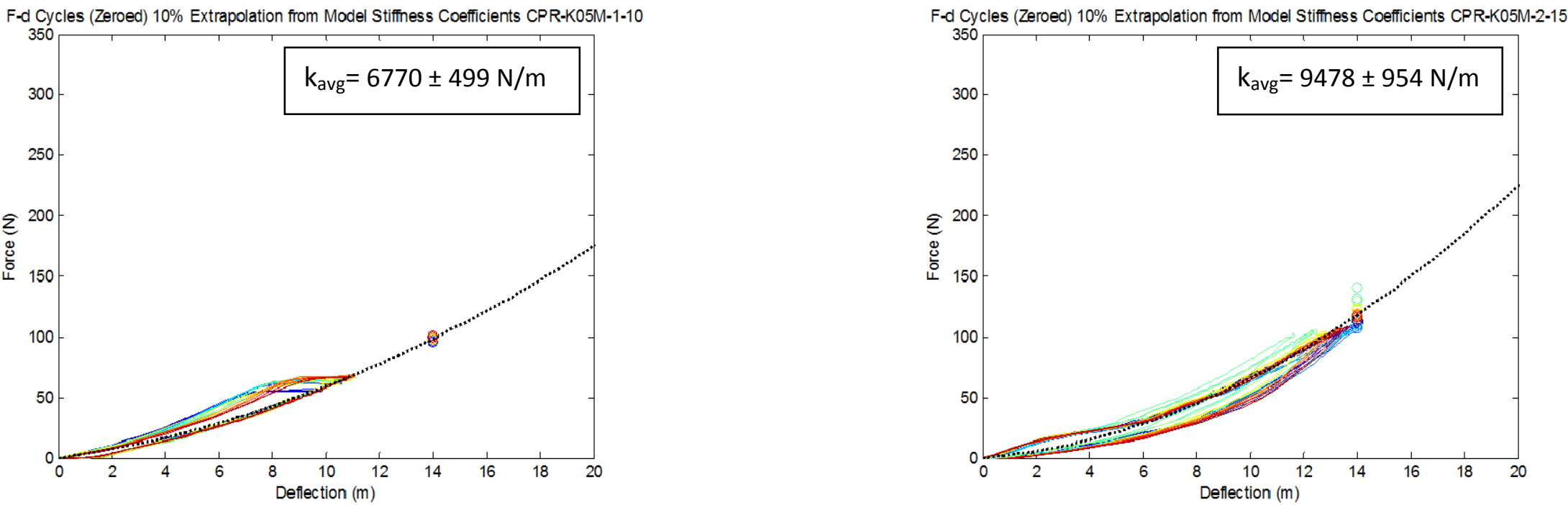

F-d Cycles (Zeroed) 10\% Extrapolation from Model Stiffness Coefficients CPR-K05M-3-10

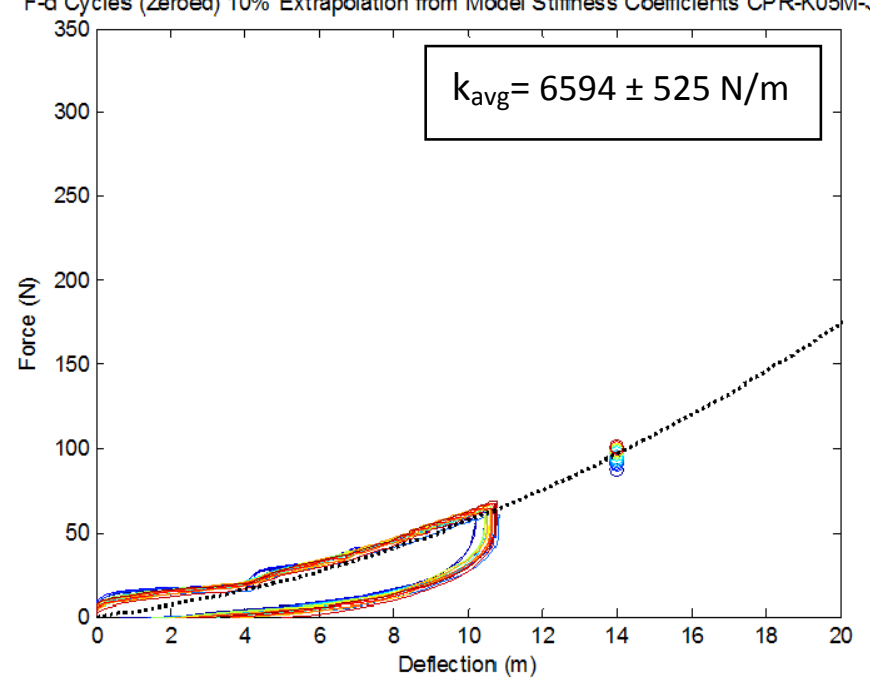




\section{Subject K06F}

F-d Cycles (Zeroed) 10\% Extrapolation from Model Stiffness Coefficients CPR-K06F-1-10

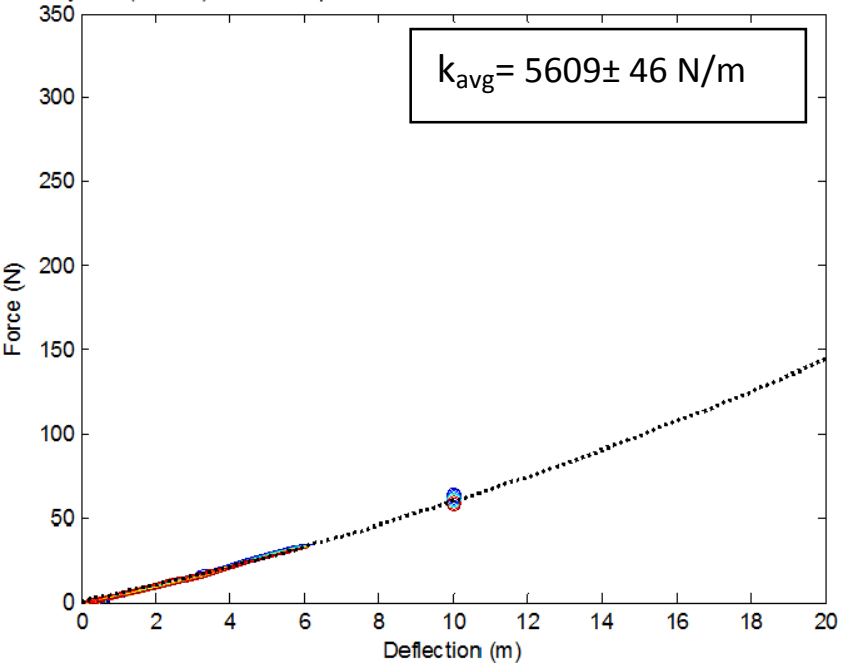

F-d Cycles (Zeroed) 10\% Extrapolation from Model Stiffness Coefficients CPR-K06F-3-10

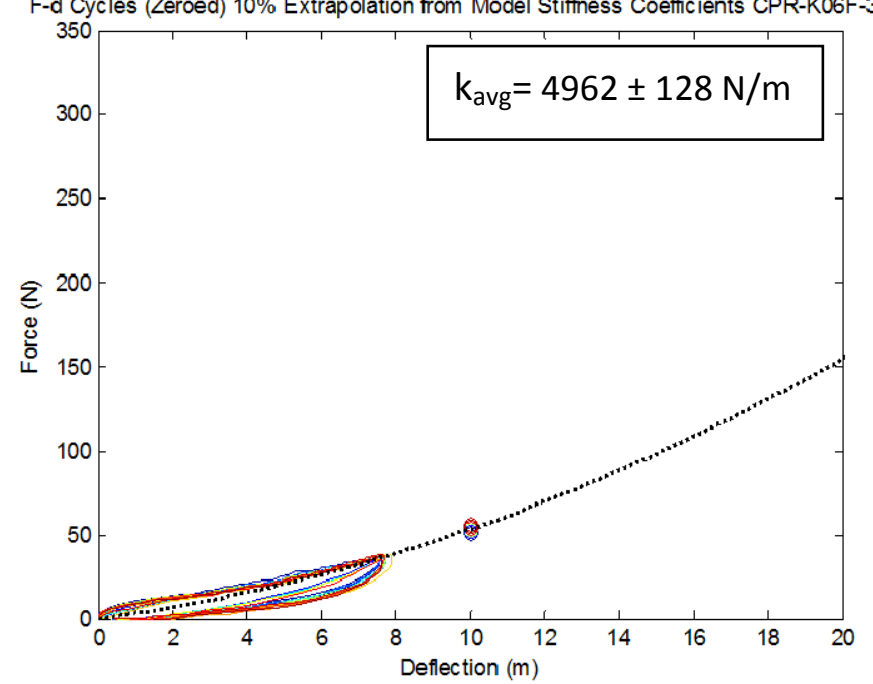

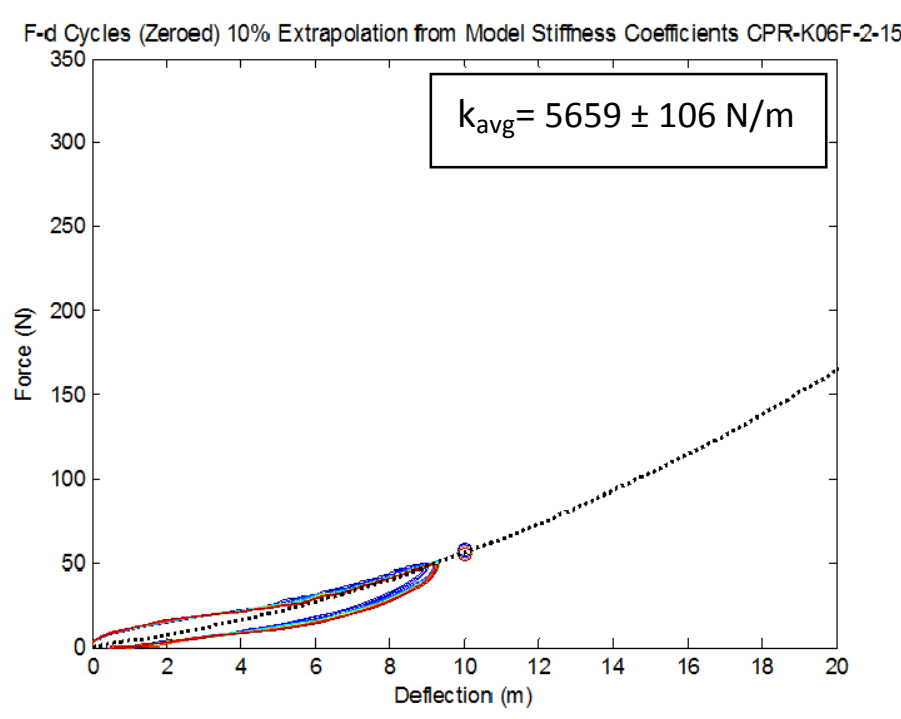




\section{Subject K08M}

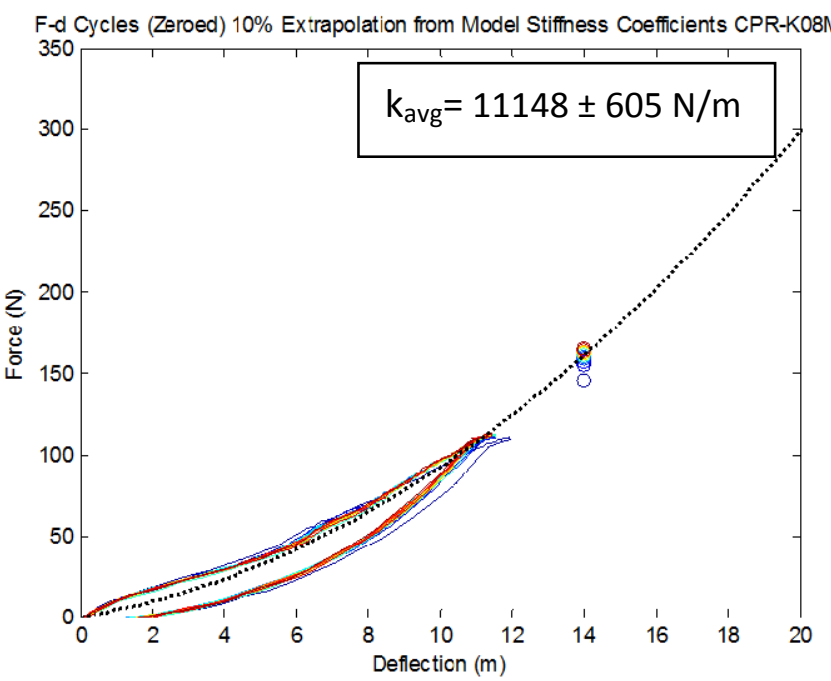

\section{Subject K10M}

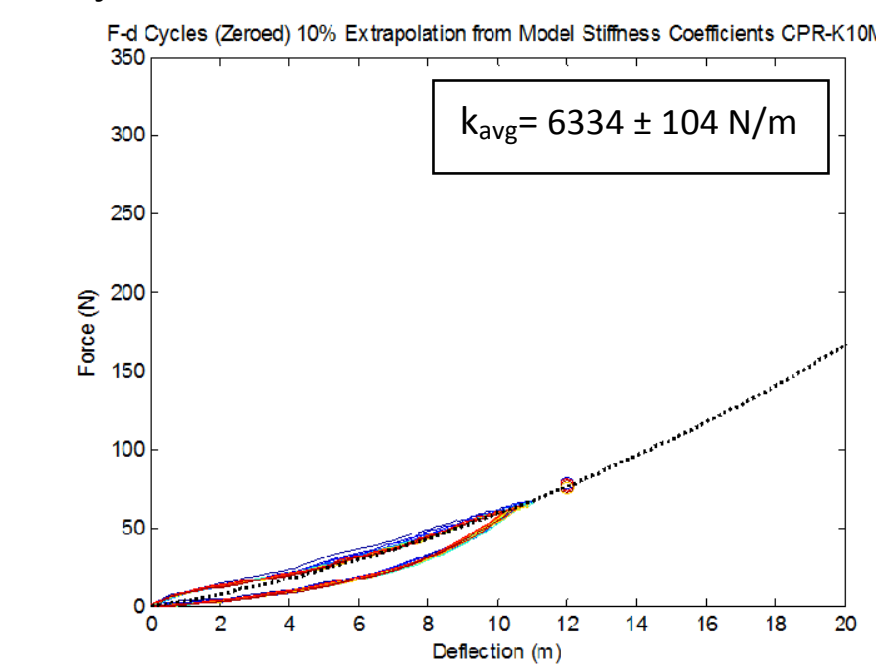

\section{Subject K09F}

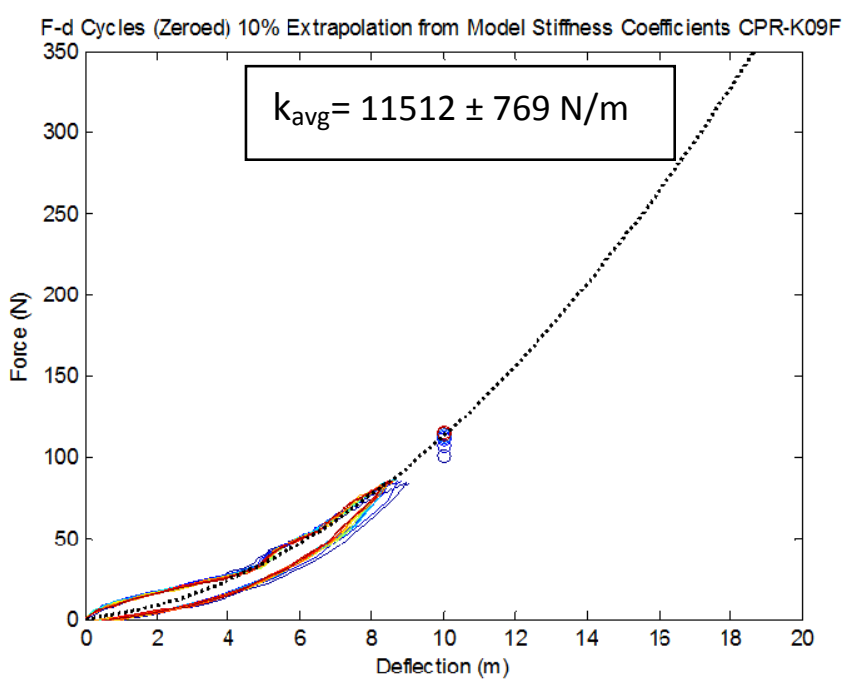




\section{Pediatric 6-year-old 484F}

F-d Cycles (Zeroed) 10\% Extrapolation from Model Stiffness Coefficients PEDVE021

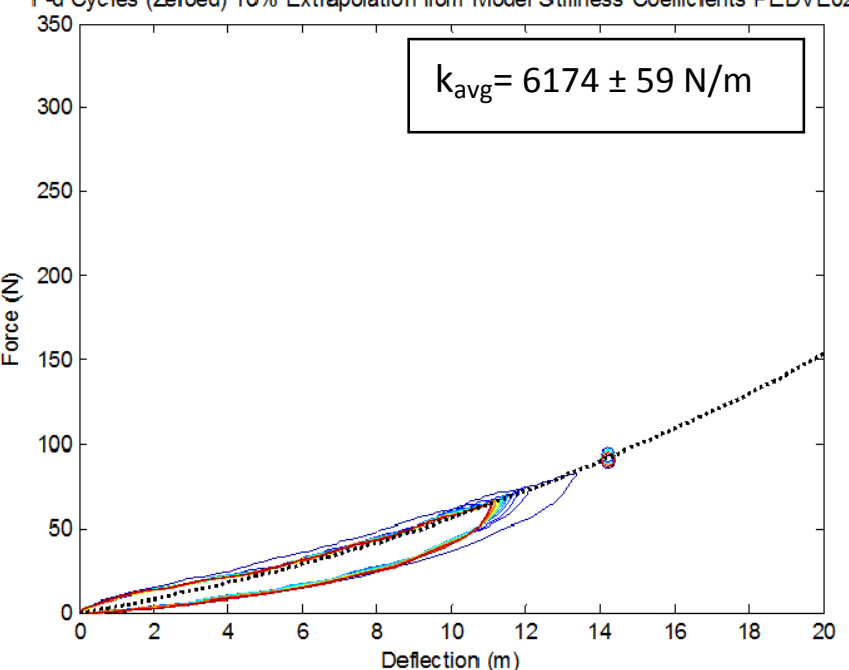

F-d Cycles (Zeroed) 10\% Extrapolation from Model Stiffiness Coefficients PEDVE023

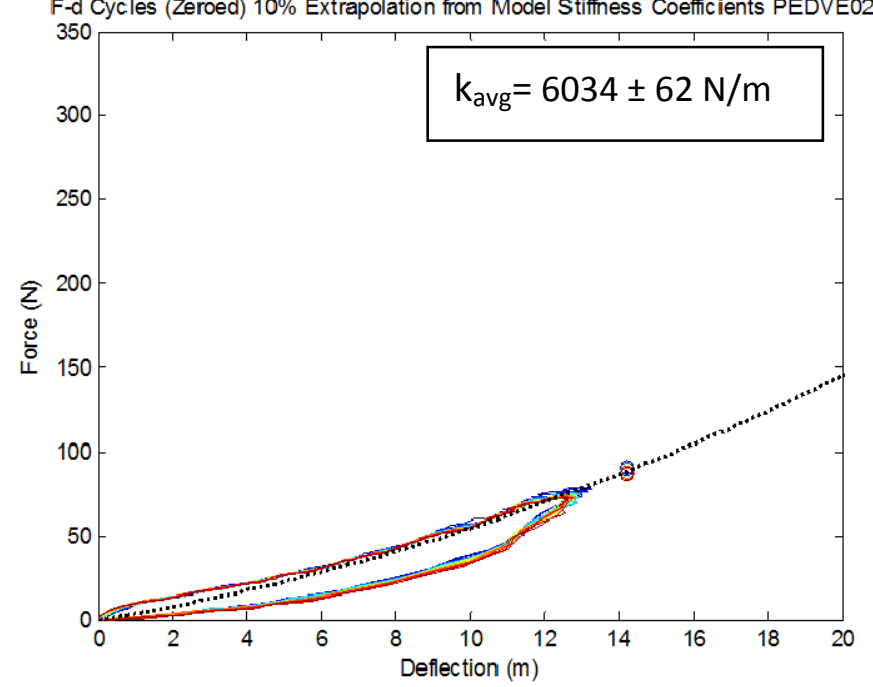

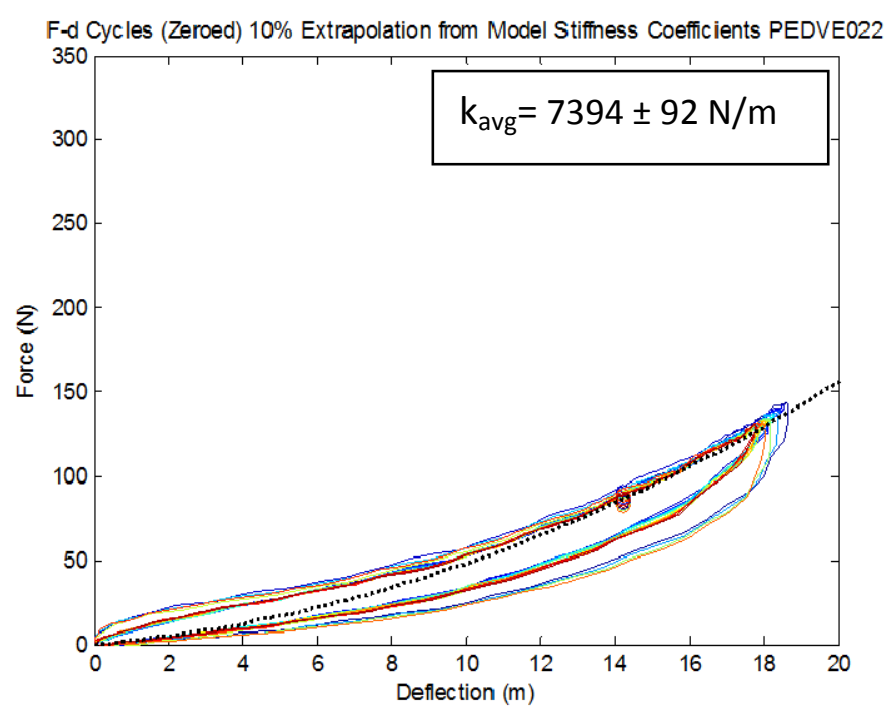




\section{Pediatric 7-year-old 470F}
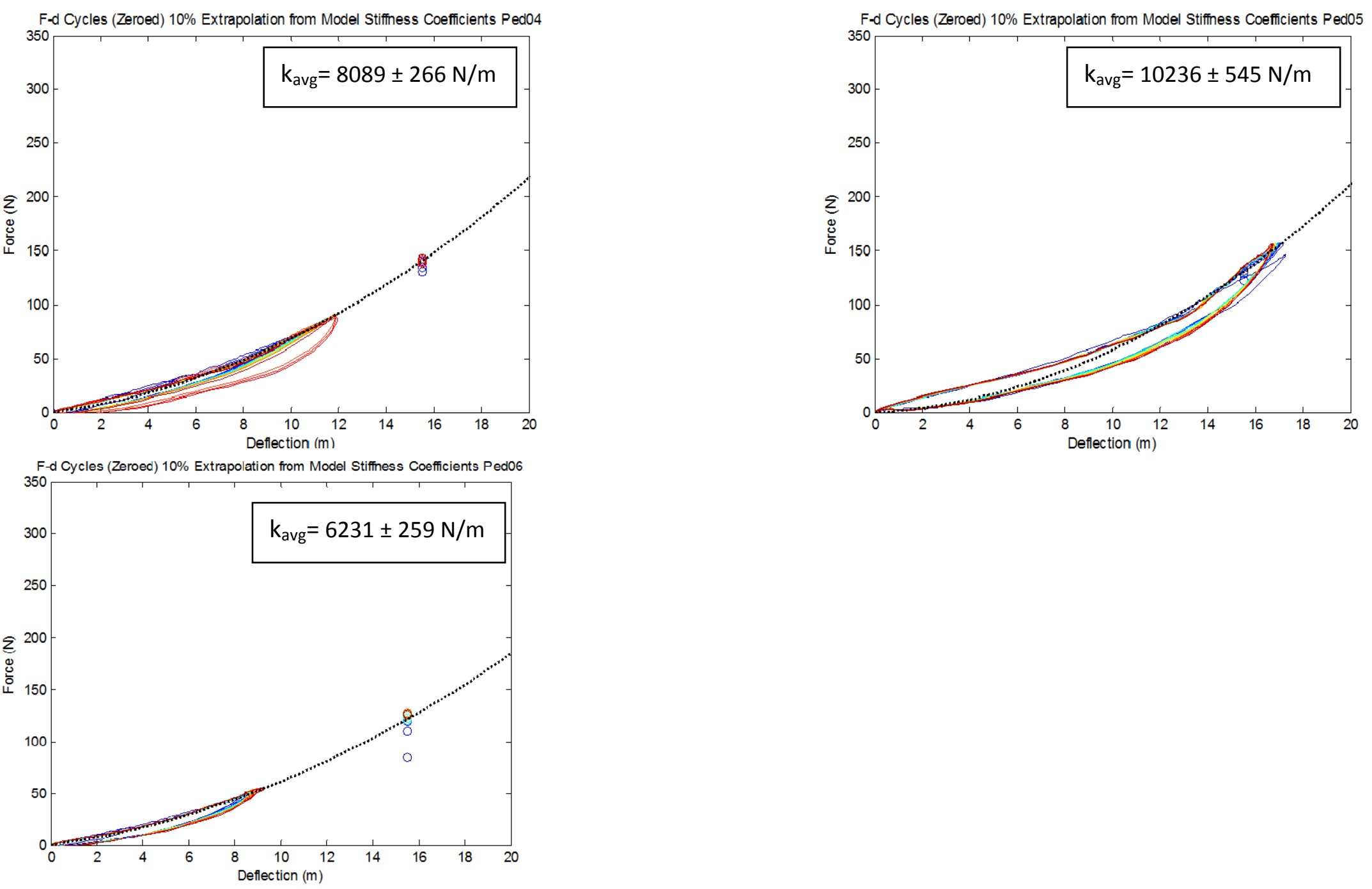
Table B1: Thoracic model elastic $\left(a_{1}\right.$ and $\left.a_{2}\right)$ and viscous $\left(b_{1}\right.$ and $\left.b_{2}\right)$ coefficients and linear stiffness (k_linear) for CPR tests on Pediatric Patients, Pediatric PMHS and Kangaroos (Maltese et al. 2008, Kent et al. 2012)

\begin{tabular}{|c|c|c|c|c|c|c|c|c|c|c|c|c|}
\hline \multirow[b]{2}{*}{ Test ID } & \multirow[b]{2}{*}{ Subject } & \multirow[b]{2}{*}{ Age (years) } & \multicolumn{2}{|c|}{$\mathrm{a} 1 \mathrm{~N} / \mathrm{m}$} & \multicolumn{2}{|c|}{$a 2 \mathrm{~N} / \mathrm{m}^{\wedge} 2$} & \multicolumn{2}{|c|}{ b1 Ns/m } & \multicolumn{2}{|c|}{ b2 Ns/m^^2 } & \multicolumn{2}{|c|}{ k_linear N/m } \\
\hline & & & Mean & SD & Mean & SD & Mean & SD & Mean & SD & Mean & SD \\
\hline 1 & Maltese 8yo & 8 & 3062 & 2237 & 122712 & 64809 & -5 & 80 & 8308 & 5938 & 7592 & 1151 \\
\hline 2 & Maltese 9yo & 9 & 1009 & 1141 & 99622 & 41511 & -27 & 51 & 7541 & 2753 & 5034 & 1059 \\
\hline Mean & & & 2036 & & 111167 & & -16 & & 7925 & & 6313 & \\
\hline SD & & & 1452 & & 16327 & & 16 & & 542 & & 1809 & \\
\hline Pedve021 & Kent 6yo & 6 & 3519 & 189 & 206928 & 14833 & 28 & 6 & 6519 & 743 & 5442 & 215 \\
\hline Pedve022 & Kent 6yo & 6 & 1818 & 373 & 299668 & 19343 & 19 & 17 & 6734 & 3221 & 7281 & 209 \\
\hline Pedve023 & Kent 6yo & 6 & 3584 & 41 & 184988 & 6091 & 35 & 3 & 3880 & 704 & 5657 & 126 \\
\hline Ped04 & Kent 7yo & 7 & 2681 & 406 & 414401 & 31192 & 52 & 5 & -1004 & 4120 & 6999 & 210 \\
\hline Ped05 & Kent 7yo & 7 & 977 & 499 & 480261 & 40735 & 47 & 10 & 3120 & 1089 & 8298 & 180 \\
\hline Ped06 & Kent 7yo & 7 & 2986 & 262 & 321214 & 33816 & 45 & 2 & 2315 & 578 & 5481 & 46 \\
\hline Mean & & & 2594 & & 317910 & & 38 & & 3594 & & 6527 & \\
\hline SD & & & 1021 & & 114889 & & 13 & & 2881 & & 1179 & \\
\hline K01F & K01 & $\mathrm{n} / \mathrm{a}$ & 3161 & 234 & 108645 & 28635 & 42 & 10 & -2311 & 1035 & 3077 & 270 \\
\hline K02F_1_10 & K02 & $\mathrm{n} / \mathrm{a}$ & 2232 & 258 & 377764 & 66980 & 40 & 12 & -3988 & 565 & 4013 & 323 \\
\hline K02F_2_15 & K02 & $\mathrm{n} / \mathrm{a}$ & 1592 & 201 & 525446 & 24716 & 35 & 2 & 5324 & 1330 & 6771 & 306 \\
\hline K02F_3_10 & K02 & $\mathrm{n} / \mathrm{a}$ & 2892 & 145 & 408373 & 20290 & 36 & 3 & 15094 & 1469 & 6198 & 137 \\
\hline K05M_1_10 & K05 & $\mathrm{n} / \mathrm{a}$ & 3041 & 252 & 284824 & 23819 & -9 & 8 & -7044 & 647 & 5050 & 320 \\
\hline K05M_2_15 & K05 & $\mathrm{n} / \mathrm{a}$ & 1863 & 397 & 469668 & 40067 & 31 & 4 & 3607 & 690 & 6768 & 503 \\
\hline K05M_3_10 & K05 & $\mathrm{n} / \mathrm{a}$ & 2629 & 569 & 304721 & 55585 & 31 & 4 & 18103 & 1487 & 5181 & 169 \\
\hline K06F_1_10 & K06 & $\mathrm{n} / \mathrm{a}$ & 4732 & 114 & 124183 & 26911 & 20 & 3 & -4413 & 639 & 4725 & 369 \\
\hline K06F_2_15 & K06 & $\mathrm{n} / \mathrm{a}$ & 2899 & 253 & 266757 & 17646 & 46 & 2 & 7041 & 489 & 4883 & 320 \\
\hline K06F_3_10 & K06 & $\mathrm{n} / \mathrm{a}$ & 2922 & 430 & 241827 & 52408 & 48 & 17 & 7756 & 6478 & 4580 & 149 \\
\hline K08M & K08 & $\mathrm{n} / \mathrm{a}$ & 3477 & 432 & 573051 & 52069 & 60 & 4 & 3003 & 512 & 8448 & 381 \\
\hline K09F & K09 & $\mathrm{n} / \mathrm{a}$ & 2643 & 475 & 866482 & 78450 & 35 & 2 & 5696 & 1036 & 8355 & 516 \\
\hline K10M & K10 & $\mathrm{n} / \mathrm{a}$ & 3455 & 386 & 242527 & 31543 & 25 & 2 & 4865 & 635 & 5453 & 352 \\
\hline Mean & & & 2888 & & 368790 & & 34 & & 4056 & & 5654 & \\
\hline SD & & & 788 & & 205532 & & 16 & & 7350 & & 1593 & \\
\hline
\end{tabular}




\section{Appendix C: Bone Coordinate System}

Location and orientation for vertebral body (T4 example) and the pelvis
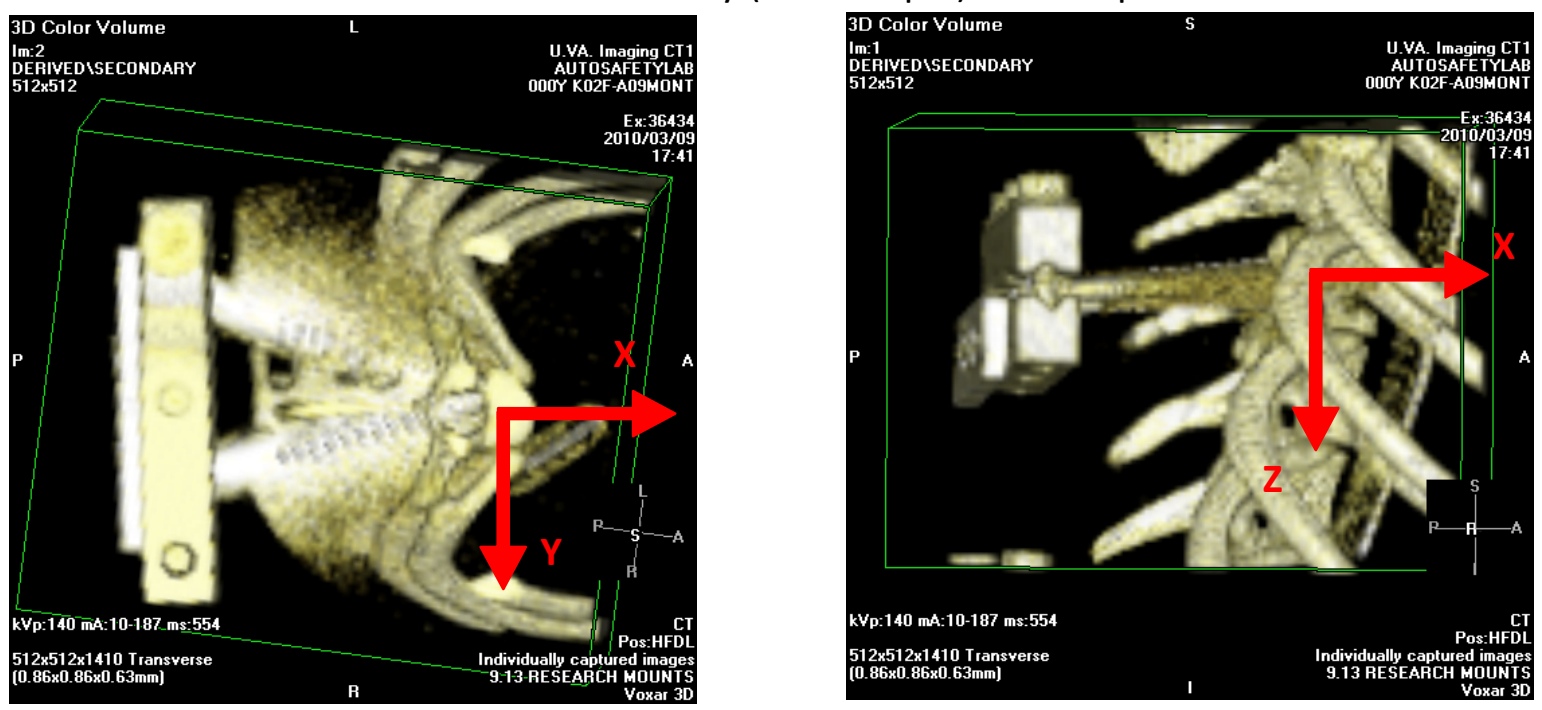

T4
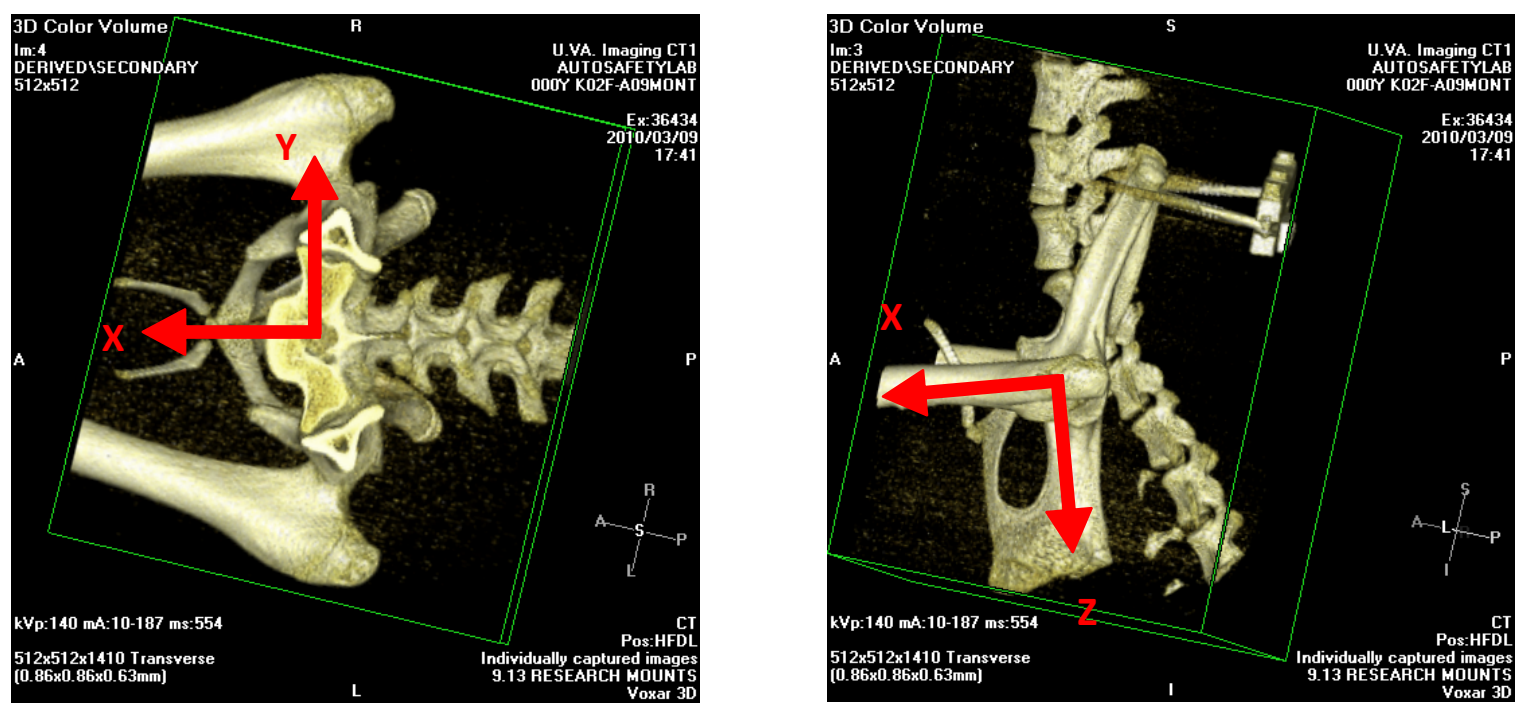

Pelvis 


\section{Appendix D: Sled Test Subject Positioning Information}

\section{Kangaroo subject positioning measurements for sled tests}

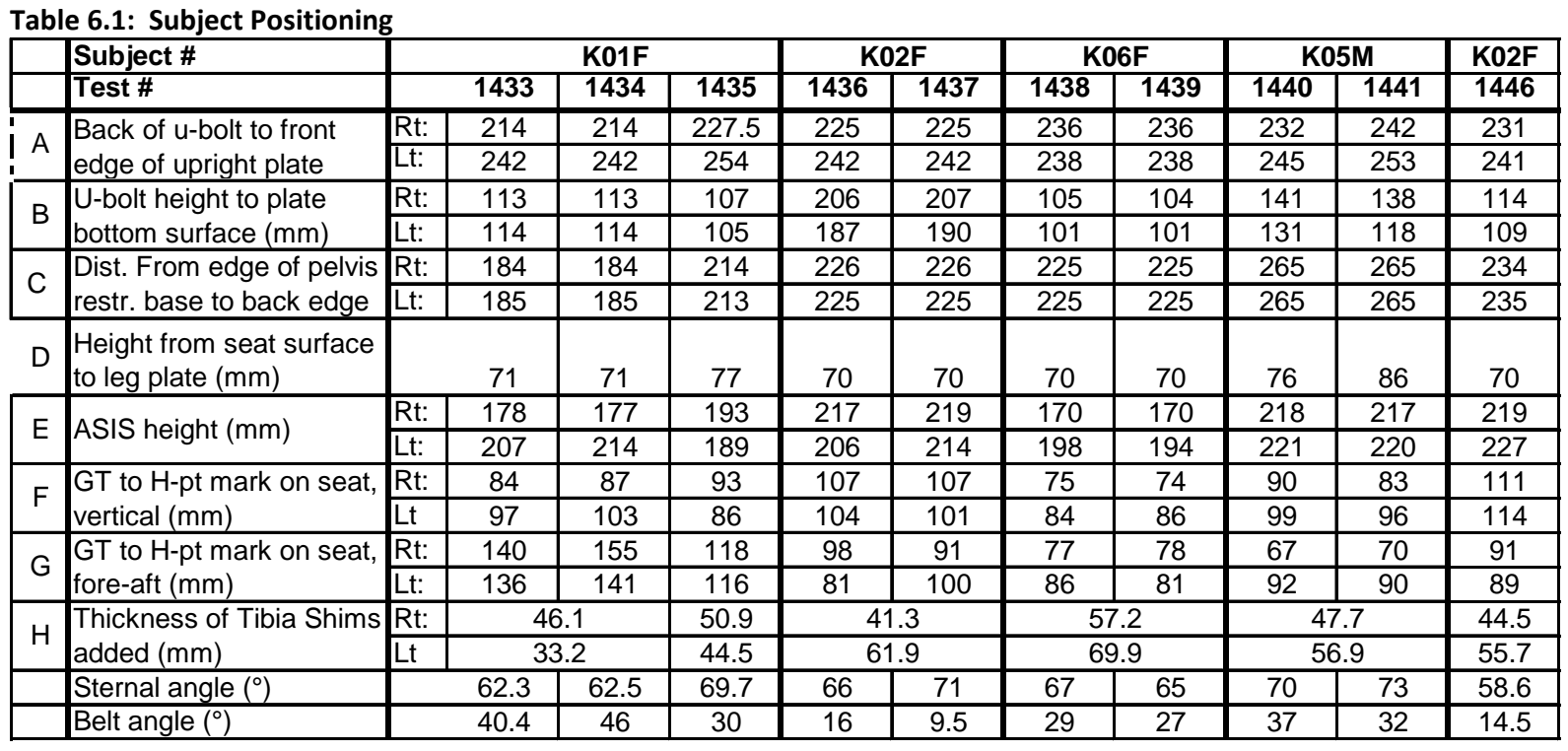

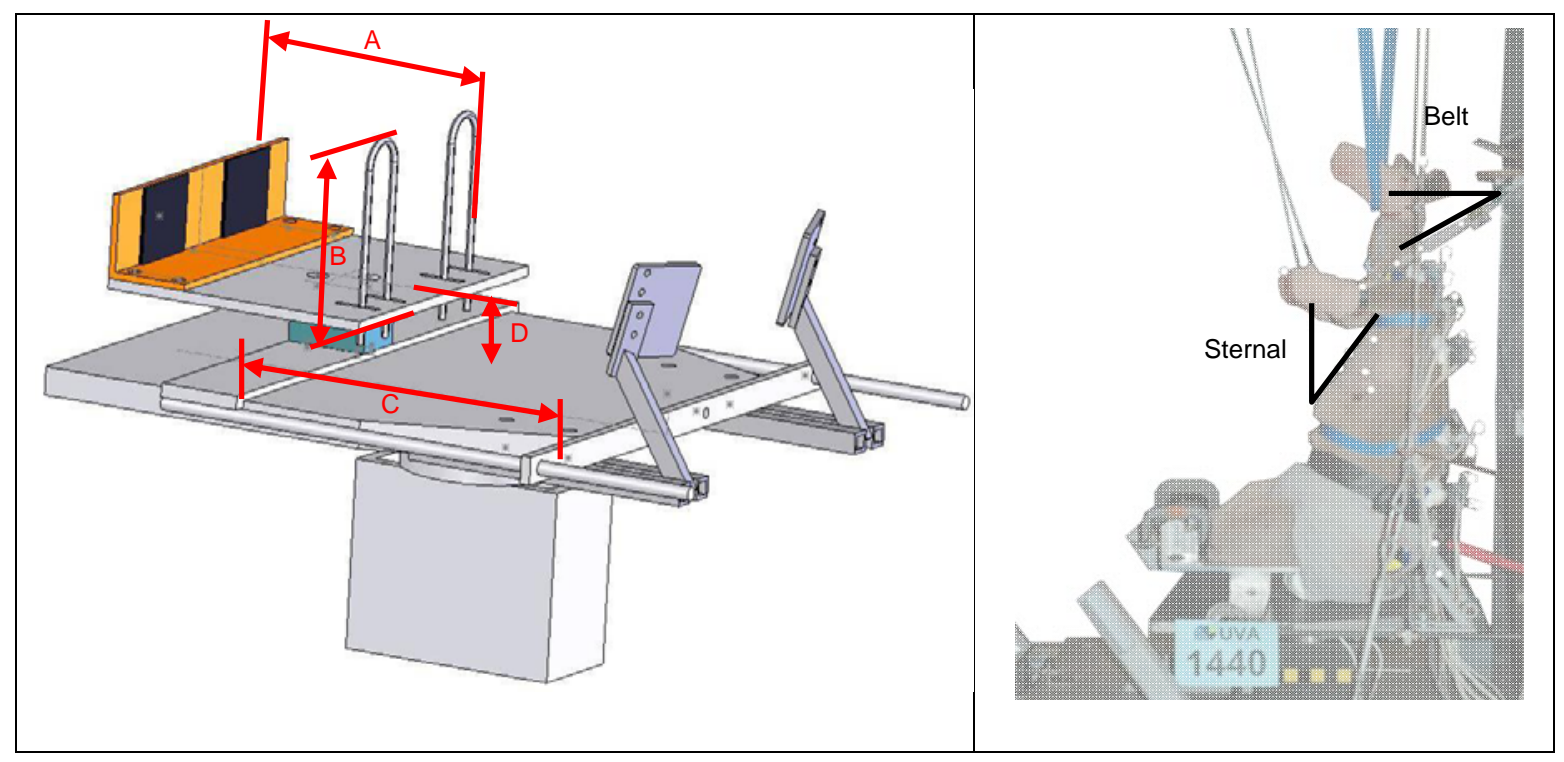




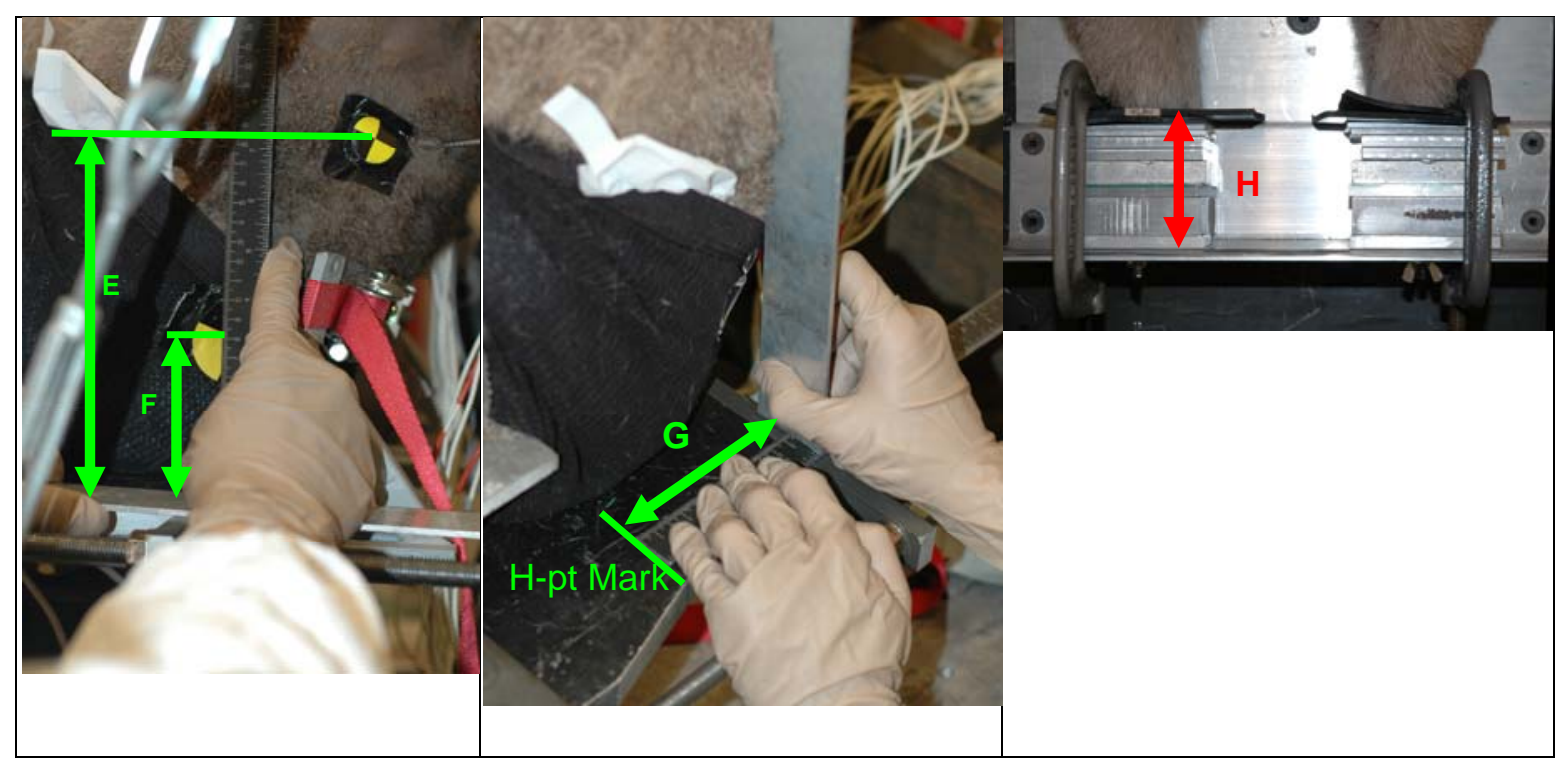

Table 6.2: Additional Positioning Measurements

\begin{tabular}{|c|c|c|c|c|c|c|c|c|c|c|c|}
\hline & & \multicolumn{2}{|c|}{ Arm Suspension } & \multicolumn{2}{|c|}{ Head support bolt } & \multirow{2}{*}{\begin{tabular}{|c|} 
Head Tether \\
length
\end{tabular}} & \multicolumn{4}{|c|}{ Cross Bars Height Positioning } & \multirow{2}{*}{$\frac{\text { D-ring }}{C}$} \\
\hline & & Right & Left & lateral & fore-aft & & A left & A right & B left & B right & \\
\hline Subject & Test \# & $\mathrm{mm}$ & $\mathrm{mm}$ & $\mathrm{mm}$ & $\mathrm{mm}$ & $\mathrm{mm}$ & $\mathrm{mm}$ & $\mathrm{mm}$ & $\mathrm{mm}$ & $\mathrm{mm}$ & $\mathrm{mm}$ \\
\hline \multirow{3}{*}{ K01F } & 1433 & $\mathrm{n} / \mathrm{a}$ & $\mathrm{n} / \mathrm{a}$ & \multirow{3}{*}{515} & \multirow{3}{*}{117} & 1450 & \multirow{3}{*}{1140} & \multirow{3}{*}{1135} & \multirow{3}{*}{1005} & \multirow{3}{*}{1021} & 403 \\
\hline & 1434 & $\mathrm{n} / \mathrm{a}$ & $\mathrm{n} / \mathrm{a}$ & & & 1450 & & & & & 454 \\
\hline & 1435 & 720 & 720 & & & 1250 & & & & & 454 \\
\hline \multirow{2}{*}{$\mathrm{K} 02 \mathrm{~F}$} & 1436 & \multirow{2}{*}{570} & \multirow{2}{*}{550} & \multirow{2}{*}{515} & \multirow{2}{*}{155} & 950 & \multirow{2}{*}{1202} & \multirow{2}{*}{1195} & \multirow{2}{*}{1012} & \multirow{2}{*}{1020} & \multirow{2}{*}{456} \\
\hline & 1437 & & & & & 1140 & & & & & \\
\hline \multirow{2}{*}{ K06F } & 1438 & \multirow{2}{*}{710} & \multirow{2}{*}{685} & \multirow{2}{*}{515} & \multirow{2}{*}{155} & \multirow{2}{*}{1050} & \multirow{2}{*}{115} & \multirow{2}{*}{114} & \multirow{2}{*}{95} & \multirow{2}{*}{93} & \multirow{2}{*}{455} \\
\hline & 1439 & & & & & & & & & & \\
\hline \multirow{2}{*}{ K05M } & 1440 & \multirow{2}{*}{635} & \multirow{2}{*}{623} & \multirow{2}{*}{$\mathrm{n} / \mathrm{a}$} & $\mathrm{n} / \mathrm{a}$ & $\mathrm{n} / \mathrm{a}$ & $\mathrm{n} / \mathrm{a}$ & $\mathrm{n} / \mathrm{a}$ & 1080 & 1075 & 465 \\
\hline & 1441 & & & & & & & & & & \\
\hline $\mathrm{K} 02 \mathrm{~F}$ & 1446 & 595 & 570 & 515 & 152 & 935 & 1197 & 1200 & 1024 & 1025 & 465 \\
\hline
\end{tabular}

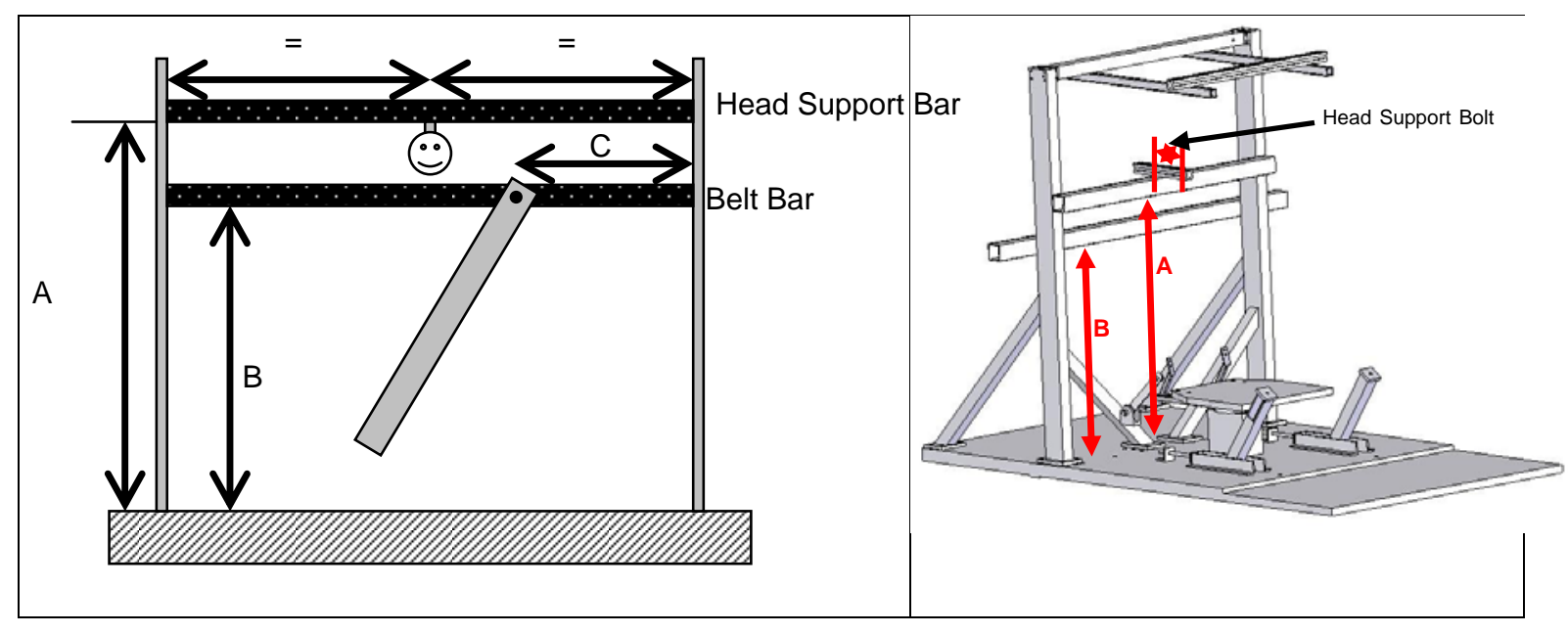




\section{Appendix E: Kangaroo Sled Test Force Deflection Data}

Upper shoulder belt tension and chest deflection curves for kangaroo low and high speed tests. Chest deflection was obtained from Vicon motion capture data which was noisy.
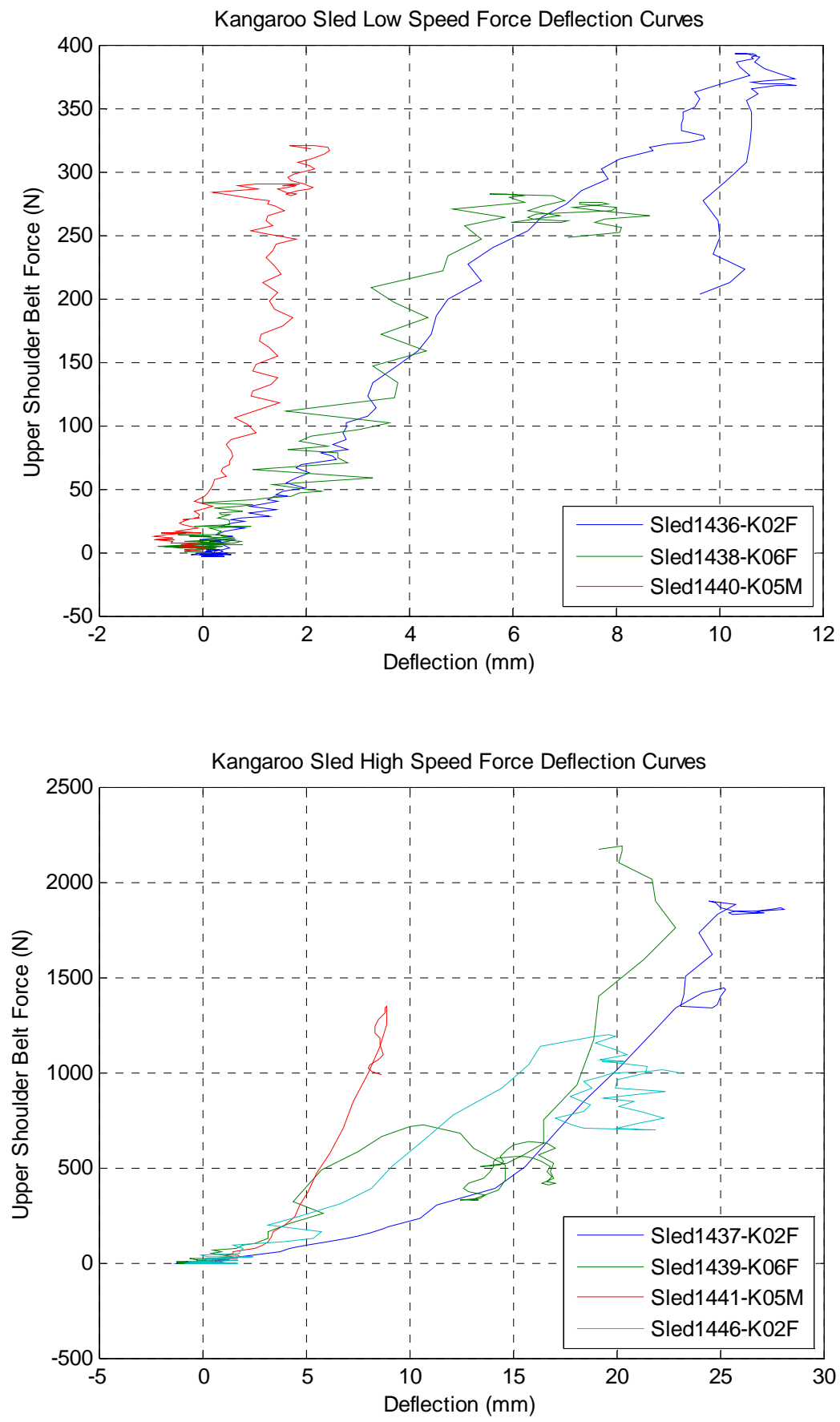